\title{
Experimental investigation of Cloud droplet dynamics at the research station Schneefernerhaus
}

\author{
Dissertation \\ for the award of the degree \\ Doctor rerum naturalium (Dr.rer.nat.) \\ of the Georg-August-Universität Göttingen \\ within the doctoral program GGNB \\ of the Georg-August University School of Science (GAUSS) \\ Submitted by \\ Augustinus Cornelis Maria Bertens \\ from Boxtel, The Netherlands
}

Göttingen, 2021 
Thesis committee

Dr. Jan Moláček

LFPN, MPI for Dynamics and Self-Organisation

Prof. Andreas Dillmann

Institut of Aerodynamics and Flow Technology, DLR

Prof. Jens Niemeyer

Institute of Astrophysics, Georg-August-Universität Göttingen

Members of the Examination Board

First referee: Dr. Jan Moláček

LFPN, MPI for Dynamics and Self-Organisation

Second referee: Prof. Andreas Dillmann

Institut of Aerodynamics and Flow Technology, DLR

Further members of the Examination Board

Dr. Michael Wilczek

Turbulence, Complex Flows \& Active Matter, MPI for Dynamics and SelfOrganisation

Prof. Andreas Tilgner

Institute of Geophysics, Georg-August-Universität Göttingen

Prof. Annette Zippelius

Institute for Theoretical Physics, Georg-August-Universität Göttingen

Date of oral examination: 17-6-2021.

Revision: 5cc7777 


\section{Abstract}

The collision-coalescence of droplets in turbulence is responsible for the fast growth of cloud droplets from $15 \mu \mathrm{m}$ to $40 \mu \mathrm{m}$ in radius, but how exactly it causes this quick growth is not understood. The growth of cloud droplets through collision-coalescence is governed by two quantities: the radial distribution function (RDF), which is a measure for the degree of clustering the droplets exhibit, and the radial relative velocity (RRV), which is a measure for the velocity difference between nearby droplets.

In this thesis an in-situ experiment is described, that is designed to simultaneously measure all aspects relevant to turbulent collision-coalescence of cloud droplets: droplet motions, droplet sizes, and properties of the turbulent carrier flow. The experiment is located in the German Alps, on top of the environmental research station Schneefernerhaus, at an altitude of $2650 \mathrm{~m}$ where clouds naturally occur. Droplet motions are measured using a particle tracking setup; turbulence statistics are measured with a sonic anemometer that is mounted close by.

Droplet sizes are measured using a novel technique that relies only on the droplet intensities as recorded by the particle tracking experiment. A complete derivation of the technique based on Lorentz-Mie scattering theory is given. Droplet sizes measured with this approach are compared to those obtained with a holographic instrument.

The experiment is used to measure droplet rms accelerations and the radial distribution function conditioned on (pairs of) Stokes numbers. Both qualitatively agree with literature values, but further research is needed to see why there is no quantitative agreement. 


\section{Contents}

$\begin{array}{lll}1 & \text { Introduction } & 9\end{array}$

\begin{tabular}{|ll|l}
2 & Theory 13 & 13
\end{tabular}

2.1 Turbulence . . . . . . . . . . . . . . . . . . . . . . 13

2.1 .1 Reynolds equations. . . . . . . . . . . . . . . 15

2.1 .2 Structure functions $\ldots \ldots \ldots \ldots$. . . . . . . . . . 16

2.1 .3 Two-point velocity autocorrelation . . . . . . . . . . . . 18

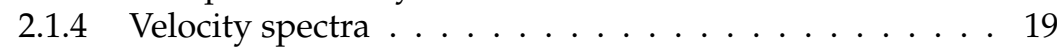

2.2 Droplet kinematics . . . . . . . . . . . . . . . . . . . . . . 21

2.3 Cloud droplet growth . . . . . . . . . . . . . . . . . . 23

2.3 .1 The collision kernel . . . . . . . . . . . . . . . . . . 26

\begin{tabular}{ll|l}
3 & Setup & 29 \\
& 3.1 &
\end{tabular}

3.1 Location . . . . . . . . . . . . . . . . . . . . . . . . . . . 29

3.2 Seesaw and vibration damping . . . . . . . . . . . . 32

3.3 Control and data acquisition $\ldots \ldots \ldots \ldots$

3.4 Other instruments . . . . . . . . . . . . . . . . . . . . 34

4 Particle tracking 35

4.1 Setup . . . . . . . . . . . . . . . . . . . 35

4.1 .1 Camera Box . . . . . . . . . . . . . . . . 35

4.1 .2 Optics $\ldots \ldots \ldots \ldots \ldots \ldots$

4.1 .3 Illumination . . . . . . . . . . . . . . . . . . . . . . . . 39

4.1 .4 Camera positions and viewing angles . . . . . . . . . . 40

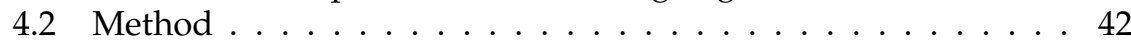

4.2 .1 Start of season . . . . . . . . . . . . . . . . . . 42

4.2 .2 Calibration. . . . . . . . . . . . . . . . 43

4.2 .3 Single experiment $\ldots \ldots \ldots \ldots$. . . . . . . . 43 
4.3 Data analysis . . . . . . . . . . . . . . . . . . . . . . 44

4.4 Verification . . . . . . . . . . . . . . . . . . . . . . . . 46

4.4 .1 Kinematic simulation . . . . . . . . . . . . . . 46

5 Droplet sizing 51

5.1 Introduction $\ldots \ldots \ldots \ldots \ldots \ldots \ldots \ldots$

5.2 Techniques . . . . . . . . . . . . . . . . . . . . 51

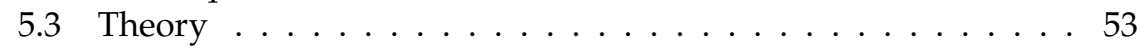

5.3 .1 Lorentz-Mie scattering . . . . . . . . . . . . . . . . 53

5.3 .2 Integration of scattered intensity . . . . . . . . . . 57

5.4 Method . . . . . . . . . . . . . . . . . . . 59

5.5 Calibration . . . . . . . . . . . . . . . . . . . . . 65

5.6 Results $\ldots \ldots \ldots \ldots \ldots \ldots 6 \ldots$

5.7 Conclusion . . . . . . . . . . . . . . . . . . . . . . . . . . . . . . 69

5.8 Outlook . . . . . . . . . . . . . . . . . . . . . . . . . . . 69

$\begin{array}{lll}6 & \text { Turbulence statistics } & \mathbf{7 1}\end{array}$

6.1 Setup . . . . . . . . . . . . . . . . . 72

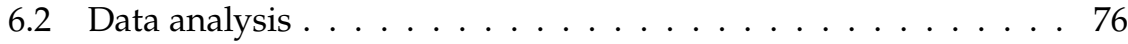

6.2 .1 Indexing . . . . . . . . . . . . . . . . 77

6.2 .2 Preprocessing . . . . . . . . . . . . . . . . . 78

6.2 .3 Segmentation . . . . . . . . . . . . . . . . . . . . . . . . 79

6.2 .4 Binning. . . . . . . . . . . . . . . . . . . . . . . . . 80

6.2 .5 Computing turbulence statistics $\ldots \ldots \ldots$. . . . . . 80

$6.2 .6 \quad$ Creating daily graphs $\ldots \ldots \ldots \ldots$. . . . . . . . . . 82

6.3 Results ......................... . . 82

$6.3 .1 \quad$ Segmentation and binning. . . . . . . . . . . . . 82

6.3 .2 Per-bin statistics . . . . . . . . . . . . . . . . . . . . . . . . 82

6.3 .3 Daily graphs . . . . . . . . . . . . . . . . . . 84

6.4 Conclusion $\ldots \ldots \ldots \ldots \ldots \ldots \ldots$

$\begin{array}{lll}7 & \text { Droplet dynamics } & 89\end{array}$

7.1 Accelerations $\ldots \ldots \ldots \ldots$. . . . . . . . . . . . . 90

7.1 .1 Theory . . . . . . . . . . . . . . . . . . 90 90

7.1 .2 Method . . . . . . . . . . . . . . . . . . . . . . . . 991

7.1 .3 Results $\ldots \ldots \ldots \ldots \ldots 1$

7.2 Radial distribution function $\ldots \ldots \ldots \ldots$. . . . . . . . . 94

7.2 .1 Theory . . . . . . . . . . . . . . . . . . . 994

7.2 .2 Method . . . . . . . . . . . . . . . . . . . . . 96

$7.2 .3 \quad$ Results $\ldots \ldots \ldots \ldots$. . . . . . . . . . . . 97 
7.3 Conclusion . . . . . . . . . . . . . . . . 100

\begin{tabular}{lll}
\hline 8 & Conclusion & 101
\end{tabular}

\begin{tabular}{|ll}
\hline A Integration of scattered irradiance & 105
\end{tabular}

\begin{tabular}{lll}
\hline B Bayesian inference of droplet size & 109
\end{tabular}

B.1 Bayesian inference . . . . . . . . . . . . . . . . . . . . . 109

B.2 Bayesian droplet sizing . . . . . . . . . . . . . . . . . . . . . . . . 110

B.3 Model parameters . . . . . . . . . . . . . . . . . . . . 111

B.3.1 Estimate from histograms . . . . . . . . . . . . . . . 111

B.3.2 Maximum likelihood estimation . . . . . . . . . . . . 112

B.4 Droplet sizing . . . . . . . . . . . . . . . . 116

B.5 Conclusion . . . . . . . . . . . . . . . . 116

\begin{tabular}{|l|l|}
\hline Calibration of the FMAG aerosol generator & 121
\end{tabular}

C.1 The FMAG aerosol generator . . . . . . . . . . . . . . . . . . . . . . . . . . . 121

C.2 Shadowgraphy. . . . . . . . . . . . . . . . . . . 122

C.2.1 Setup . . . . . . . . . . . . . . . . 122

C.2.2 Data analysis . . . . . . . . . . . . . . . . . . . . . . . . . . . . . . . . . . . . . . . . .

C.3 Results . . . . . . . . . . . . . . . . . . . . . 125

C.4 Notes . . . . . . . . . . . . . . . . . . . . . 127

\begin{tabular}{ll|}
\hline D Mean-wind compensation & 129
\end{tabular}

D.1 Seesaw . . . . . . . . . . . . . . . . . . . 130

D.2 Vibration damping . . . . . . . . . . . . . . . . . . . . . . . . . . . . . . . . . . . . . . . . . . . . . .

D.2.1 Table vibration . . . . . . . . . . . . . . . 132

D.2.2 Passive damping . . . . . . . . . . . . . . . . . . 133

D.2.3 Active damping . . . . . . . . . . . . . . . . . . . . . . . . . . . . . . . . . . . . . . . . . . . . .

D.3 Conclusion . . . . . . . . . . . . . . . . . . . 142

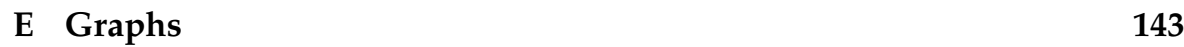

E.1 Turbulent statistics . . . . . . . . . . . . . . . . . . . . . . . . . 143

E.2 Droplet accelerations . . . . . . . . . . . . . . . . . . . . . 147

E.3 Droplet acceleration fits . . . . . . . . . . . . . . . . . . . . 148

E.4 Clustering exponent fits . . . . . . . . . . . . . . . 150

\begin{tabular}{ll}
\hline Bibliography & 153
\end{tabular}

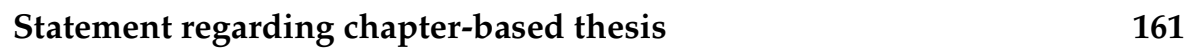


CONTENTS

Curriculum Vitae 163 


\section{Chapter 1}

\section{Introduction}

This thesis is concerned with rain formation. Rain forms in four phases: activation, condensation, turbulent collision-coalescence, and finally gravitational collision-coalescence. Each of these phases acts on droplets of a certain size. In particular condensation is effective for droplet radii $\lesssim 15 \mu \mathrm{m}$, whereas gravitational collision-coalescence is effective for radii $\gtrsim 40 \mu \mathrm{m}$. The size gap in between these two phases is bridged by turbulent collision-coalescence; this phase is responsible for colliding small droplets with each other, causing them to coalesce and quickly grow in size [14, 24].

How exactly turbulence causes this quick growth of droplets, is not understood. Two popular theories are preferential concentration, and the slingshot effect. Preferential concentration means that droplets prefer to concentrate in certain areas in a turbulent flow [5]. This causes the local droplet density to be higher than the average, which could increase the collision rate. However, droplet velocities in this case are correlated to that of the underlaying turbulent flow, and hence to each other, which would prevent collisions. Furthermore, as a result of preferential concentration, they also preferentially sample certain parts of the flow, which, in turn, biases their dynamics.

The slingshot effect describes droplets that are slung from one region of the flow into another. The velocity of such a droplet is not correlated to that of the underlaying flow, and therefore will be dissimilar from the velocities of droplets in its immediate surroundings. As a result the chance that it collides with another droplet can increase, which in turn could, again, increase the collision rate [16].

The idea that differential velocities could increase the collision rate was studied by Saffman and Turner [55]. Using basic knowledge from turbulence 
theory and simple geometry, they derived an expression for the collision rate. Their collision rates depend heavily on the two droplet sizes under consideration (assuming binary collisions) and the turbulent dissipation rate, a measure for the intensity of the turbulence. Indeed they show that as the turbulent dissipation rate increases, the collision rate also increases.

To compute the collision rate two ingredients are needed: (1) the chance that two droplets can be found at near-touching range; and (2) the relative velocity of these droplets. The first of these is given by the radial distribution function, or RDF, which measures the relative probability to find a droplet at certain distance from another droplet. The second is given by the radial relative velocity, or RRV.

The goal of studying turbulent collision-coalescence is twofold: most importantly, improving our understanding of this process in clouds, helps us to improve weather and climate forecasting. Collision-coalescence is not resolved in weather models, and is modelled instead. Such microphysical processes are a source of uncertainty for weather models [24]. Better data, whether they come from idealised direct numerical simulations (DNS) or experiments, can be used to improve these models [14].

It is difficult to study cloud droplet growth numerically. Direct numerical simulations (DNS) cannot be performed at Reynolds numbers that are representative of cloud turbulence. They are nevertheless useful tools to study smaller, idealised systems, because in such simulations the entire velocity field is known exactly, and these therefore allow for very elaborate studies [14]. Much larger Reynolds number flows can be simulated using large-eddy simulations (LES). However, in LES only the large scales are resolved, while the smaller scales are modelled [48, ch. 13], and as a result it not possible to study the effects of the smaller scales on the droplets.

It is equally difficult to study cloud droplet growth using laboratory experiments, because they cannot correctly reproduce all of the parameters relevant to collision-coalescence. To reach sufficiently high Reynolds numbers, either a very large experiment must be constructed, which is prohibitively expensive, or the dissipation rate must be increased. The result of that, however, is that the Froude number $\operatorname{Fr}_{f}=\left(v / \eta^{3}\right)^{1 / 4} / g$, which is a non-dimensional measure for the importance of gravity, changes. Furthermore, the Kolmogorov length scale $\eta=\left(v^{3} / \epsilon\right)^{1 / 4}$ decreases, which may make it difficult to observe processes occuring at this scale. A good example of this are the experiments done in the Max-Planck Variable Density Turbulence Tunnel in Göttingen [8], in which Taylor Reynolds numbers of up to 5000 are reached. At such high Reynolds numbers the Kolmogorov length scale $\eta$ is only $9 \mu \mathrm{m}$, which is 3 to 5 times shorter than the hot-wire probes used to measure the flow [34]. 
At present the only way to study cloud droplet growth in representative conditions, is by means of in-situ experiments. These experiments typically make use of aircraft or aerostats to get the instruments into the clouds. While such experiments have the freedom to choose both cloud type (marine vs. continental) and cloud region (core vs. boundary), they are constrained by either spatial resolution (in case of aircraft) or by weight (in case of aerostats). The HALOHolo, a holographic instrument that is designed to be carried on aircraft, records up to 6 holograms per second. If it is carried by an aircraft travelling at $40 \mathrm{~m} \mathrm{~s}^{-1}$, it acquires one hologram every 6 metres. An example of an aerostat is the The Max-Planck CloudKite (MPCK); this is a large aerostat $\left(250 \mathrm{~m}^{3}\right.$ of helium) that can carry a scientific payload of up to $60 \mathrm{~kg}$ [3, 62].

In this thesis an in-situ experiment is described, that is designed to simultaneously measure all aspects relevant to turbulent collision-coalescence of cloud droplets: droplet motions, droplet sizes, and properties of the turbulent carrier flow. The experiment is located in the German Alps, on top of the environmental research station Schneefernerhaus. Due to the local topography the wind tends to come from either east or west. Furthermore, at the experiment's altitude of $2650 \mathrm{~m}$, clouds naturally occur. This makes the measurement site suitable for the application of techniques that are commonly used in wind tunnel experiments. Droplet motions are measured using a particle tracking setup that is housed in a rigid, weather-proof box. The difficulty with field experiments like the one presented here, is that neither the droplet sizes nor the turbulent flow can be controlled. A technique is developed to measure the size of individual droplets based on their intensity as observed by the particle tracking experiment. The turbulent statistics are measured using a sonic anemometer that is installed close to the particle tracking setup. The experiment is finally used to measure droplet rms accelerations and the radial distribution function, conditioned on the Stokes number.

This thesis is organised as follows: first, an overview of the relevant theory (chapter 2) and of the experimental setup (chapter 3 are given. Then, in chapters 46 , the particle tracking experiment, the droplet sizing method, and the turbulence statistics are discussed. Finally, in chapter 7, a case study is presented, in which the rms accelerations and radial distribution functions on three different days are measured.

It must be noted the experiment that is presented here has the ability to compensate for the mean wind, but this ability is not exploited in this work. For more details, the reader is referred to appendix $D$. 


\section{Chapter 2}

\section{Theory}

Part of this chapter has been adapted from the author's previous work [6].

\subsection{Turbulence}

This section is based on Nieuwstadt [47] and Pope [48].

All incompressible Newtonian flows, including turbulence, are described by the Navier-Stokes equations:

$$
\frac{\partial \boldsymbol{U}}{\partial t}+\underbrace{(\boldsymbol{U} \cdot \nabla) \boldsymbol{U}}_{\mathrm{II}}=-\frac{1}{\rho} \nabla p+\underbrace{v \nabla^{2} \boldsymbol{U}}_{\mathrm{IV}},
$$

with $U$ the velocity at point $x$ in space, $p$ the pressure, and $\rho$ and $v$ the density and the kinematic viscosity of the fluid. Incompressibility is expressed as $\nabla \cdot U=0$.

Whether or not a flow becomes turbulent depends on the Reynolds number Re. This is a dimensionless number that is the ratio between the inertial forces and the viscous forces, and can be estimated from the corresponding terms in the Navier-Stokes equations, terms II and IV in equation (2.1). The Reynolds number is usually expressed as

$$
\operatorname{Re}=\frac{\mathcal{U} \mathcal{L}}{v},
$$


Table 2.1: The Kolmogorov scales for three typical dissipation rates found in cloud turbulence [65]. These values were computed using a kinematic viscosity of $1.83 \times 10^{-5} \mathrm{~m}^{2} \mathrm{~s}^{-1}$; this value is appropriate for the Zugspitze, where, due to the altitude, the air pressure and density are only $75 \%$ of those at sea level.

\begin{tabular}{cccc}
\hline$\epsilon\left[\mathrm{m}^{2} \mathrm{~s}^{-3}\right]$ & $\eta[\mathrm{mm}]$ & $\tau_{\eta}[\mathrm{ms}]$ & $u_{\eta}\left[\mathrm{cm} \mathrm{s}^{-1}\right]$ \\
\hline 0.001 & 1.57 & 135 & 1.2 \\
0.01 & 0.88 & 43 & 2.1 \\
0.1 & 0.49 & 14 & 3.7 \\
\hline
\end{tabular}

with $\mathcal{U}$ a typical velocity and $\mathcal{L}$ a typical length. Low Reynolds-number flows $(\operatorname{Re}<1)$ are dominated by viscosity and are laminar, while high Reynoldsnumber flows $(\operatorname{Re} \gg 1)$ exhibit strong non-linearity and are chaotic.

Turbulence is often described as a chaotic flow that is composed of eddies (vortices) of many different sizes, all of which interact with each other. Energy is put in at the largest scales, in the so-called energy-containing range. It then cascades to the smaller scales, which it can do as long as the inertial forces dominate the viscous forces. This defines the inertial subrange. As energy cascades to the smaller scales, the viscous forces get ever more important. At the smallest scale, the dissipative scale, the viscous forces finally dominate and energy is dissipated. This energy cascade was first described by Richardson [50].

At the large scale, energy is put into the cascade by interaction of the flow with its domain boundaries or by some volume stirring mechanism. At this scale the flow geometry has direct effect on the large scale motions of the flow. As energy cascades down to smaller scales, this information is lost. As a result a mathematical description of the smallest scales can only depend on the kinematic viscosity $v$ (unit: $\mathrm{m}^{2} \mathrm{~s}^{-1}$ ) and the rate at which energy flows through the cascade and finally dissipates, the dissipation rate $\epsilon$ (unit: $\mathrm{m}^{2} \mathrm{~s}^{-3}$ ). This is Kolmogorov's first similarity hypothesis. With only $v$ and $\epsilon$ three quantities can be formed that have units of length, time, and velocity:

$$
\begin{aligned}
\eta & =\left(v^{3} / \epsilon\right)^{1 / 4} \\
\tau_{\eta} & =(v / \epsilon)^{1 / 2} \\
u_{\eta} & =(v \epsilon)^{1 / 4}
\end{aligned}
$$

These are the Kolmogorov microscales [33] and they define the smallest scales present in turbulence. The Reynolds number at the Kolmogorov scale is 
$u_{\eta} \eta / v=1$, which shows that the eddies at that scale dissipate quickly due to viscous effects. Typical values for the Kolmogorov scales in cloud turbulence can be found in table 2.1

Scales within the inertial subrange are small enough that they are not affected by the boundaries of the flow, but are still too large to be affected by viscosity. As a result, the mathematical description of these scales can only depend on the dissipation rate $\epsilon$ and the size of the eddies of interest. This is Kolmogorov's second similarity hypothesis.

\subsubsection{Reynolds equations}

Turbulence is described by the Navier-Stokes equations 2.1 , but very little can be proved directly from these equations, not even the boundedness of its solution [44]. Turbulence is a stochastic phenomenon that must be described by statistical quantities. It is not possible to derive effective equations for these statistical quantities starting from the Navier-Stokes equations; this circumstance signifies the turbulence problem.

The most basic statistical quantity is the mean velocity field $\bar{u}=\langle\boldsymbol{U}\rangle$, which is governed by the Reynolds equations. These are obtained by averaging the Navier-Stokes equations. They are

$$
\frac{\partial \bar{u}_{i}}{\partial t}+(\bar{u} \cdot \nabla) \bar{u}_{i}=-\frac{1}{\rho} \frac{\partial}{\partial x_{i}} \bar{p}+v \nabla^{2} \bar{u}_{i}-\underbrace{\frac{\partial}{\partial x_{j}}\left\langle u_{i} u_{j}\right\rangle}_{\mathrm{V}},
$$

with $u_{i}=U_{i}-\bar{u}_{i}$ the fluctuating part of the velocity, $\bar{p}$ the mean pressure, and subscripts $i$ and $j$ indexing components of a vector. The Reynolds equations are similar to the Navier-Stokes equations (2.1), except for the Reynolds stress term (term V). This term introduces extra unknowns and for this reason the Reynolds equations cannot be solved directly. This is a manifestation of the turbulence problem.

The turbulent kinetic energy $k$ is defined as

$$
k \equiv \frac{1}{2}\langle u \cdot u\rangle .
$$

Pope [48, \$5.3] gives an evolution equation for $k$, from which the definition for the dissipation rate $\epsilon$ can be obtained:

$$
\epsilon \equiv v\left\langle\frac{\partial u_{i}}{\partial x_{j}} \frac{\partial u_{i}}{\partial x_{j}}\right\rangle=15 v\left\langle\left(\frac{\partial u_{1}}{\partial x_{1}}\right)^{2}\right\rangle
$$


where the latter equality holds for isotropic turbulence.

The rms velocity $u_{i}^{\prime}$ is the root-mean-square of the velocity fluctuations $u_{i}$. In isotropic turbulence $u_{1}^{\prime}=u_{2}^{\prime}=u_{3}^{\prime}=u^{\prime}$, and the turbulent kinetic energy is $k=\frac{1}{2}\left(u_{1}^{\prime 2}+u_{2}^{\prime 2}+u_{3}^{\prime 2}\right)=\frac{3}{2} u^{\prime 2}$.

\subsubsection{Structure functions}

Another statistical quantity is the structure function. Explicit expressions for the structure functions can be found based on theoretical considerations. They can easily be measured in experiments and are often used to verify that a given flow is in fact turbulent. Furthermore they can be used to estimate the dissipation rate.

The second-order structure functions are defined as:

$$
D_{i j}(r, t) \equiv\left\langle\left[u_{i}(x+r, t)-u_{i}(x, t)\right]\left[u_{j}(x+r, t)-u_{j}(x, t)\right]\right\rangle .
$$

In isotropic turbulence the structure functions $D_{i j}$ can be written in terms of the longitudinal structure function $D_{L L}$ and the transverse structure function $D_{N N}$ :

$$
D_{i j}(r, t)=D_{N N}(r, t) \delta_{i j}+\left[D_{L L}(r, t)-D_{N N}(r, t)\right] \frac{r_{i} r_{j}}{r} .
$$

According to Kolmogorov's second similarity hypothesis, for separations $r$ in the inertial subrange, the form of the structure function can only be a function of $\epsilon$ and $r$. The only dimensionally correct combination that can be formed is

$$
D_{L L}(r, t)=C_{2}(\epsilon r)^{2 / 3},
$$

with $C_{2}=2.12$ an empirically determined, universal constant. For the transverse structure function, it can be shown that in the inertial subrange

$$
D_{N N}(r, t)=\frac{4}{3} D_{L L}(r, t) .
$$

An example of second-order structure functions measured in wind tunnel turbulence is shown in figure 2.1. These structure functions are compensated, i.e. scaled by $(\epsilon r)^{2 / 3}$, so that they show a plateau in the inertial subrange. The second-order structure functions can be used to determine the dissipation rate of a turbulent flow. This is done by first scaling the structure functions with $r^{2 / 3}$, so that the inertial-subrange plateau appears. The dissipation rate is then determined from the height of the plateau. This method is used in chapter 6 


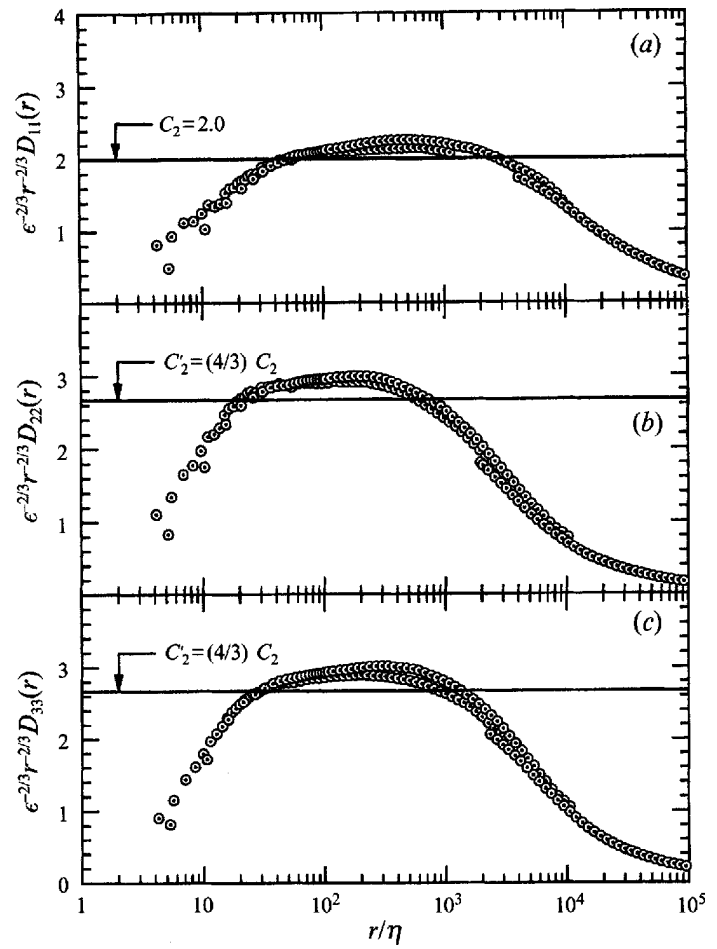

Figure 2.1: Second-order structure functions from wind tunnel experiments. The horizontal axis is scaled by the Kolmogorov length scale. These structure functions are compensated, i.e. the vertical axis is scaled by the inertial range energy $(\epsilon r)^{2 / 3}$. The plateaus correspond to the inertial range. (Source: Saddoughi and Veeravalli 54 . fig. 18].) 


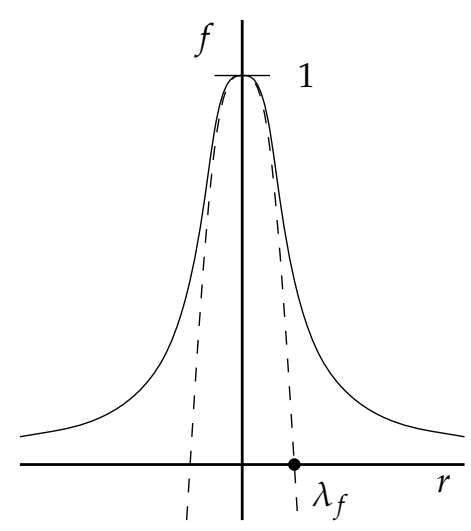

Figure 2.2: A sketch of the longitudinal velocity autocorrelation function showing the definition of the Taylor mircroscale $\lambda_{f}$. (Source: Pope [48. figure 6.7].)

\subsubsection{Two-point velocity autocorrelation}

A third statistical quantity is the velocity autocorrelation function. The velocity spectra (see next section) and a number of length and time scales are computed from this quantity. The two-point velocity autocorrelation functions are generally defined as:

$$
R_{i j}(r, t) \equiv\left\langle u_{i}(x+r, t) u_{i}(x, t)\right\rangle .
$$

In isotropic turbulence this function can again be written in terms of a longitudinal $(f)$ and transverse $(g)$ autocorrelation function:

$$
R_{i j}(r, t)=u^{\prime 2}\left(g(r, t) \delta_{i j}+[f(r, t)-g(r, t)] \frac{r_{i} r_{j}}{r}\right),
$$

A typical longitudinal autocorrelation function is sketched in figure 2.2. From the autocorrelation functions two length scales can be computed. The first is the integral length scale $L$. It is computed as the integral of the autocorrelation function:

$$
L_{11}=\int_{0}^{\infty} f(r, t) \mathrm{d} r .
$$

This is the longitudinal integral length scale; its transverse counterparts $L_{22}$ and $L_{33}$ are computed from $g$, and in isotropic turbulence they are equal to 
half of $L_{11}$. The integral length scale is the characteristic length scale of the large eddies.

The second length scale that can be computed is the Taylor length scale $\lambda$. It is computed from the zero-crossing of the parabola that osculates the autocorrelation's peak. This is shown in figure 2.2. Two Taylor length scales can be computed: $\lambda_{f}$ based on $f$ and $\lambda_{g}$ based on $g$. The Taylor length scale is intermediate in size between $\eta$ and L. It does not have a clear physical interpretation, but is very well-defined and is often used, in particular in the Taylor Reynolds number,

$$
\operatorname{Re}_{\lambda} \equiv \frac{u^{\prime} \lambda_{g}}{v}
$$

which is traditionally used to characterise grid turbulence. The Taylor scale is related to the dissipation rate, $\lambda_{g}^{2}=15 v u^{\prime 2} / \epsilon$, so that the Taylor Reynolds number can be written as

$$
\operatorname{Re}_{\lambda}=\sqrt{\frac{15 u^{\prime 4}}{\epsilon v}} .
$$

The velocity autocorrelation functions are related to the second-order structure functions as follows:

$$
D_{i i}(r, t)=2\left[R_{i i}(0, t)-R_{i i}(r, t)\right]
$$

\subsubsection{Velocity spectra}

The three-dimensional energy spectrum $E(\kappa)$ measures the kinetic energy at a wavenumber $\kappa$. In experiments it is difficult to measure, because it requires knowledge of the entire velocity field. The one-dimensional spectra $E_{i i}\left(\kappa_{1}\right)$ are much more accessible; they are defined as

$$
E_{i i}\left(\kappa_{1}\right) \equiv \frac{1}{\pi} \int_{-\infty}^{\infty} R_{i i}\left(\boldsymbol{e}_{1} r_{1}\right) e^{-i \kappa_{1} r_{1}} \mathrm{~d} r_{1}
$$

In the inertial subrange, $E_{i i}$ can only be a function of $\epsilon$ and $\kappa_{1}$. The only dimensionally correct combination of these is

$$
E_{11}=C_{1} \epsilon^{2 / 3} \kappa_{1}^{-5 / 3},
$$

with $C_{1} \approx 0.5$ an experimentally determined constant. This is the famous Kolmogorov $-\frac{5}{3}$ spectrum. 


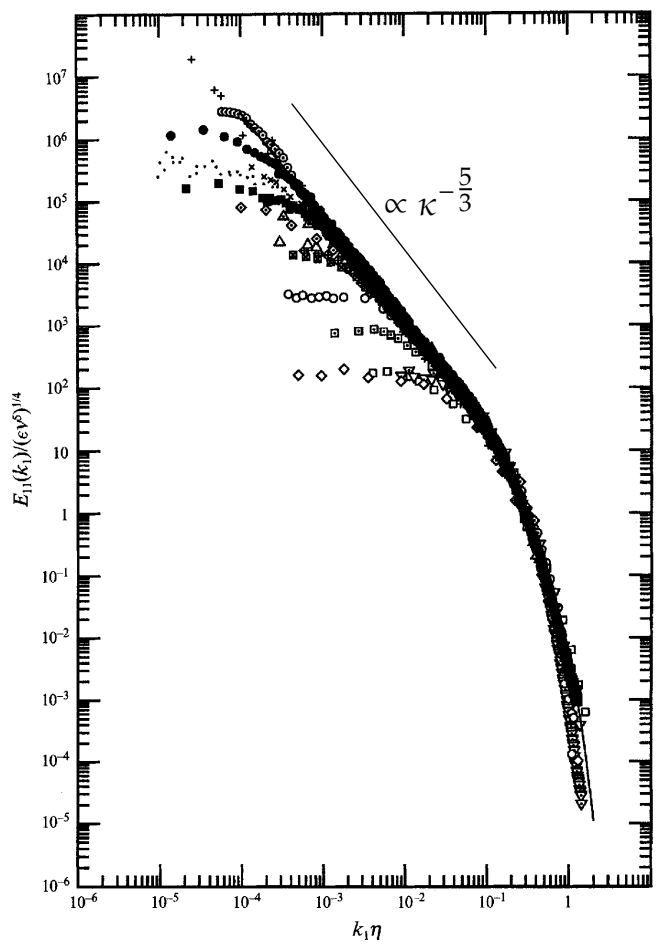

Figure 2.3: 1-D energy spectra from several experiments. The axes are scaled by the Kolmogorov length scale $\eta$ and the dissipative scale energy $\left(\epsilon v^{5}\right)^{1 / 4}$. The inertial subrange, in which the Kolmogorov $-\frac{5}{3}$ law holds, can readily be identified. (Source: Saddoughi and Veeravalli [54. figure 9].) 
A number of 1-D longitudinal $(i=j=1)$ energy spectra are shown in figure 2.3 The horizontal axis is scaled by the Kolmogorov length scale $\eta$; the vertical axis is scaled by the dissipative scale energy $\left(\epsilon v^{5}\right)^{1 / 4}$. The spectra clearly show the inertial subrange, in which the Kolmogorov $-\frac{5}{3}$ law holds. Furthermore the dissipative scale, in which the energy decreases much faster than $\kappa^{-5 / 3}$, can be seen for $\kappa_{1} \eta \gtrsim 0.1$. Due to the scaling the inertial subranges collapse, as do the dissipative ranges; this shows the universality of the Kolmogorov scales and the Kolmorogorov $-\frac{5}{3}$ spectrum. Finally the energy containing range can also be seen; it is where each curve levels off at the left hand side of the graph.

\subsection{Droplet kinematics}

For particle-laden flows the Navier-Stokes equations must be augmented by an equation of motion for the discrete particles. Furthermore terms must be added that account for the interplay between the fluid velocity field and the particle motion. For very dilute suspensions of point-like particles, the effect of particles on the fluid may be omitted (called one-way coupling), whereas for dense suspensions or large particles, the motion of the particle fraction reacts back on the fluid, (called two-way coupling). Even higher number densities may introduce particle-particle interactions, a regime known as four-way coupling. In clouds the number density is low, and one-way coupling can be assumed.

Maxey and Riley [41] derive the equations of motion for a small rigid sphere in a flow:

$$
\begin{aligned}
m_{p} \frac{\mathrm{d} v_{i}}{\mathrm{~d} t}= & \left(m_{p}-m_{f}\right) g_{i}^{(\mathrm{I})}+\left.m_{f} \frac{\mathrm{D} u_{i}}{\mathrm{D} t}\right|_{y(t)} ^{(\mathrm{II})} \\
& -\frac{1}{2} m_{f} \frac{\mathrm{d}}{\mathrm{d} t}\left\{v_{i}(t)-u_{i}[\boldsymbol{y}(t), t]-\left.\frac{1}{10} a^{2} \nabla^{2} u_{i}\right|_{y(t)}\right\}^{(\mathrm{III})} \\
& -6 \pi a \mu\left\{v_{i}(t)-u_{i}[y(t), t]^{(\mathrm{IV})}-\left.\frac{1}{6} a^{2} \nabla^{2} u_{i}\right|_{y(t)} ^{(\mathrm{V})}\right\} \\
& -6 \pi a^{2} \mu \int_{0}^{t} \mathrm{~d} \tau\left(\frac{\mathrm{d} / \mathrm{d} \tau\left\{v_{i}(\tau)-u_{i}[\boldsymbol{y}(\tau), \tau]-\left.\frac{1}{6} a^{2} \nabla^{2} u_{i}\right|_{y(\tau)}\right\}}{[\pi v(t-\tau)]^{1 / 2}}\right)^{(\mathrm{VI})}
\end{aligned}
$$

Here $m_{p}$ is the particle mass, $m_{f}$ is the displaced fluid mass, $a$ is the particle radius, $y$ is the particle position, $v$ is the particle velocity, $u$ is the undisturbed 
flow velocity, $\mu$ is the fluid's dynamic viscosity, and $g_{i}$ is the gravitational acceleration. The numbered terms are:

I buoyancy,

II local fluid acceleration,

III added mass term,

IV Stokes drag,

$\mathrm{V}$ the Faxén correction term, and

VI the Basset history term.

Several of these terms can be neglected. Since this study concerns droplets in air the particle density $\rho_{p}$ is much larger than the fluid density $\rho_{f}$, and hence $m_{p} \gg m_{f}$. Consequently terms II and III can be neglected.

The Faxén correction term (term V) accounts for forcing due to velocity gradients along the surface of the spehere. When the sphere is much smaller than typical scales in the flow, the Kolmogorov length scale $\eta$ in this case, this term can be neglected. In this study typical particle diameters are $40 \mu \mathrm{m}$ and $\eta=700 \mu \mathrm{m}$, so this term is neglected.

The Basset history term (term VI) accounts for the changing boundary layer around the sphere in an unsteady flow. Such boundary layers need time to develop, hence this term is called the history term. It has an $a^{2}$ prefactor, which is very small given typical particle diameters in this study and therefore this term can also be neglected. Equation 2.19) now reduces to:

$$
m_{p} \frac{\mathrm{d} v_{i}}{\mathrm{~d} t}=\left(m_{p}-m_{f}\right) g_{i}-6 \pi a \mu\left\{v_{i}(t)-u_{i}[\boldsymbol{y}(t), t]\right\}
$$

Taking into account that $m_{p} \gg m_{f}$, this can be rewritten as follows:

$$
\frac{\mathrm{d} v_{i}}{\mathrm{~d} t}=g_{i}-\frac{1}{\tau_{p}}\left\{v_{i}(t)-u_{i}[\boldsymbol{y}(t), t]\right\}, \quad \tau_{p}=\frac{m_{p}}{6 \pi a \mu},
$$

with $\tau_{p}$ the particle relaxation time. Whether or not the buoyancy term (term I) can be neglected, depends on the ratio of the settling velocity and turbulent fluctuations. The settling velocity $v_{T}$ can be found from the above equation by setting $\mathrm{d} v_{i} / \mathrm{d} t=0$ and $u_{i}=0$. This gives

$$
v_{T}=\tau_{p} g
$$

Typical cloud droplets have a radius $a$ of approximately $20 \mu \mathrm{m}$. For these droplets $\tau_{p}=5 \mathrm{~ms}$, so the settling velocity is at most $5 \mathrm{~cm} \mathrm{~s}^{-1}$, which is less 
than $5 \%$ of the typical $\mathrm{rms}$ velocity $u^{\prime}$. Therefore the buoyancy term can also be neglected.

The particle mass can be expressed in terms of particle density $\rho_{p}$ and diameter $d_{p}$, and substituted in the equation for $\tau_{p}$ :

$$
\tau_{p}=\frac{\rho_{p} d_{p}^{2}}{18 \mu} .
$$

Since $\rho_{p}$ and $\mu$ are constant $\tau_{p}$ only depends on the particle diameter.

The dimensionless number $\tau_{p} / \tau_{\eta}$ is called the Stokes number (St). It determines how fast a particle responds to changes in the surrounding flow. A low Stokes number, St $\ll 1$, means the particle responds immediately, and hence follows the flow exactly. Such particles are also called tracer particles or simply tracers. Particles with Stokes number $\sim 1$ or larger lag behind the flow field.

\subsection{Cloud droplet growth}

This section is based on review articles by Shaw [60], Devenish et al. [14] and Grabowski and Wang [24], and on the books by Wallace and Hobbs [67] and Pruppacher and Klett [49].

The formation and growth of cloud droplets is divided into three phases: activation, condensational growth, and collision-coalescence. The first phase, activation, starts when moisture in a supersaturated patch of air condenses onto solid, sub-micron aerosol particles (cloud condensation nuclei, or CCN), creating miniscule droplets. The growth rate of these droplets is a balancing act between the air's supersaturation, the concentration of dissolved materials, and the droplets' current size. Larger droplets have lower concentrations of salts, which causes growth to slow down or even stall. On the other hand, larger droplets have lower surface curvature, so their growth is less hindered by surface tension. Once droplets reach a certain critical size, the latter effect becomes dominant, and further growth becomes a runaway process. At this point the $\mathrm{CCN}$ is said to be activated.

As long as the air is supersaturated, humidity condenses onto the droplets and keeps adding to their volume. The rate at which this happens is inversely proportional to the drop's size, i.e. $a^{-1}$, and hence is less effective for larger droplets. For droplets with radii larger than $15 \mu \mathrm{m}$, condensational growth is deemed ineffective [14]. 
Finally, once the droplets get large enough to be sufficiently affected by their inertia and/or by gravity, they further grow through collisioncoalescence with other droplets.

The combined effect of these three phases is described by the evolution equation for the droplet size distribution. Let $f(a)$ be the number density of droplets with radii between $a$ and $a+\mathrm{d} a$, then

$$
\begin{aligned}
\frac{\partial f(a)}{\partial t}= & J(a)-\frac{\partial[\dot{a} f(a)]}{\partial a} \\
& -\int_{0}^{\infty} K_{12}\left(a, a^{\prime}\right) f(a) f\left(a^{\prime}\right) \mathrm{d} a^{\prime} \\
& +\frac{1}{2} \int_{0}^{a} \frac{a^{2}}{a^{\prime \prime 2}} K_{12}\left(a^{\prime \prime}, a^{\prime}\right) f\left(a^{\prime \prime}\right) f\left(a^{\prime}\right) \mathrm{d} a^{\prime} .
\end{aligned}
$$

The first term on the right side is a source term that models activation. The second term corresponds to condensational growth, with $\dot{a}$ the growth rate. The third and fourth term represent collision-coalescence. The third term models the decrease in number density of drops of radius $a$ due to collisioncoalescence with drops of radius $a^{\prime}$, while the fourth term models the increase in number density as a result of collisions between smaller droplets of radii $a^{\prime}$ and $a^{\prime \prime}=\left(a^{3}-a^{\prime 3}\right)^{1 / 3}$. The factor $1 / 2$ accounts for the fact that the integral counts collisions twice: once between drops with radii $a^{\prime}$ and $a^{\prime \prime}$, and once more with those radii swapped. $K_{12}$ is the collection kernel; it describes how many collision-coalescences per unit volume occur between droplets of radii $a_{1}$ and $a_{2}$.

For two droplets to collide and coalesce, three things need to happen: (1) they need to undergo a geometric collision, (2) the hydrodynamic interactions must allow the droplets to truly collide, and (3) the surface properties must allow coalescence to occur. The exact meaning of each of these is best described by considering the case of two droplets, with radii $a_{1}>a_{2}$, settling in quiescent air, as is done below. It must be noted, though, that these concepts also apply to turbulent collision-coalescence.

Settling droplets are said to undergo a geometric collision if their horizontal distance is less than $R=a_{1}+a_{2}$. The corresponding geometric collision crosssection is $\pi R^{2}$. This is shown in the left of figure 2.4. The proportion of droplet pairs that undergo geometric collisions is given by the collision kernel $\Gamma_{12}$, so that

$$
\left\langle\mathcal{N}_{12}\right\rangle=\left\langle n_{1}\right\rangle\left\langle n_{2}\right\rangle \Gamma_{12}
$$



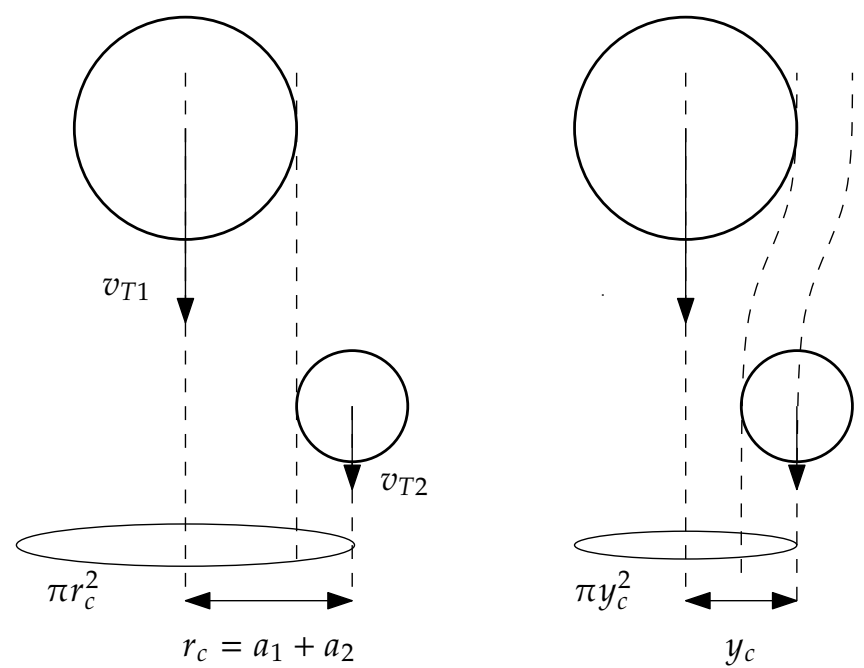

Figure 2.4: Two droplets with radii $a_{1}>a_{2}$ settling in quiescent air. In both figures the droplets will undergo a grazing collision. Left: without hydrodynamical interactions; the droplets are said to undergo a geometrical collision. The geometrical collision cross-section is $\pi R^{2}$, with $R=a_{1}+a_{2}$. Right: with hydrodynamic interactions. The larger droplet will push the smaller one aside, and as a result the effective collision cross-section $\pi y_{c}^{2}$ is smaller. Figure inspired by Pruppacher and Klett [49. figure 14.1]. 
with $\left\langle\mathcal{N}_{12}\right\rangle$ the average collision rate per unit volume, and $\left\langle n_{1}\right\rangle$ and $\left\langle n_{2}\right\rangle$ the average number densities of droplets with radii $a_{1}$ and $a_{2}$.

Each droplet produces a disturbance flow, which will generally repel other droplets coming near it; this is called hydrodynamic interaction (HI). As a result of the hydrodynamic interaction, two droplets that undergo a geometric collision, might not in fact truly collide. For them to truly collide, the horizontal distance between them needs to be at most $y_{c}$, with $y_{c}<R$. The effective collision radius $\pi y_{c}^{2}$ is therefore smaller than the geometric collision radius $\pi R^{2}$. The ratio of these is the collision efficiency $E_{12}=y_{c}^{2} / R^{2}$. The effect of hydrodynamic interaction on two settling droplets is sketched in the right of figure 2.4

Finally, if the droplets collide, a thin layer of air might get trapped between the two surfaces, preventing coalescence. This process is characterised by a coalescence efficiency $E_{12}^{\prime}$, which is better than 0.9 for cloud droplets 67 , fig. 6.22]. It is difficult to experimentally distuingish between the collision efficiency and the coalescence efficiency. Therefore, and because $E_{12}^{\prime} \sim 1$ for cloud droplets, the coalescence efficiency will not be considered any further in this work.

Given the collision kernel $\Gamma_{12}$ and the collision efficiency $E_{12}$, the collection kernel $K_{12}$ can be computed,

$$
K_{12}=\Gamma_{12} E_{12},
$$

so if accurate expressions for $\Gamma_{12}$ and $E_{12}$ were available, equation (2.24) could be used to predict the cloud droplet growth rate and the onset of precipitation. The aim of this thesis is provide experimental data on the collision kernel $\Gamma_{12}$.

\subsubsection{The collision kernel}

The collision kernel for droplets settling in a quiescent flow, is easy to determine. Consider a single droplet of radius $a_{1}$ that is settling in a collection of drops with radius $a_{2}<a_{1}$ and average number density $\left\langle n_{2}\right\rangle$. In this paragraph "droplet" refers to a droplet with radius $a_{1}$, while "drop" refers a droplet with radius $a_{2}$. Their respective settling velocities are $v_{T 1}$ and $v_{T 2}$. In a short period of time $\mathrm{d} t$, the droplet will collide with all drops present in the volume that is swept by the droplet in that time. The swept volume equals the collision cross-section $\pi R^{2}$ times the differential settling distance $\left|v_{T 1}-v_{T 2}\right| \mathrm{d} t$. This is the shaded volume in figure 2.5 The number of drops in that volume is thus $\left\langle n_{2}\right\rangle \pi R^{2}\left|v_{T 1}-v_{T 2}\right| \mathrm{d} t$. Up till now only a single droplet was considered. If there are many droplets with average number density $\left\langle n_{1}\right\rangle$, then the collision 


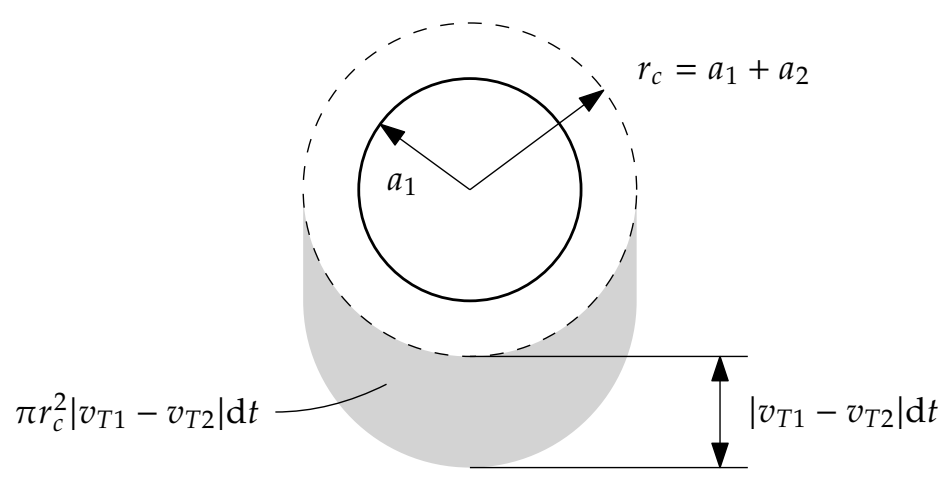

Figure 2.5: A droplet of radius $a_{1}$ settles in a collection of droplets of radius $a_{2}<a_{1}$. Their respective settling velocities are $v_{T 1}$ and $v_{T 2}$. The collision cross-section is $\pi R^{2}$, with $R=a_{1}+a_{2}$. All droplets of radius $a_{2}$ that are present in the shaded volume will collide with the droplet of radius $a_{1}$ within a short period of time $\mathrm{d} t$.

rate $\mathcal{N}_{12}$ per unit volume is given by

$$
\mathcal{N}_{12}=\left\langle n_{1}\right\rangle\left\langle n_{2}\right\rangle \pi R^{2}\left|v_{T 1}-v_{T 2}\right|,
$$

so that the collision kernel is $\Gamma_{12}=\pi R^{2}\left|v_{T 1}-v_{T 2}\right|$.

The collision kernel for two populations of droplets in a turbulent flow can be found following a similar approach [68]. The resulting collision kernel is

$$
\Gamma_{12}=2 \pi R^{2}\left\langle\left|w_{r}(r=R)\right|\right\rangle g_{12}(r=R),
$$

with $w_{r}$ the radial relative velocity (RRV), which is a measure for the speed at which droplets approach each other, and $g_{12}$ the radial distribution function (RDF), which measures the clustering of droplets. The radial relative velocity $w_{r}$ of a pair of droplets is given by $\boldsymbol{w} \cdot \hat{\boldsymbol{r}}$, with $\boldsymbol{w}=\boldsymbol{v}_{2}-\boldsymbol{v}_{1}$ the relative velocity, and $\hat{r}$ the unit vector corresponding to the separation vector $r=x_{2}-x_{1}$.

The radial distribution function is defined as

$$
g_{12}(r) \equiv \frac{N_{12, r} / \Delta V_{r}}{N / V},
$$

with $N_{12, r}$ the number of pairs of droplets with radii $a_{1}$ and $a_{2}$ separated by a distance $r, V_{r}$ the volume of a spherical shell with radius $r, N$ the total number of pairs in the system, and $V$ the system's volume [57]. In other words, it is the ratio of the actual number of pairs of droplets separated by a distance $r$, to what it would have been in a uniform suspension. 
To evaluate the collision kernel (2.28), the RDF and the RRV must be evaluated at droplet-touching distance $r=R$. While this is possible in numerical simulations, in experiments it is difficult to resolve droplets at this distance. The usual approach is therefore to assume some functional form for $g_{12}$, that can be fitted to experimental data for $r \gg R$ and extrapolated to $r=R$ [24]. This is the approach that is followed in chapter 7 


\section{Chapter 3}

\section{Setup}

Part of this chapter will be submitted as part of an article to Review of Scientific Instruments.

An overview of the experiment is shown in figure 3.1 In this chapter each of the main features of the experiment are briefly discussed, starting with its location (section 3.1). Then the seesaw is instroduced: this is the rail system that allows compensating for the mean wind, called seesaw for its ability to tilt. The camera box forms the heart of the particle tracking setup; it is not discussed here, but in chapter 4 , which is devoted to the particle tracking setup and method. In section 3.3 the control and data acquisition are discussed. Finally, in section 3.4 some of the other instruments that are available to us are introduced.

\subsection{Location}

The experiment is situated on top of the environmental research station Schneefernerhaus, at a height of $2650 \mathrm{~m}$ in the German Alps. Fig. 3.2 shows the topography of its immediate surroundings, while the inset of Fig. 3.1 shows the location of the experiment on top of the Schneefernerhaus. The Schneefernerhaus is located on a southern slope immediately south-west of Mt. Zugspitze. It looks over a relatively flat area, that is bordered to the north, south, and west by a mountain ridge. Directly to the west of the Schneefernerhaus the mountain ridge has a low spot due to wind erosion, which is called the wind hole. To the east the landscape slopes down into a valley. The wind 


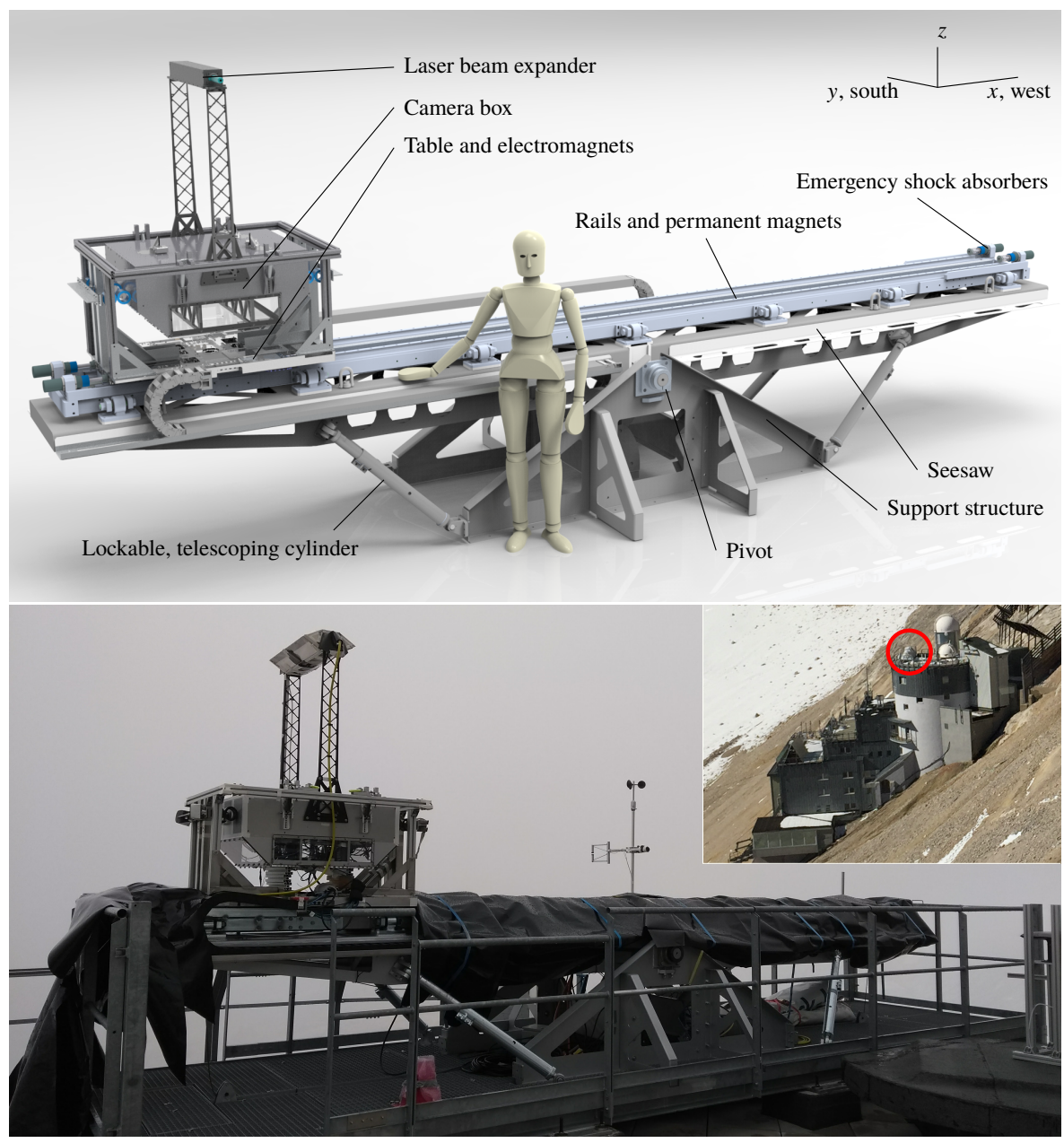

Figure 3.1: Top: A computer render of the experiment. The seesaw supports a pair of $6.5 \mathrm{~m}$ long rails, along which slides an aluminium table that supports the camera box. The seesaw, rails, and table together form a linear motor that can be used to make the LPT setup move with the mean wind. In case of failure of the motor or motor controller, shock absorbers at either end of the rails prevent damage to the apparatus. The seesaw can tilt by $\pm 15^{\circ}$, and can be locked in place using two pairs of stiff, lockable, telescoping cylinders. Bottom: A photo of the experiment. When this photo was taken, the seesaw was not in use. To protect it from unnecessary exposure to the elements, most of it was covered. Bottom inset: Location of the experiment on the Schneefernerhaus. This photo is taken from the East. 


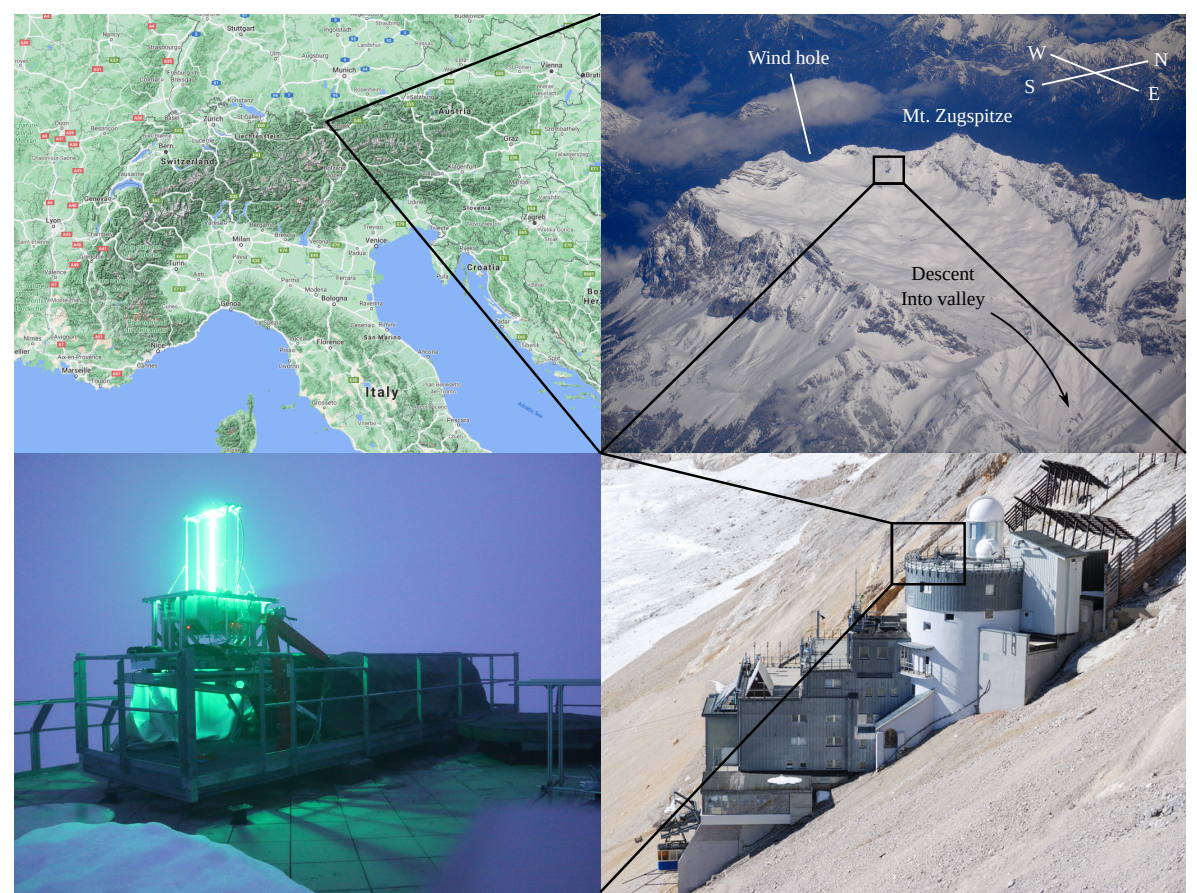

Figure 3.2: Topography near the Schneefernerhaus. The Schneefernerhaus is located in the German Alps, immediately south-west of Mt. Zugspitze, and looks over a large, flat area, that is bordered to the north, south, and west by a mountain ridge. Immediately to the west of the Schneefernerhaus the mountain ridge has a low spot, called the wind hole. To the east the landscape descends into a valley. Image credit: map: Google (2021, GeoBasis-DE/BKG (@2009), Inst. Geogr. Nacional; aerial: https://www.alpinforum. com/forum/viewtopic.php? $f=7 \& t=19048 \&$ start $=50$ 
hole and the valley determine the typical wind direction at the Schneefernerhaus: either easterly or westerly, but always blowing in between the wind hole and the valley.

Risius et al. [52] investigated suitability of this location for cloud turbulence research. Based on data of the German Weather Service (DWD) from 20002012 , they found the wind is indeed predominantly easterly or westerly. They furthermore found that clouds are likely to be present more than $25 \%$ of the time in summer (April-September), with a peak of $30 \%$ in July. Finally they measured turbulence statistics using five sonic anemometers. Typical dissipation rates obtained were $1 \mathrm{~cm}^{2} \mathrm{~s}^{-3}$ to $100 \mathrm{~cm}^{2} \mathrm{~s}^{-3}$, the mode of the Taylor Reynolds number was $\sim 3000$, and in terms of isotropy the flow was found to be similar to laboratory flows. Siebert et al. [61] did further measurements to characterise the cloud-turbulence interaction at the Schneefernerhaus. They again found that the small-scale turbulence is close to homogeneous and isotropic, and the cloud-turbulence interactions are comparable to those in free-atmospheric clouds under continental conditions. They concluded that, together with the results of Risius et al. [52], the Schneefernerhaus is a suitable location for Langrangian measurements of cloud droplets in turbulence.

\subsection{Seesaw and vibration damping}

The seesaw allows us to compensate for the mean wind. It supports the rails on which the table slides; see Fig. 3.1. It has been designed for velocities up to $7.5 \mathrm{~m} \mathrm{~s}^{-1}$ and accelerations up to $28 \mathrm{~m} \mathrm{~s}^{-2}$. It also withstands emergency stops, which occur for instance if the linear motor fails and the table runs into the endstops; in such events the deceleration can reach up to $230 \mathrm{~m} \mathrm{~s}^{-2}$.

A sliding table is mounted on a pair of rails on the seesaw. It is driven with a linear motor that is part of the Bosch-Rexroth IndraDyn L series, a series of industrial three-phase synchronous motors. The motor is controlled by a Bosch-Rexroth IndraDrive $C$ series controller, which is installed in a cabinet in the laboratory, two floors below the experiment. At its heart it has a set of cascaded PID controllers that control the motor position by setting the motor current, taking maximum velocity, acceleration, and jerk (third derivative of the position with respect to time) into account. The IndraDrive controller allows automated, real-time control, either by loading a motion control programme into it, or through a real-time bus such as CANopen.

Moving the LPT setup over rails causes vibrations that are detrimental to the particle tracking. To prevent these vibrations from reaching the camera box, the box is suspended from four extension springs Z-195I made by 


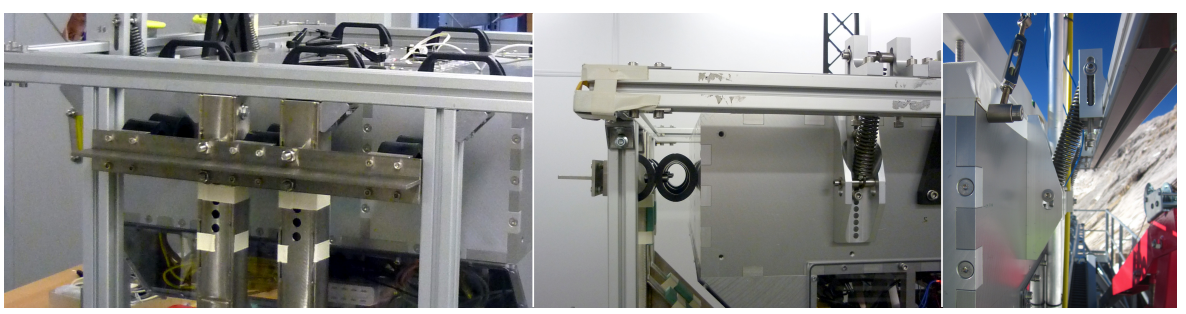

Figure 3.3: The camera box's suspension and buffers. Left: the buffers are adjusted, so when accelerating or decelerating, they push the box at the height of its centre of mass. Middle: overview of the springs and buffers; this viewing angle is comparable to the one in Fig. 3.1 Right: the camera box is suspended with extension springs. The springs are adjusted so that the camera box is level, and the spring attachment points are level with the box's centre of mass.

Gutekunst Federn with spring constant $6.07 \mathrm{~N} / \mathrm{mm}$ and unstressed length $149 \mathrm{~mm}$. The height of the spring attachments to the box can be adjusted to be at the height of the box centre of mass, which prevents the box from tilting during acceleration and deceleration. To limit the amplitude of the swing of the camera box during the acceleration and deceleration phase, the box is further constrained by six pairs of rubber buffers (see Fig. 3.3. The buffers' height can be adjusted so that they are level with the box's centre of mass.

The seesaw has not been used for the results presented in this thesis. A further discussion of the seesaw and some of the effort that went into it is therefore omitted here, but can be found in appendix $D$

\subsection{Control and data acquisition}

The experiment is controlled by a small computer cluster that is installed in a laboratory two floors below the apparatus. It consists of a main node, 6 compute nodes, and 2 storage nodes, each of which is connected to $10 \mathrm{Gbit}$ Ethernet switches, and contains $35 \mathrm{~TB}$ of storage. The cameras are also connected to the $10 \mathrm{Gbit}$ network, through which they are controlled and read out. The main node runs a Python code that controls and triggers the cameras, controls the box's window shutters, monitors the box's environmental parameters, and controls the laser. After an experiment the compute nodes download videos from the cameras to their internal hard drives. This takes approximately 40 seconds, which is the limiting factor for the experiment's 
repetition rate. The videos are then copied to both storage nodes. After a measurement campaign the disks from the second storage node are taken out and transported to Göttingen, where data analysis takes place.

\subsection{Other instruments}

- Trumpf Laser. This fibre-coupled laser is used to illuminate the measurement volume. It is controlled by a Python code running on the cluster's main node, using OPC-UA [20] over Ethernet.

- Thies Clima 3D sonic anemometer. This sonic anemometer provides high resolution wind velocity measurements. It is used in chapter 6 to determine the turbulence statistics.

- Lufft WS500-UMB compact weather station. This weather station measures wind speed and direction, temperature, and humidity, all of which is logged at a very low temporal resolution $(0.1 \mathrm{~Hz})$. Data from this weather station is used to asses the weather conditions. The Lufft weather station is interrogated by a Python code running on the cluster's main node, using a binary protocol over RS- 485 .

- Artium PDI-FPDR PDI probe. This PDI probe measures droplet size and a single velocity component. The probe has been used on all measurement campaigns. During the last measurement campaign it was noticed that its windows tend to get wet, and as a result the data quality is not reliably high. Furthermore the probe's software is painful to work with. Data from this probe was meant as a reference for the droplet sizing method that is developed in chapter 5, but for aforementioned reasons was not used. 


\section{Chapter 4}

\section{Particle tracking}

Part of this chapter will be submitted as part of an article to Review of Scientific Instruments.

\subsection{Setup}

\subsubsection{Camera Box}

The camera box is a custom-built, vibration-damped aluminium box housing the high-speed cameras used for the Lagrangian particle tracking (see Fig. 4.1. The box has been designed in such a way to minimize its total weight and cross-sectional area exposed to the wind, while at the same time being extremely rigid and able to fit three Vision Research v2511 cameras with their corresponding optics. Further streamlining of the box would have added a lot of complexity in terms of fabrication, usability, and serviceability, but if the current shape of the box proves to be problematic, lightweight streamlining elements, made out of e.g. styrofoam, can be added. The box's main components are 24 aluminium parts providing the rigid skeleton, 3 window sub-assemblies through which the cameras observe the measurement volume, and 6 transparent polycarbonate plates that provide visual and manual access to the box in case of malfunction. Its external dimensions are $930 \mathrm{~mm} \times 720 \mathrm{~mm} \times 360 \mathrm{~mm}$ and internally the box is subdivided into three upper sections containing one camera each, and three lower sections which contain the camera power supplies, Ethernet and trigger cables, cooling hoses, Arduino control unit, and temperature, humidity, and acceleration sensors. 


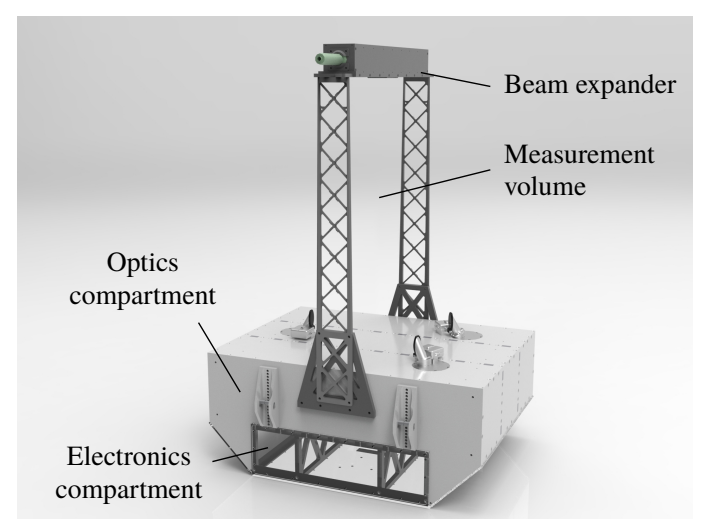

Figure 4.1: Rendering of the camera box and laser beam expander. The top compartment of the box houses the three cameras, optics, mirrors, and window sub-assemblies; it is completely made of aluminium to provide stiffness. The bottom compartment houses power supplies and control electronics; it has acrylic glass windows that provide visual access and can easily be removed for manual access.

The maximum weight of the camera box is in principle limited by the maximum payload that can be moved by the seesaw motor. However, more restrictive practical limits were imposed by the need to carry the box up to the roof manually through a narrow staircase which allowed only two people to carry the box at a time. For this reason, the weight of the box without cameras or beam expander (see below) was kept below $60 \mathrm{~kg}$. Even so, should the movable table crash into the emergency shock absorbers, very large forces would be generated both at the braces keeping the box above the table, and at the pillars fixing the seesaw to the roof. This lead to the current orientation of the box with its longest side parallel to the direction of motion. It was also considered to have the box stand upright, with its longest side vertical, however in such an orientation the torque applied on the table during a crash into the emergency shock absorbers would put a too large strain on the system.

The disadvantage of the current box orientation is that the optical access windows, which separate the inside and outside of the camera box, are more exposed to the effects of precipitation. A two-tier mechanism was designed to lower the risk of a water droplet residing in the optical path and thus deteriorating the quality of the images obtained. Each window is mounted at the lower end of a short tube (see Fig. 4.2), with the inner diameter of $24 \mathrm{~mm}$ just large enough to not interfere with any of the light rays between the camera 


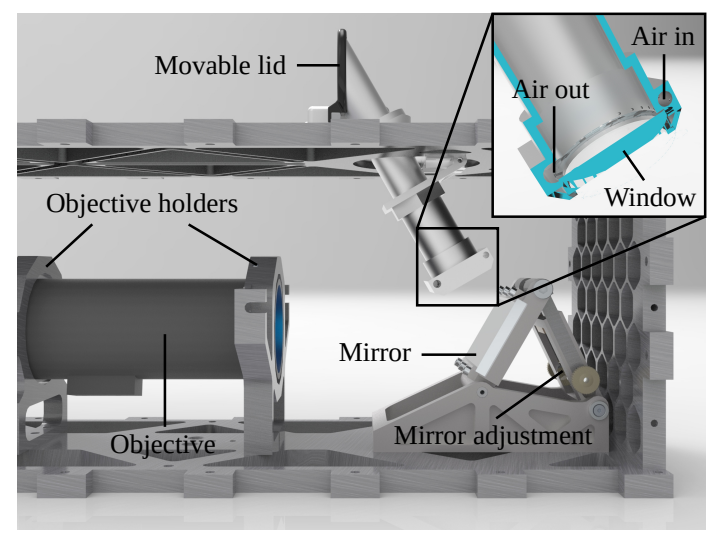

Figure 4.2: Side view of the camera objective held in place by the two holders, the adjustable mirror assembly and the optical access assembly. The top of the optical access assembly is closed off with a motorised movable lid. Inset: a section of the bottom of the optical access assembly. The window is located at the bottom of this assembly.

apertures and the measurement volume. The tubes are oriented along the line of sight of the cameras, that is at $30^{\circ}$ with respect to the vertical. Their upper ends are cut vertically to decrease the chance of a raindrop entering the tube, and are capped by movable lids. The lids open only for the duration of each video capture and close during the much longer duration of the data transfer. Moreover, a powerful stream of air is injected at the higher end of each window to remove any droplets that might still make their way through the tube. On the lower end of each window the air and any liquid collecting there is sucked out and removed from the camera box. To aid the water removal process, the windows are coated with a hydrophobic coating.

The box is supplied with a steady small stream of dry air to prevent water condensation on the underside of the windows. Currently the box is not temperature controlled. On a typical cloudy day when data can be taken, the outside temperature is between $0{ }^{\circ} \mathrm{C}$ to $5^{\circ} \mathrm{C}$ and the temperature within the box equilibrates to $30^{\circ} \mathrm{C}$ to $45^{\circ} \mathrm{C}$ after a few hours of operation, depending on the mean wind speed. These temperatures are within the operation range of the cameras, which is from $-10^{\circ} \mathrm{C}$ to $50^{\circ} \mathrm{C}$, as is the typical minimum inside temperature at dawn, so no cooling or heating is required for operation. It is possible that on a warm day with low wind speed the inside temperature could get too close to the upper operational limit, so there 

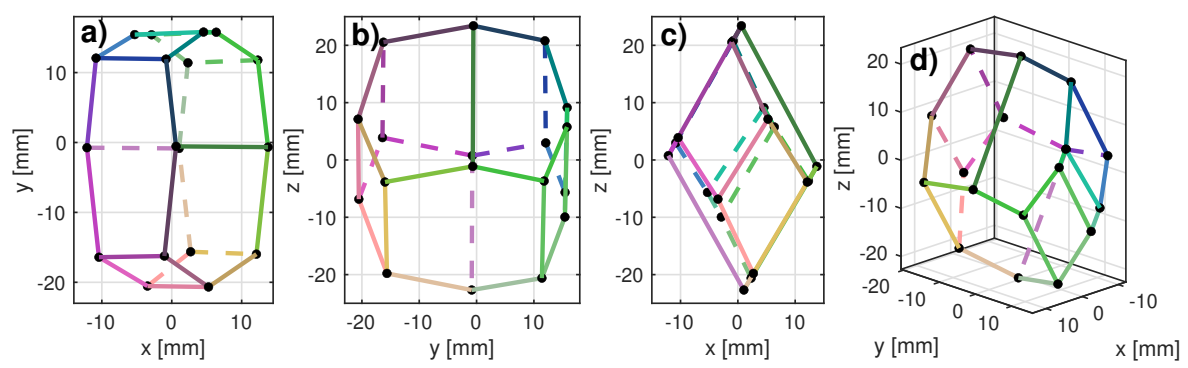

Figure 4.3: Measurement volume of the particle tracking setup, defined as the set of points visible by all three cameras, as seen from (a) the top, (b) the front (west), (c) the side (north) and (d) a general direction. The lines have the same colours between all figures; this might help in interpreting the various views.

are cooling channels running through the camera base plates which can help bring the inside temperature down; however the use of this cooling system has not been necessary so far. The temperature fluctuations do affect the camera calibration, but the changes are small enough to be removed by self-calibration without any adverse effects on the data quality.

\subsubsection{Optics}

The ideal particles for particle tracking are so small, that each of the multiple glare points that a single particle has, overlap on the camera sensors. The distance between the glare points is proportional to magnification $\times$ particle size, and since the size of the cloud droplets cannot be controlled, an optical system with a small magnification should be used. But to see droplet pairs at distances where they nearly touch each other, a large magnification is required. The present experiment has a magnification of $28 \mu \mathrm{m} \mathrm{px}^{-1}$, which means that if diffraction effects are ignored, a typical cloud droplet is of the order of $1 \mathrm{px}$ on the sensor. To provide the desired level of magnification, each camera is equipped with two $2 \times$ teleconverters and a Nikon $200 \mathrm{~mm}$ objective. To prevent sagging and any lateral motion of the objectives during box movement, each objective is supported on one end by a finely adjustable clamp ring and on the other end by three plastic adjustment screws. The cameras are also firmly bolted to the base plates underneath them.

To adjust the viewing angle of the cameras, each upper section also contains an $85 \mathrm{~mm} \times 60 \mathrm{~mm}$ mirror housed within a custom-built mirror holder (see Fig. 4.2. The holder can be rotated along a vertical axis and the mirror tilt can 
be finely adjusted using a fine-threaded adjustment screw. Once the desired rotation and tilt of the mirror is reached, this orientation can be kept in place by tightening a set of fixing bolts. The disadvantage of this holder design is that all adjustments need to be performed manually before the box is closed off.

The resulting measurement volume is diamond-shaped and measures about $16.6 \mathrm{~cm}^{3}$; see Fig. 4.3 . If one allows particles to be triangulated by less than 3 cameras, the usable volume is much larger, however in a large portion of it the particles are so much out of focus that they are hard to detect and their positions are obtained with large uncertainty.

\subsubsection{Illumination}

The requirement of a typical particle residence time on the order of a Kolmogorov time, with its most likely value of $30 \mathrm{~ms}$, and typical fluctuating velocity of roughly $2 \mathrm{~m} \mathrm{~s}^{-1}$, led to a choice of a rather large measurement volume with the largest horizontal diameter of around $35 \mathrm{~mm}$.

Achieving a depth of field comparable with the dimensions of the measurement volume, while simultaneously maintaining acceptable levels of signal to noise ratio even at the lower end of the droplet size range of interest across the measurement volume, requires a very powerful source of light. We use a Trumpf TruMicro 7240 laser with a wavelength of $515 \mathrm{~nm}$, a maximum pulse energy of $7.5 \mathrm{~mJ}$, and a pulse length of $300 \mathrm{~ns}$. Although at higher repetition rates the laser can achieve a light power output of $300 \mathrm{~W}$, at the lower repetition rates it is limited by the maximum pulse energy and thus for our purposes (i.e. sampling rate of $10 \mathrm{kHz}$ ) the light power output is effectively $75 \mathrm{~W}$.

The beam coming out of the laser head first passes through a diverging lens in order to reach the desired beam diameter within the small amount of space available in the beam expander. It then passes through a converging lens which makes it nearly parallel with diameter of approximately $39 \mathrm{~mm}$. The beam finally passes through an optical diffuser, the function of which is to smooth the dependence of the intensity of scattered light on the scattering angle, and thus simplify the process by which the droplet size is deduced. As the beam leaves the beam expander, it is clipped from both sides so it becomes narrower in the $x$-direction. By doing so it fits the shape of the measurement volume better; in particular the volume that is illuminated but not seen by all cameras, is reduced. The beam's profile at the measurement volume is shown in Fig. 4.4 There, the relative light intensity within the laser beam is plotted as a function of the position within its cross-section. The intensity data were 


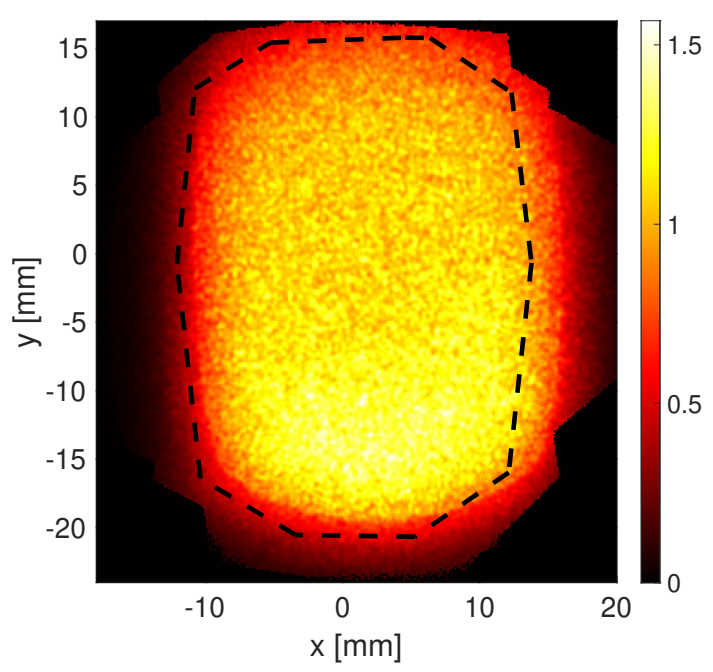

Figure 4.4: Dependence of the relative laser beam intensity on the location within its cross-section. The maximum extent of the measurement volume is indicated by the dashed line.

obtained from the tracking data by comparing instantaneous particle intensity with its mean value over its whole trajectory. Thus, data are available only for positions within view of at least two cameras, where triangulation is possible. The profile is nearly flat, with a slight asymmetry most likely due to a slight offset of the laser head from the optical axis of the other optics, and a smooth decrease of intensity at the edges due to the diffuser.

\subsubsection{Camera positions and viewing angles}

The amount of light is still a major limiting factor, so the geometry of the camera orientations with respect to the laser beam was adjusted to obtain the most favourable image quality whilst keeping the camera aperture diameter constant. The decisive parameter that sets the limits on maximum tractable seeding density and the uncertainty of the triangulated particles' three-dimensional position, is the mean variance of the positioning error of particle images on the sensor, $\sigma_{2 \mathrm{D}}^{2}$. For a given image, $\sigma_{2 \mathrm{D}}^{2} \sim \sigma_{N}^{2} / \mathcal{I}^{2}$, where $\sigma_{N}^{2}$ is the total variance of camera thermal and shot noise and $\mathcal{I}$ is the total intensity of the given particle image on the sensor. In this experiment, the dominant source of noise is usually the shot noise, so $\sigma_{N}^{2} \sim \mathcal{I}$. Thus, for fixed 
aperture size and camera orientation, $\sigma_{2 D}^{2} \sim 1 / \mathcal{I}$.

The image intensity is sensitively dependent on the scattering angle, that is the angle between the laser beam direction and the camera viewing axis. Although the dependence is non-monotonic for water droplets due to constructive and destructive interference between the refracted and reflected beams that reach the aperture, due to the use of a diffuser and a finite aperture size, these variations are mostly smoothed out. The dependence of the scattered light intensity on the scattering angle $\theta$ can then roughly be modelled as exponential: $\mathcal{I}(\theta) \sim \exp \left(-c_{\theta} \theta\right)$ with $c_{\theta} \approx 3.55 \mathrm{rad}^{-1}$ so that the intensity roughly halves with increase of $\theta$ by $11^{\circ}$. The rate constant $c_{\theta}$ was obtained from a fit to Lorentz-Mie scattering curves.

While making the scattering angle as small as possible will generally keep the particle image intensities large, it is not optimal for minimising the triangulation error due to the resulting geometry of the camera orientations: when all camera view axes are at a small angle with respect to the beam, they are necessarily also at a small angle to each other, and a small change in the particle image position on a given sensor can lead to a large shift in the component of the triangulated 3D-position along the laser beam direction. This geometric effect can be modelled by assuming the cameras are placed symmetrically with respect to the laser beam, at equal distances from the measurement volume, so that the angle between the view axes of any camera pair is the same, and that each camera view axis is at an angle of $\theta$ to the laser beam. It can be shown that the components of the three-dimensional particle position error variance are $\sigma_{x}^{2}=\sigma_{y}^{2} \sim \frac{2}{3} \sigma_{2 D}^{2} /\left(1+\cos ^{2} \theta\right)$ and $\sigma_{z}^{2} \sim \frac{1}{3} \sigma_{2 D}^{2} / \sin ^{2} \theta$. Here, the $z$-direction points along the laser beam. Thus, while small $\theta$ decrease the variance of the position error components perpendicular to the laser beam, in the direction along the beam small $\theta$ lead to a catastrophic amplification of the error.

Combining the effects of camera orientation geometry and the scattering light intensity yields

$$
\begin{gathered}
\sigma_{x}^{2}=\sigma_{y}^{2} \sim 2 \exp \left(c_{\theta} \theta\right) /\left(1+\cos ^{2} \theta\right) ; \\
\sigma_{z}^{2} \sim \exp \left(c_{\theta} \theta\right) / \sin ^{2} \theta .
\end{gathered}
$$

Optimising the total variance $\sigma_{x}^{2}+\sigma_{y}^{2}+\sigma_{z}^{2}$ leads to an optimal angle of $\theta=22.6^{\circ}$.

In order to accurately measure settling effects and the vertically-conditioned radial distribution function, a high accuracy in the vertical direction is needed. As the laser beam is pointing nearly vertically, $\sigma_{z}^{2}$ can be optimised, which leads to an optimal angle of $\theta=29.4^{\circ}$. The geometry of the camera box is designed for intended camera view axes at $30^{\circ}$ to the vertical. In reality, 
due to a slightly asymmetric placement of the measurement volume above the camera box, the actual view axes are at $(30.4 \pm 0.4)^{\circ}$ to the vertical.

It should be noted that the considerations of position error variance apply only to the particles with image intensity low enough to not saturate the sensor pixels. In our case, the smallest drop which can possibly lead to saturation (grayscale level of near 4096) when perfectly in focus has diameter of approximately $23 \mu \mathrm{m}$. This number was obtained using the droplet sizing method described in chapter 5 , combined with a model for a droplet's point spread function. For larger droplets, the optimum angle would be closer to the one obtained by geometric considerations only, which is $54.7^{\circ}$ (cameras view axes perpendicular to each other).

\subsection{Method}

During the winter the camera box and cameras are in Göttingen. For the measurement season, that typically runs from May till September, they are transported to the Schneefernerhaus. At the beginning of the season the camera box is assembled and put on the roof of the Schneefernerhaus. It is removed and partly disassembled at the end of the season. Calibration is done regularly, typically once every measurement campaign, so that if the optics lose alignment, whatever the reason may be, it is discovered early. Box assembly, calibration, and the procedure for a single experiment are described below.

\subsubsection{Start of season}

1. In the laboratory at Schneefernerhaus: put cameras, objectives, mirrors in the camera box and secure everything. Install the calibration rig. Focus the cameras, and align the mirrors. Once satisfactory alignment is reached, remove the cameras and objectives. This last step reduces the weight of the box, so it can be carried up the stairs to the roof.

2. Carry the box to the roof, and put it on the seesaw.

3. Put the cameras and objectives in the box and secure them. Connect the cameras to 10 Gbit Ethernet, BNC trigger cable, BNC frame sync cable, BNC time code cable, and power. The frame sync cable goes to the laboratory, so the cameras can be synchronised with the Trumpf laser.

4. Connect Arduino. The Arduino triggers the cameras. It is controlled via a USB-extender from the laboratory. 
5. Calibrate (see below).

6. Put the top plates and acryllic side panels on the camera box. Seal all seems with duct tape.

7. Start flow of clean, compressed air into the box. A small amount of airflow keeps the humidity in the box low. The box is not sealed well enough for pressure to build up.

\subsubsection{Calibration}

Calibration is typically a two-person job. One person is in the lab to control the cameras; the other is on the roof to move the calibration plate.

The calibration plate is a custom plate made of milky-white acrylic. One side of the plate is coated in a few layers of black spray paint, after which a hole pattern is drilled through the paint layer with a CNC milling machine. The plate is backlit with either an LED flashlight, sunlight, or the Trumpf laser. The milky-white acrylic causes the markers to be perfectly diffuse. The layers of black paint allow barely any light through. The resulting calibration images are patterns of very high contrast, white-on-black circular markers, with barely any structure in the markers.

The calibration proceeds as follows:

1. On the roof: install the calibration rig. Move the calibration plate to $500 \mathrm{~mm}$ above the camera box.

2. In the laboratory: connect the frame sync cable to a signal generator set to $100 \mathrm{~Hz}$. Configure the exposure time of each camera so that the resulting images have a high contrast.

3. Record calibration images in increments of $2 \mathrm{~mm}$. Images are taken at all positions at which all three central (fiducial) markers are visible on all cameras.

A Python script helps to take the calibration images and save them with the correct filenames.

\subsubsection{Single experiment}

1. In the lab: ensure the frame sync cable is connected to the Trumpf laser. 
2. Switch the laser on. Without this, the laser is not guaranteed to produce a frame sync signal, and the cameras will not be able to perform a black reference.

3. Black-reference the cameras. This "learns" each camera what the black level is. The sensors' dark currents can change as a result of changing camera box temperature. This step takes about 1 second.

4. Trigger the cameras, and wait for them to fill their buffers. This step takes between 2 and 4 seconds, depending on camera frame rate.

5. Switch the laser off, and instruct the cluster compute nodes to download the camera images. This step takes about 40 seconds.

6. If so desired, repeat from step 2.

Typically many experiments in a row are performed. To this end the above process is fully automated using a Python script.

\subsection{Data analysis}

A particle tracking code's aim is to find for each particle its position as a function of time. Most particle tracking codes approach this problem as follows:

1. Per frame, per camera: find 2-D positions of particle images. This is done by first finding the local intensity maxima, and then fitting two Gaussians to pinpoint the centre of the particle image with subpixel accuracy. One Gaussian is fitted to the central pixel and its two horizontal neighbours, and the other to the central pixel and its two vertical neighbours. This can be done analytically, i.e. no iteration is needed, and is therefore very fast. This typically yields an accuracy of 0.1 pixel.

2. Per frame: stereomatching, i.e. given the 2-D positions of all particle images on all cameras, find the particles' 3-D positions. To do so, for each particle image on the first camera a corresponding line of sight is cast. Then, on the second camera, all particle images that are close to this line are added to a list of candidates. For each candidate a 3$\mathrm{D}$ position can now be computed, and information from the third and fourth camera (if these are present) is used to see which of the candidates corresponds to a real particle. 
3. Per frame: tracking, i.e. given the 3-D positions of all particles, link each particle to the same particle in the previous frame or frames. Information from previous frames is used to predict where a particle is most likely to be at in the current frame. Sometimes two particles are found at the predicted position, in which case a tie-breaker method is needed.

(Source: [64, p. 789-792].)

While the above explanation is an oversimplification, it serves well to highlight two main issues. First, it is assumed in step 1 that particles appear on the camera sensors as compact, Gaussian spots. "Compact", here, means that they are at most a few pixels in diameter. In reality in-focus particles are imaged as Airy discs, the centres of which are well approximated as Gaussians. However, as soon as the particles get too far out of focus, they may appear as hollow rings, i.e. the intensity in the centre is lower than at the edges. The position of such particle images cannot be found by simple methods such as the one described in step 1 above.

The second issue is related to the seeding density. For low seeding densities the above algorithm works well. However, for increasing seeding densities, the particle images start to overlap. This has many implications: first of all, if two particle images overlap, only one will be found in step 1 . Secondly, step 2 must now be made to tolerate missing particle images. As a result the chance to find a particle where there isn't any increases, leading to ghost particles. Finally, step 3 must be modified to identify and discard these ghost particles.

An improvement is offered by the Shake-The-Box algorithm [58]. For particles that are known to the algorithm, the order of operations above is reversed: first, information from previous frames is used to predict particle positions. The predicted positions are then used as input to a "shaking" step: all predicted positions are used to create a simulated image for each camera. These are compared to what is recorded by the cameras, and the predicted positions are wiggled around ("shaken") to minimise the difference. After this is done for all particles, the simulated images are subtracted from what the cameras recorded; the result should contain only particle images of new particles, which are far fewer in number.

The advantage of using simulated images, is that overlapping particle images appear as brighter spots than non-overlapping particle images. The use of brightness information in the shaking step allows the Shake-The-Box algorithm to correctly deal with overlapping particle images. However, to create simulated images, the optical transfer function (OTF) or point-spread function (PSF) of the optical system must be known.

Due to large measurement volume used in this experiment, there can be up 
to $10^{4}$ tracked particles in the measurement volume at a single time. Although $10^{4}$ particles, which translates to 0.01 particles per pixel, a standard measure of tracking feasibility, is not very high by modern standards, the actual number of tracked images on each sensor is up to 2.5 times as high due to the shallow view angle with respect to the laser beam. Furthermore, due to the short depth of field compared to the size of the measurement volume, an inevitable consequence of working with the given amount of illumination, many of the images are badly out of focus. Thus the total area of all the tracked images may be several times higher than the total sensor area.

Due to the high seeding density and the badly focused particle images, a conventional particle tracking code is not suitable for analysing the particle videos from this experiment. Instead, a new code is used, called Low Light $L P T\left(\mathrm{~L}^{3} \mathrm{PT}\right)$, that is currently being developed by Dr. Moláček. The code is inspired by the Shake-The-Box algorithm. The largest similarity is in the use of a shaking step, but it differs in that much more computational cost is expended to increase both accuracy and yield. Specifically, particular care is taken to extract as many particle images as possible, even if they belong to particles outside the measurement volume, since this improves the accuracy of determining the location and intensity of overlapping particle images.

\subsection{Verification}

The particle tracking code is verified by analysing synthetic data. To this end, first a kinematic simulation is used to generate synthetic particle trajectories. These trajectories are then used to generate synthetic videos. Finally, the videos are processed with $\mathrm{L}^{3} \mathrm{PT}$, and the resulting trajectories are compared with the synthetic trajectories.

The author generated the synthetic trajectories; the verification itself was performed by Dr. Moláček and is not documented here.

\subsubsection{Kinematic simulation}

The synthetic trajectories are generated with a kinematic simulation (KS; see e.g. [22, 45, 46]). A kinematic simulation was chosen, because it has low computational cost, and yet reproduces some of the features of turbulent flows, such as particle clustering. The latter is important, because one of the aims of this thesis is to quantify droplet clustering in clouds by means of the radial distribution function, and the synthetic data should mirror this as closely as possible. 
Most KS are done on a non-periodic domain. The problem with that is that particles will bleed out of the region of interest, and over time the particle concentration decreases. This can be remedied in two ways: by seeding particles in a much larger region than the region of interest, or by using a domain with periodic boundary conditions. The downside of the former is that many more particles need to be simulated, than are within the region of interest at any given time. The downside of the latter is that the periodicity might be visible in the particle motions, especially on the large scale. Here both solutions are applied: the kinematic simulation is done on a cubic domain with sides $\mathcal{L}$ twice the size of the largest dimension of the particle tracking measurement volume, i.e. $\mathcal{L}=150 \mathrm{~mm}$.

\section{Velocity field}

The velocity field is composed of $N$ Fourier modes. Each of these is characterised by a wave vector $\boldsymbol{\kappa}_{n}$, frequency $\omega_{n}$, and Fourier coefficients $\boldsymbol{a}_{n}$ and $\boldsymbol{b}_{n}$. The velocity field is then given by

$$
\boldsymbol{u}(\boldsymbol{x}, t)=\sum_{n=1}^{N} \boldsymbol{a}_{n} \cos \left(\boldsymbol{\kappa}_{n} \cdot \boldsymbol{x}+\omega_{n} t\right)+\boldsymbol{b}_{n} \sin \left(\boldsymbol{\kappa}_{n} \cdot \boldsymbol{x}+\omega_{n} t\right) .
$$

The wave vectors and coefficients are chosen such that the resulting velocity field is triply periodic, incompressible, and exhibits a prescribed energy spectrum. Incompressibility $(\nabla \cdot \boldsymbol{u}=0)$ implies that

$$
\boldsymbol{a}_{n} \cdot \boldsymbol{\kappa}_{n}=0 \quad \text { and } \quad \boldsymbol{b}_{n} \cdot \boldsymbol{\kappa}_{n}=0
$$

For the velocity field to be periodic, it is convenient to first non-dimensionalise the spatial coordinates, such that they are on a cubic domain of size $(2 \pi)^{3}$, i.e. $\tilde{x} / 2 \pi=x / \mathcal{L}$. Then, the components of each wave vector $\kappa_{n}$ must be integers. A given energy spectrum $E(\kappa)$ finally gives the magnitudes of the Fourier coefficients:

$$
\left|\boldsymbol{a}_{n}\right|^{2}=\left|\boldsymbol{b}_{n}\right|^{2}=2 E\left(\kappa_{n}\right) \Delta \kappa_{n}
$$

with $\kappa=|\kappa| . \Delta \kappa_{n}$ is given by

$$
\Delta \kappa_{n}= \begin{cases}\frac{1}{2}\left(k_{2}-k_{1}\right) & \text { if } n=1 \\ \frac{1}{2}\left(k_{n+1}-k_{n-1}\right) & \text { if } 1<n<N \\ \frac{1}{2}\left(k_{N}-k_{N-1}\right) & \text { if } n=N\end{cases}
$$


For the energy spectrum the model spectrum by Pope [48] is used:

$$
\begin{gathered}
E(\kappa)=C \epsilon^{2 / 3} \mathcal{K}^{-5 / 3} f_{L}(\kappa L) f_{\eta}(\kappa \eta) \\
f_{\eta}(\kappa \eta)=\exp \left\{-\beta\left\{\left[(\kappa \eta)^{4}+c_{\eta}^{4}\right]^{1 / 4}-c_{\eta}\right\}\right\}
\end{gathered}
$$

with $C=1.5$ and $\beta=5.2$ empirically determined constants, and $c_{\eta}=0.40$ from turbulence theory. Here $f_{L}=1$, because $\mathcal{L}$ is much smaller than the large scales in atmospheric turbulence, and therefore wouldn't be visible anyway.

The parameters of the simulation are chosen as follows. Let $k_{c}$ be the largest wave number in the simulation.

1. Pick random wave vectors $\kappa_{n}$, such that they are on geometrically-spaced spherical shells in wave vector space, i.e. $\kappa_{n}=k_{c}^{(n-1) /(N-1)}$.

2. Round the components of each $k_{n}$ to the nearest integer.

3. As a result of the previous step, some wave vectors may end up having the same length, and equation (4.4) cannot directly be used to compute $\Delta \kappa_{n}$. To remedy this, $\Delta \kappa_{n}$ is computed using the first lower and first higher wave number. Example: if $\kappa=(1,1,2,3,4,4,5)$, then $\Delta \kappa=$ $\frac{1}{2}(1,1,2,2,2,2,1)$.

4. For each wave vector, count the total number of wave vectors that are in the same shell, and call this $m_{n}$. In the above example $m=$ $(2,2,1,1,2,2,1)$.

5. Pick random $\hat{\boldsymbol{a}}_{n}$ and $\hat{\boldsymbol{b}}_{n}$, compute such that $\hat{\boldsymbol{a}}_{n} \cdot k_{n}=0$ and $\hat{\boldsymbol{b}}_{n} \cdot k_{n}=0$, and they are of unit length.

6. Set the magnitudes of the $\boldsymbol{a}_{n}$ and $\boldsymbol{b}_{n}$ to $2 E\left(\kappa_{n}\right) \Delta \kappa / m_{n}$. The division by $m_{n}$ is to split the energy within a wave number shell over the (possibly) multiple wave vectors that are in it.

7. Set $\omega_{n}=\epsilon^{1 / 3} \kappa_{n}^{2 / 3}$ [22], and pick a random sign for each $\omega_{n}$.

A single set of parameters is used for the simulations performed here. These are summarised in table 4.1. The parameters are chosen such that the small scales are representative of cloud turbulence. The rms velocity and Taylor Reynolds number are much lower than in cloud turbulence, due to the absence of scales larger than $150 \mathrm{~mm}$. 
Table 4.1: Parameters used for the kinematic simulation. The rms velocity and Taylor Reynolds number are much lower than in cloud turbulence, due to the absence of scales larger than $150 \mathrm{~mm}$.

\begin{tabular}{lcc}
\hline Parameter & Symbol & Value \\
\hline Dissipation rate & $\epsilon$ & $0.024 \mathrm{~m}^{2} \mathrm{~s}^{-3}$ \\
Kinematic viscosity & $v$ & $1.82 \times 10^{-5} \mathrm{~m}^{2} \mathrm{~s}^{-1}$ \\
Kolmogorov length scale & $\eta$ & $0.69 \mathrm{~mm}$ \\
Kolmogorov time scale & $\tau_{\eta}$ & $28 \mathrm{~ms}^{-1}$ \\
RMS velocity & $u^{\prime}$ & $0.09 \mathrm{~m} \mathrm{~s}^{-1}$ \\
Taylor-Reynolds number & $\operatorname{Re}_{\lambda}$ & 50 \\
\hline Number of modes & $N$ & 108 \\
Cut-off wave number & $\kappa_{c}$ & $4524 \mathrm{~m}^{-1}$ \\
Integration time step & $\Delta t$ & $0.2 \mathrm{~ms}^{-}$ \\
\hline
\end{tabular}

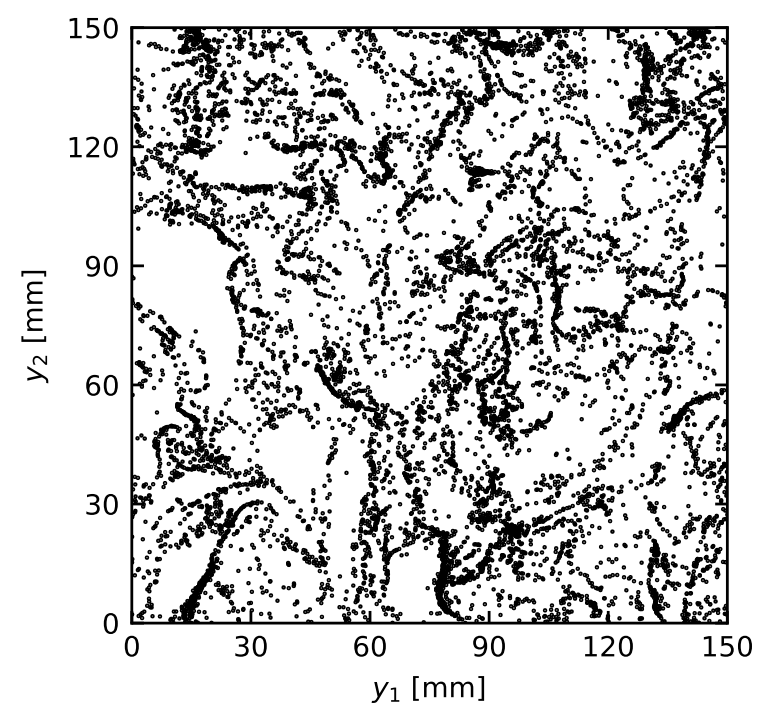

Figure 4.5: Particles in the kinematic simulation for $\mathrm{St}=1$. Shown is a $2 \mathrm{~mm}$ thick slice of the simulation domain. The particles are clearly clustering. 


\section{Particles}

Two cases are simulated: one with tracer particles $(S t=0)$ and one with strongly inertial particles $(\mathrm{St}=1)$. The inertial particles are only affected by Stokes drag, so their velocities can be computed with equation (2.21) with $g=0$. The particle positions and velocities (for $\mathrm{St} \neq 0$ ) are integrated using the Runge-Kutta 4 scheme. The time step is chosen to be equal to the frame time used for most experiments presented in this thesis, i.e. $0.2 \mathrm{~ms}$, which corresponds to a frame rate of $5 \mathrm{kHz}$. The highest expected number density $n$ in clouds at the Schneefernerhaus is $500 \mathrm{~cm}^{-3}$, so that $n \times \mathcal{L}^{3} \approx \sim 1.7 \times 10^{6}$ particles must be tracked. Figure 4.5 shows the particle positions within a $2 \mathrm{~mm}$ thick slice of the simulation domain. 


\section{Chapter 5}

\section{Droplet sizing}

\subsection{Introduction}

As discussed in chapter 2 the size of a droplet affects its behaviour in a turbulent flow both through the Stokes number St, which is a measure for its inertia, and through its settling velocity.

The aim of this chapter is to develop a method to measure the size of each droplet that the particle tracking experiment sees. The method should be implemented on top the existing setup, so all constraints that apply to that setup, also apply to the droplet sizing method. This means that there is only little space for extra light sources and/or cameras, and methods that can do without are preferred. One such method is an intensity-based method, i.e. droplet sizes are inferred only from the intensity of scattered light, which is already measured by the LPT experiment.

This chapter is organised as follows: first an overview of existing droplet sizing techniques is given. Then Lorentz-Mie scattering will be introduced and will be used to predict the intensity as observed by the LPT cameras (section 5.3. After that a droplet-sizing method will be proposed and calibrated (sections 5.4 and 5.5). The method is finally applied to field data and compared to droplet sizes obtained using a holographic instrument (section 5.6).

\subsection{Techniques}

A short review of existing particle sizing techniques is given here. For each technique its applicability to the Zugspitse experiment is assessed. 
Optical particle counters (OPCs) [23, 28, 31, 32, 35] These devices illuminate a small measurement volume using white light or a laser beam, and collect scattered light with large lenses or curved mirrors, which is then directed onto one or more photodetectors. The particle or droplet size is calculated using only the measured light intensity. The collection angle is typically quite large: between $20^{\circ}$ and $120^{\circ}$ degrees. Such a large collection angle has two effects: first, it allows these devices to collect as much light as possible, so that even particles of only $0.1 \mu \mathrm{m}$ in diameter can be measured. Second, a large collection angle helps averaging over the oscillations that are typical for Lorentz-Mie scattering.

Altough OPCs perform single-point measurements, the technique can be implemented in higher dimensions using cameras. Within the Zugspitze project this can be done with the existing particle tracking setup, so no extra cameras are needed, but the oscillations in the Lorentz-Mie scattering would need to be dealt with somehow.

Phase Doppler anemometry (PDA) [42] In PDA two coherent laser beams are used to illuminate a small measurement volume from two slightly different directions, and scattered light is collected using a number of photodetectors that are set up around the measurement volume. When a droplet moves through the measurement volume, it will show two glare points, one per laser beam, due to refraction of each beam. The light of the two glare points interferes and creates a fringe pattern in the far field, the angular frequency of which is proportional to the droplet's size. As the droplet moves through the measurement volume, the fringe pattern moves with it, and is recorded by the photodetectors as a Doppler burst. The phase difference between the bursts as measured by the various detectors is proportional to the droplet's size.

PDA is a single-point measurement technique, and is therefore not suitable for application at Zugspitze. It has been extended to multiple dimensions, using laser sheets, which is described next.

Global phase Doppler (GPD) [13] GPD is an extension of PDA to multiple dimensions. It uses two coplanar, coherent laser sheets to illuminate a relatively large measurement volume. Each droplet's fringe pattern is recorded using a defocused camera.

Interferometric particle imaging (IPI) [7, 13] IPI is similar to GPD, but uses only a single laser sheet. The two glare points that are needed to create the 
fringe pattern, are not due to refraction of two laser sheets, but due to refraction and reflection of a single laser sheet. The two glare points should be of similar intensity for IPI to work, but since refraction and reflection respond differently to variation of the scattering angle, this puts constraints on where the camera can be placed.

Using IPI or GPD with the Zuspitze experiment would require an extra camera, which is undesirable. Furthermore, due to the high number densities in clouds, the defocused particle images would dramatically overlap, which makes the analysis difficult if not impossible.

Holography [42, 59] Schlenczek [59] summarises holography well: "A hologram is made when a reference wave interferes with an object wave and the interference pattern is captured on film (analog) or on an image sensor (digital)." In holography one does not capture an image of an object, but the wavefront as it is scattered by that object. The recorded wavefront can later be used to reconstruct the wavefront at various distances from the sensor. By reconstructing the wavefront at the position of a droplet, the droplet's size can be determined.

To implement holography within the Zugspitze experiment, an extra laser and an extra camera are needed, which is undesirable.

Shadowgraphy [36] In shadowgraphy a collection of droplets it backlit by a diffuse light source. A camera is aimed directly at the light source, so that the droplets cast shadows onto the camera sensor. The size of droplets that are in focus, can be determined by simply counting pixels. Methods exist to get reliable size information for defocused droplets as well.

To implement shadowgraphy within the Zugspitze experiment, an extra laser and an extra camera are needed, which is undesirable.

\subsection{Theory}

\subsubsection{Lorentz-Mie scattering}

This section is based on Bohren and Huffman [9].

The scattering of electromagnetic waves, such as light, by a sphere is described by the Lorentz-Mie equations. The equations are summarised here; for a derivation the reader is referred to e.g. Bohren and Huffman [9]. 


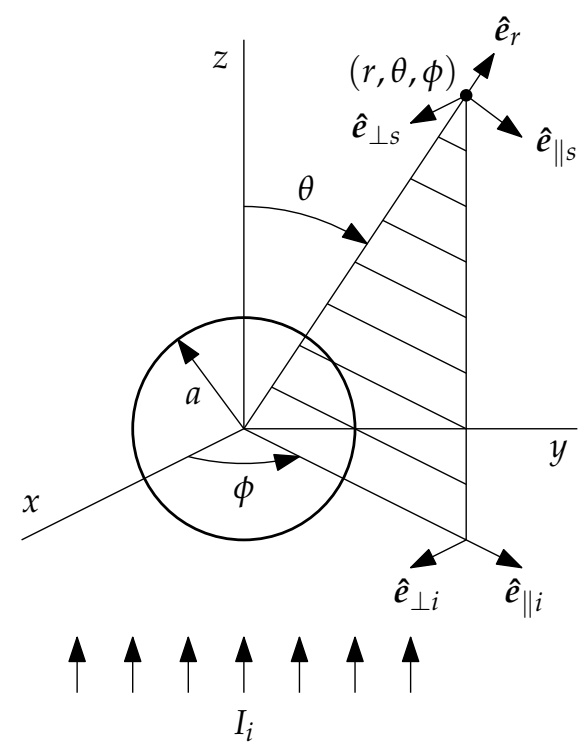

Figure 5.1: An EM wave travelling in the $+z$ direction is incident upon a sphere with radius $a$. The scattered fields are computed at $r=(r, \theta, \phi)$. The scattering plane is determined by $\hat{\boldsymbol{e}}_{z}$ and $\hat{\boldsymbol{e}}_{x}$. The three unit vectors $\hat{\boldsymbol{e}}_{z}, \hat{\boldsymbol{e}}_{\| i}$, and $\hat{\boldsymbol{e}}_{\perp i}$ form an orthogonal basis. The three unit vectors $\hat{\boldsymbol{e}}_{r}, \hat{\boldsymbol{e}}_{\| s}$, and $\hat{\boldsymbol{e}}_{\perp s}$ form an orthogonal basis as well. Unit vectors with subscript $\|$ are parallel to the scattering plane; unit vectors with subscript $\perp$ are perpendicular to the scattering plane. 
Consider the system shown in figure 5.1. A sphere is of radius $a$ is located at the origin. It has a complex index of refraction $N_{1}$, while the medium around it has a real index of refraction $N$; the relative refractive index is $m=N_{1} / N$. The imaginary part of $N_{1}$ (and hence of $m$ ) can be nonzero, which means that the sphere's material is absorbing at that wavelength. A plane EM wave with wave number $k$ in the medium is travelling in the $+z$ direction, and is incident upon the sphere. The size parameter $x$ is given by $k a$. The aim is to compute the irradiance $I_{s}$ of the scattered radiation at a point $(r, \theta, \phi)$, given the irradiance $I_{i}$ of the incident light. The vectors $\hat{\boldsymbol{e}}_{z}$ and $r$ define the scattering plane, which in turn is used to define the various unit vectors parallel and perpendicular to it. In the following only the electric field will be considered. The corresponding magnetic field can be obtained through the Maxwell equations, and given both fields the irradiance can be computed through the Poynting vector.

The electric field part of the incident wave can be split in two components,

$$
\boldsymbol{E}_{i}=\left(E_{\| i} \hat{\boldsymbol{e}}_{\| i}+E_{\perp i} \hat{\boldsymbol{e}}_{\perp i}\right),
$$

one parallel to the scattering plane and one perpendicular to it. Far away from the droplet the scattered field can be decomposed similarly:

$$
\boldsymbol{E}_{S}=\left(E_{\| s} \hat{\boldsymbol{e}}_{\| S}+E_{\perp s} \hat{\boldsymbol{e}}_{\perp S}\right) .
$$

The relation between the incident field and the scattered field can then be written as

$$
\left[\begin{array}{c}
E_{\| s} \\
E_{\perp s}
\end{array}\right]=\frac{e^{i k(r-z)}}{-i k r}\left[\begin{array}{ll}
S_{2} & S_{3} \\
S_{4} & S_{1}
\end{array}\right]\left[\begin{array}{c}
E_{\| i} \\
E_{\perp i}
\end{array}\right],
$$

with $S_{i}$ the elements of the amplitude scattering matrix. For scattering by a sphere $S_{3}=S_{4}=0$, and

$$
\begin{aligned}
& S_{1}=\sum_{n} \frac{2 n+1}{n(n+1)}\left(a_{n} \pi_{n}+b_{n} \tau_{n}\right), \\
& S_{2}=\sum_{n} \frac{2 n+1}{n(n+1)}\left(a_{n} \tau_{n}+b_{n} \pi_{n}\right),
\end{aligned}
$$

with $\pi_{n}$ and $\tau_{n}$ defined as

$$
\begin{aligned}
\pi_{n} & =\frac{P_{n}^{1}(\cos \theta)}{\sin \theta}, \\
\tau_{n} & =\frac{\mathrm{d} P_{n}^{1}(\cos \theta)}{\mathrm{d} \theta},
\end{aligned}
$$


with $P_{n}^{1}$ the first derivative of the Legendre polynomial of order $n . a_{n}$ and $b_{n}$ are the scattering coefficients, and are given by

$$
\begin{aligned}
& a_{n}=\frac{m \psi_{n}(m x) \psi_{n}^{\prime}(x)-\psi_{n}(x) \psi_{n}^{\prime}(m x)}{m \psi_{n}(m x) \xi_{n}^{\prime}(x)-\xi_{n}(x) \psi_{n}^{\prime}(m x)}, \\
& b_{n}=\frac{\psi_{n}(m x) \psi_{n}^{\prime}(x)-m \psi_{n}(x) \psi_{n}^{\prime}(m x)}{\psi_{n}(m x) \xi_{n}^{\prime}(x)-m \xi_{n}(x) \psi_{n}^{\prime}(m x)},
\end{aligned}
$$

with the Riccati-Bessel functions $\psi_{n}(\rho)=\rho j_{n}(\rho)$ and $\xi_{n}(\rho)=\rho h_{n}^{(1)}(\rho)$, where $j_{n}$ is the spherical Bessel function and $h_{n}^{(1)}$ is the spherical Hankel function (both of the first kind), and a prime indicating differentiation.

In practice the complex scattering amplitudes $S_{1}, \ldots, S_{4}$ are difficult to work with, because they related not only the magnitude, but also the phase of the scattered field to the incident field, and are therefore difficult to measure. Instead the Stokes parameters $I, Q, U$, and $V$ are used. These parameters are defined in terms of how they are measured, which is by putting various polarisers in front of a detector that by itself measures only irradiance. In particular:

- $I$ is measured without any polarisers;

- $Q$ is the difference between the irradiances measured with a horizontal polariser, and with vertical polariser;

- $U$ is as $Q$, but with $45^{\circ}$ and $-45^{\circ}$ polarisers; and

- $V$ is as $Q$, but with left-handed and right-handed circular polarisers.

For a scattering by a sphere, the Stokes parameters of the scattered field can be related to those of the incident field as follows:

$$
\left[\begin{array}{c}
I_{s} \\
Q_{s} \\
U_{s} \\
V_{s}
\end{array}\right]=\frac{1}{k^{2} r^{2}}\left[\begin{array}{cccc}
S_{11} & S_{12} & 0 & 0 \\
S_{12} & S_{11} & 0 & 0 \\
0 & 0 & S_{33} & S_{34} \\
0 & 0 & -S_{34} & S_{33}
\end{array}\right]\left[\begin{array}{c}
I_{i} \\
Q_{i} \\
U_{i} \\
V_{i}
\end{array}\right],
$$

with the scattering matrix elements

$$
\begin{aligned}
& S_{11}=\frac{1}{2}\left(\left|S_{2}\right|^{2}+\left|S_{1}\right|^{2}\right), \\
& S_{12}=\frac{1}{2}\left(\left|S_{2}\right|^{2}-\left|S_{1}\right|^{2}\right), \\
& S_{33}=\frac{1}{2}\left(S_{2}^{*} S_{1}+S_{2} S_{1}^{*}\right), \\
& S_{34}=\frac{i}{2}\left(S_{2}^{*} S_{1}-S_{2} S_{1}^{*}\right) .
\end{aligned}
$$


Unpolarised light has $Q=U=V=0$, so if that hits a droplet, the scattered irradiance is given by

$$
I_{S}=\frac{1}{k^{2} r^{2}} S_{11}\left(\theta^{\prime}\right) I_{i}
$$

Various codes have been written to compute the scattering matrix elements $S_{i j}$ for a spherical particle numerically [19]. Here the popular BHMIE code [10] is used to compute scattering matrix element $S_{11}$, which represents scattering of unpolarised light, for various droplet diameters and scattering angles. The result is plotted in figure 5.2 As can be seen in the top graph, $S_{11}$ grows proportional to $d_{p}^{2}$, but also oscillates strongly and has a fine structure. The intensity ratio between peaks and valleys is about 2.5 , as can be estimated from the inset. The most important thing to note in the bottom graph, is that even though in general $S_{11}$ is a very irregular function of $\theta$, in the range from $25^{\circ}$ to $35^{\circ}$ it is slightly better behaved: it decreases more or less exponentially, and "only" oscillates strongly.

\subsubsection{Integration of scattered intensity}

The theory presented in the previous section provides a way to compute the scattered irradiance at a single point in space. In practice the camera objectives have an aperture with a finite diameter, over which the scattered irradiance must be integrated. This will prove to be beneficial, because to some extent it helps averaging out the oscillations in the Lorentz-Mie scattering patterns. In fact, the oscillations can be suppressed even further by putting a diffuser in the laser beam, as will be shown here.

The scattered intensity $I_{O}$ that a camera would observe is given by the following equation:

$$
I_{O}=\iint_{A} \mathrm{~d} A\left(x_{a}^{\prime}\right) \iint_{4 \pi} w_{d} \mathrm{~d} \Omega\left(\hat{l}^{\prime}\right) I_{s}\left(r^{\prime}, \theta^{\prime}\right)
$$

The first integral is over the aperture $A$. The second is over a sphere, but is weighted by $w_{d}$, a two-dimensional Gaussian that represents the diffuser's angular spread. Lastly, $\theta^{\prime}$ is the local scattering angle, which depends on the droplet position, the position $x_{p}^{\prime}$ of the aperture surface infinitesimal, and the local laser direction $\hat{l}^{\prime}$.

Equation (5.4) could be evaluated directly, but since it contains two twodimensional integrals this would be rather time-consuming, even more so if one considers that it should be done for many droplet sizes and many 

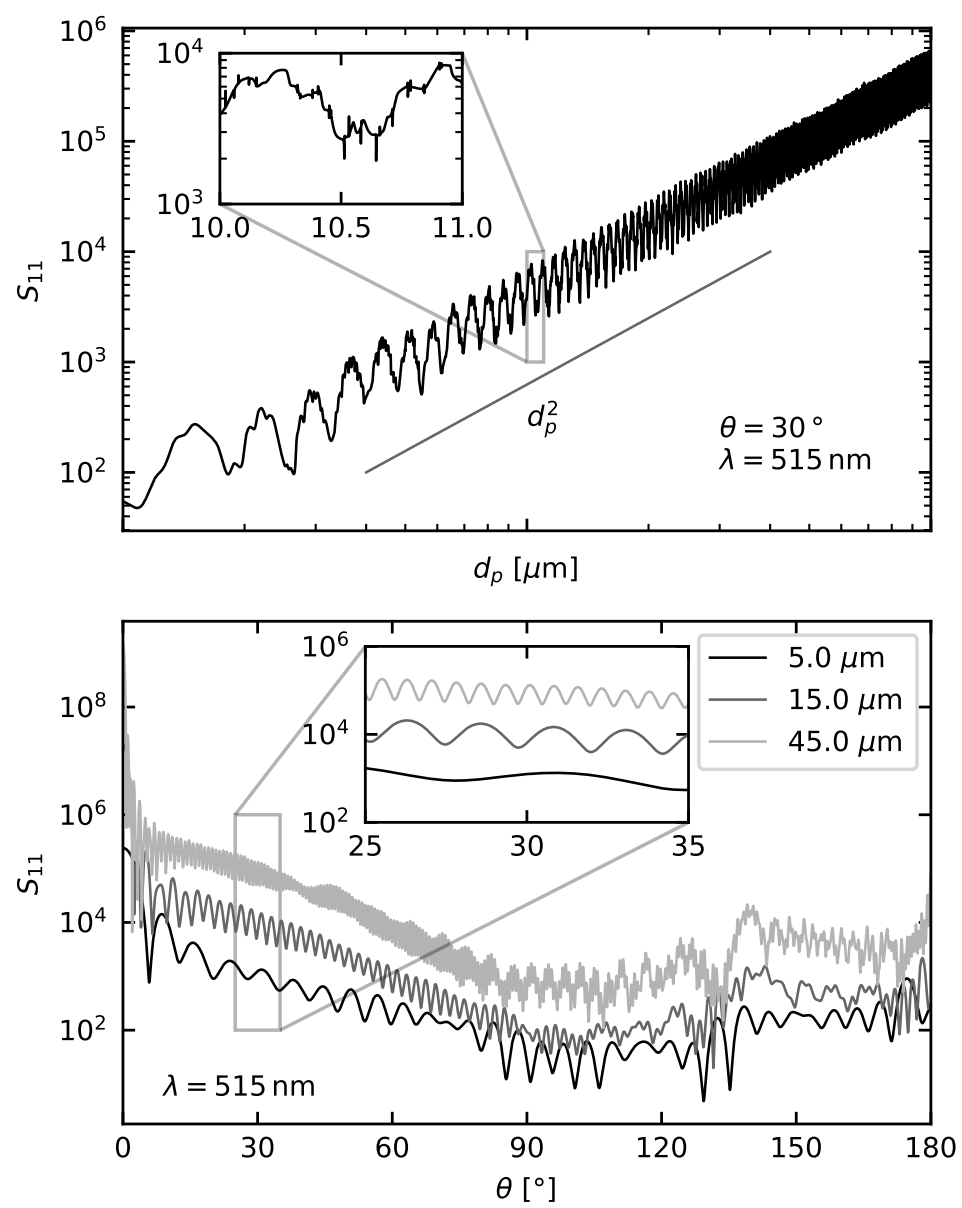

Figure 5.2: Scattering matrix element $S_{11}$, which represents scattering of unpolarised light, as a function of droplet diameter (top), and as a function of scattering angle (bottom). 
scattering angles. However, if the aperture subtends a sufficiently small angle, the FWHM of the diffuser is sufficient small, and the scattering angle is large enough, then the dimension of the double integrals that is perpendicular to the scattering plane can be evaluated immediately, yielding two single integrals. After a change of variables one of these integrals can again be evaluated immediately, which gives the following:

$$
I_{O}=\int_{-\infty}^{\infty} \mathrm{d} \xi I_{S}(\theta+\xi) h(\xi) .
$$

with $\theta$ the central scattering angle, that is the angle between the dropletaperture vector $r$ and the laser beam's axis $\hat{l}$, and $h$ a function that represents the geometry of the droplet, the aperture and the diffuser. The full derivation can be found in appendix A

The apertures of the objectives used in the LPT setup subtend angles of $1^{\circ}$, the beam expander is equipped with a $1^{\circ}$ FWHM diffuser, and the central scattering angle varies between $25^{\circ}$ and $35^{\circ}$. Given these numbers, equation (5.5) can safely be used.

To see what the effect of the aperture and the diffuser on the observed intensity is, equation (5.5) is evaluated for many droplet sizes, both without and with the diffuser, and is compared with Lorentz-Mie scattering cf. equation (5.3). The result is shown in figure 5.3. As can be seen, the aperture by itself reduces the oscillations to some extent: they practically disappear at $55 \mu \mathrm{m}$, but come back for larger sizes, although there they are not as strong. Adding the diffuser reduces the oscillations even further: they disappear at $35 \mu \mathrm{m}$, and do not return. It is likely that the diffuser is more effective at reducing oscillations than the aperture is, because the diffuser has smooth edges due to its Gaussian character, whereas the aperture has sharp edges.

So, for large droplets it should be possible to compute their size from their observed intensity as $d_{p} \propto \sqrt{I_{O}}$.

In figure 5.4 the same quantities are plotted, but now as a function of (central) scattering angle $\theta$. As can be seen the aperture and diffuser together are very effective at averaging out the oscillation for droplets of $30 \mu \mathrm{m}$. Also shown is a exponential fit of the observed intensity with diffuser, $I_{O} \sim$ $\exp \left(\theta / c_{\theta}\right)$, with $c_{\theta}=-15.7^{\circ}$.

\subsection{Method}

The theory presented in previous section should now be turned into a droplet sizing method. For droplets larger than $35 \mathrm{~mm}$ this is simple: $d_{p}=c_{0} \sqrt{I}$, with 

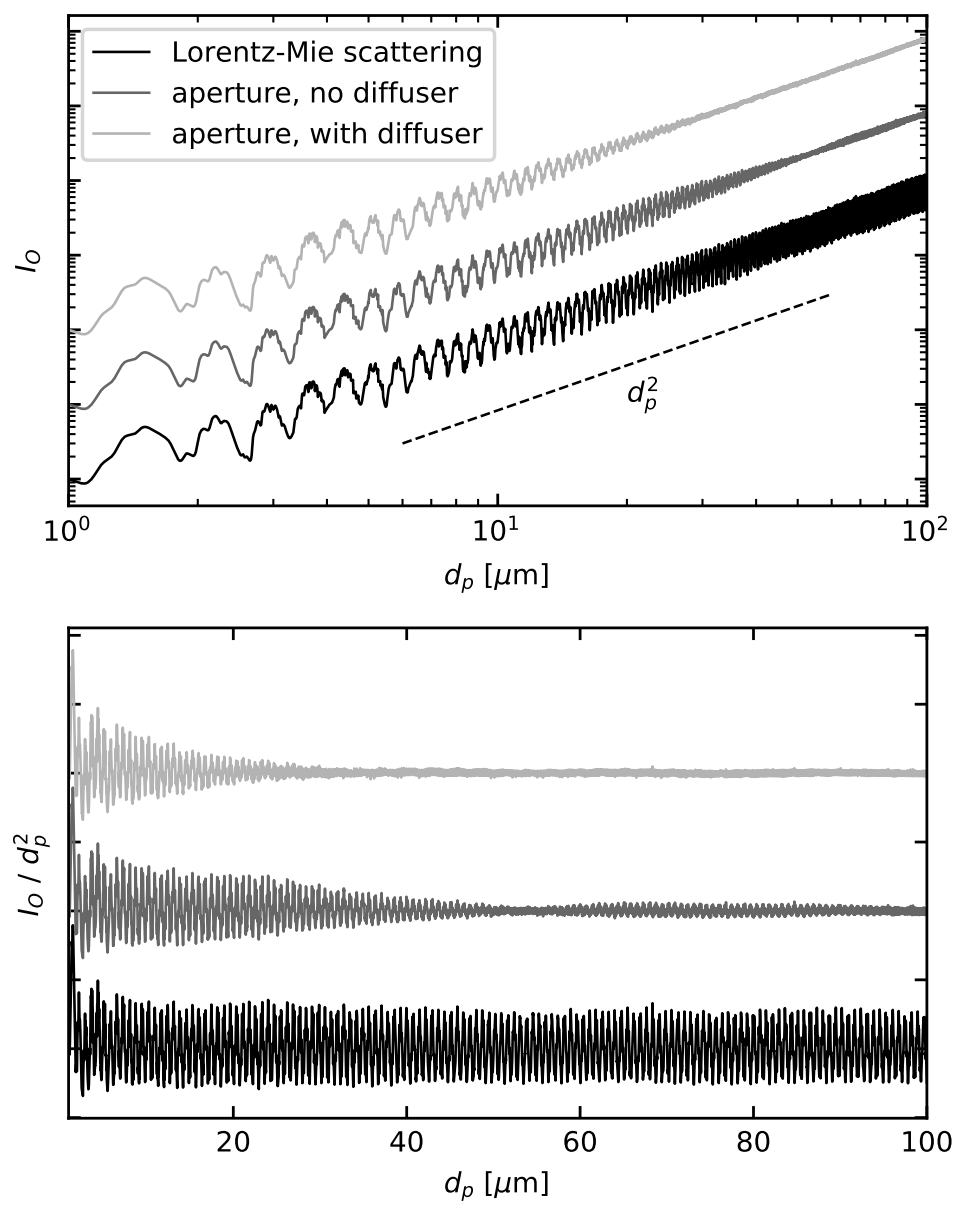

Figure 5.3: Comparison of the observed intensity $I_{O}$, both without and with diffuser, with Lorentz-Mie scattering. The observed intensity is calculated by integrating Lorentz-Mie scattering over the diffuser and over the camera's aperture. The top and bottom graphs shows the same data, but on different scales, and with different normalisation. The curves are have been offset, so they do not overlap. The scattering angle is $30^{\circ}$. 

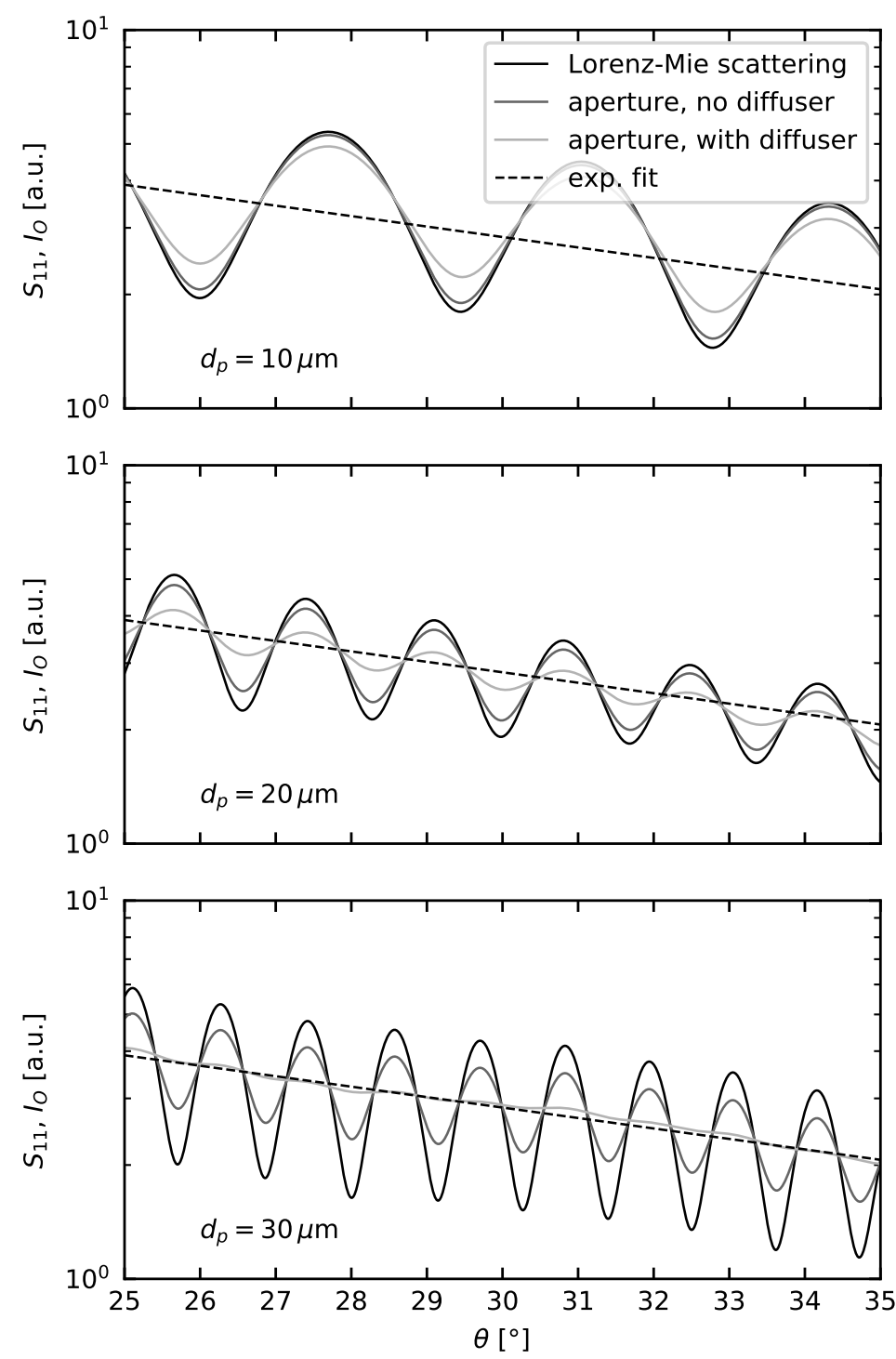

Figure 5.4: Comparison of the observed intensity $I_{O}$, both without and with diffuser, with Lorentz-Mie scattering. The observed intensity is calculated by integrating Lorentz-Mie scattering over the diffuser and over the camera's aperture. Also shown is an exponential function $\exp \left(\theta / c_{\theta}\right)$, with $c_{\theta}=-15.7^{\circ}$, that was fitted to $I_{O}$ for the case with diffuser. 
$c_{0}$ some calibration constant. This is simple and fast, and is therefore attractive to also apply to the smaller droplets. But for this to work for smaller droplets, the remaining Lorentz-Mie oscillations need to be dealt with. Furthermore a scheme is needed combine all of a droplet's observed intensities $I_{i j}$, with $i$ a point along a droplet's trajectory and $j$ a camera, into a single intensity.

For the LPT setup presented in the previous chapter both problems can be solved by averaging over all of a droplet's observed intensities $I_{i j}$. Of course averaging only makes sense if these intensities come from the same distribution, which techically speaking they do not. However, given that (1) all camera views are under similar angles to the laser beam's axis, (2) all apertures are of similar size, and (3) all objectives are similarly far away from the origin, it can be argued that the observed intensities come from distributions that are at least similar to each other. The distributions can be made more similar by applying the following corrections, each of which corrects for changes that result from changes in a droplet's positions:

Droplet-aperture distance. If the droplet-aperture distance $r$ changes, then this effectively changes the aperture's subtended solid angle, and hence the part of the Lorentz-Mie scattering pattern that is integrated over. The subtended solid angle is proportional to $r^{2}$, so this can be corrected for by dividing by $\left.\left(r / r_{0}\right)^{2}\right)$, with $r_{0}$ some reference distance.

Scattering angle. In the previous section it was shown that the intensity scales with an exponential function, $\exp \left(\theta / c_{\theta}\right)$. To correct for changes in scattering angle, the intensity can be divided by $\exp \left[\left(\theta-\theta_{0}\right) / c_{\theta}\right]$, with $\theta_{0}$ some reference scattering angle.

Laser beam intensity. The profile of the laser beam is known (see chapter 4), and equation (5.5) is linear in the intensity of the incident light $I_{i}$, so given the droplet's position, the local laser beam intensity can simply be divided out.

The corrections for droplet-aperture distance and scattering angle can also be seen as a first-order expansion of the integrals in equation 5.4 around some reference droplet position $x_{p}$.

Averaging a droplet's observed intensity over the three cameras, and over all points in a trajectory, is not just valid, but it even helps to reduce LorentzMie oscillations. Because the camera views are under similar, but slightly different angles, averaging the intensitites over the three cameras effectively increases the aperture size and hence reduces Lorentz-Mie oscillations. Furthermore, as a droplet moves through the measurement volume, the scattering 
angle $\theta$ also changes, which again helps to average out the Lorentz-Mie oscillations. As a reminder, the camera view angles $\theta_{0}$ are $28.1^{\circ}, 30.5^{\circ}$, and $33.0^{\circ}$ (see chapter 3).

Considering all of the above, the following droplet-sizing method is proposed:

Given a droplet's observed intensities $I_{i j}$, with $i$ a point along the droplet's trajectory, and $j$ a camera, do the following:

1. Apply the corrections for droplet-aperture distance, scattering angle, and laser beam intensity, as described above. This yields the corrected intensities $I_{i j, c}$.

2. Compute the average intensity $I=\left\langle I_{i j, c}\right\rangle$.

3. Compute the droplet size $d_{p}=c_{0} \sqrt{I}$, with $c_{0}$ a calibration constant.

The droplet size uncertainty is computed by first computing the intensity variance $\sigma_{I}^{2}$, and then the relative droplet size uncertainty

$$
\frac{\sigma_{d_{p}}}{d_{p}}=\frac{1}{2} \frac{\sigma_{I}}{I},
$$

which is obtained using standard propagation of uncertainty.

Interally the various codes use the logarithm of the intensity, $\ln \left(I_{i j}\right)$, so instead of computing arithmetic means, in practice geometric means are computed. The droplet size uncertainty is computed differently, too:

$$
\frac{\sigma_{d_{p}}}{d_{p}}=\sinh \left(\frac{1}{2} \sigma_{I}\right),
$$

with $\sigma_{I}$ the logarithm of the geometric standard deviation.

One could argue that instead of the sample variance $\sigma_{I}^{2}$, the variance $\sigma_{I}^{2} / N$ of the mean, with $N$ the number of points in the track, should be used to compute the droplet size uncertainty. That isn't done here, because for small particle sizes the oscillations in the predicted intensity $I_{O}$ still cause ambiguity that isn't dealt with. Hence, while for larger droplets using the sample variance leads to an overestimate of the uncertainty, using the variance of the mean would lead to an underestimate, which arguably is a worse mistake to make. 


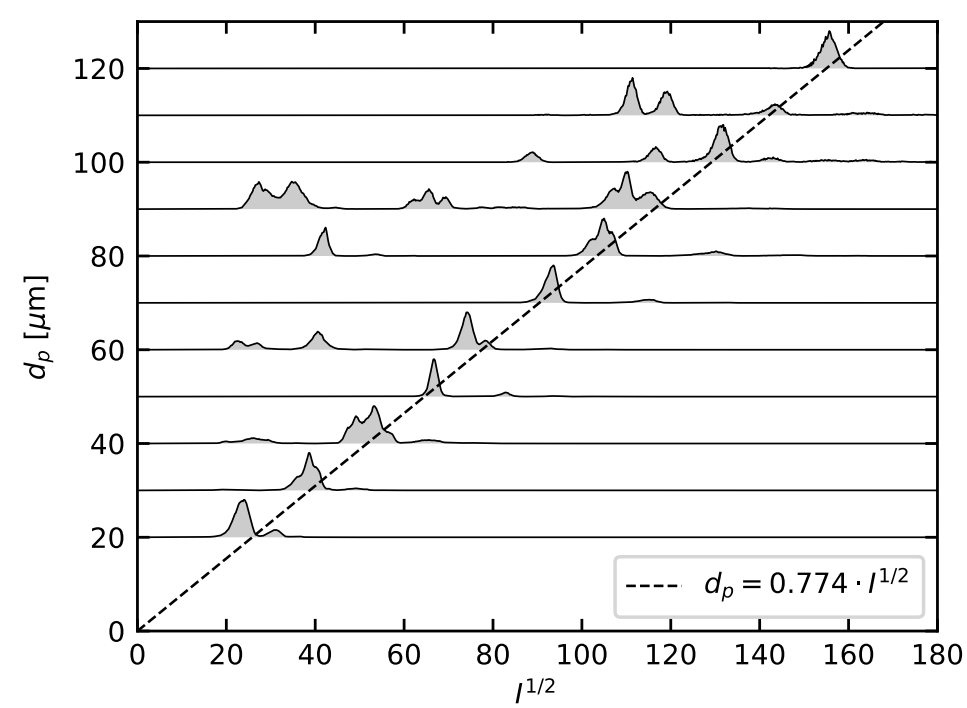

Figure 5.5: Histrograms of square-root-droplet-intensity $\sqrt{I}$ for droplets produced with a calibrated aerosol generator. For each target diameter that the aerosol generator was set up for, a corresponding histogram is shown in here. Each histogram has been offset vertically by its corresponding target droplet diameter. The dashed line is $d_{p}=c_{0} \sqrt{I}$, with the calibration constant $c_{0}=0.774$.

\subsection{Calibration}

Before the droplet-sizing method can be applied to data from field experiments, the calibration constant $c_{0}$ must be determined. To do this, a number of LPT experiments is performed in the laboratory, using droplets generated with a calibrated aerosol generator. Droplets are used instead of glass beads to avoid any problems that could arise due to the sensitivity of Lorentz-Mie scattering to non-sphericity and changes in the refractive index. The droplets are generated with a TSI FMAG Model 1520, a commercial aerosol generator, that is calibrated using a shadowgraphy experiment (see appendix C).

The aerosol generator is set up to generate droplet diameters $d_{p}=20,30$, $\ldots, 120 \mu \mathrm{m}$. For each droplet diameter, the following steps are taken:

1. Particle videos are recorded at different laser powers, and are analysed 
using $\mathrm{L}^{3} \mathrm{PT}$.

2. For each droplet the average intensity $I$ is computed as per the previous section.

3. A histogram of $\sqrt{I}$ is made.

The histograms are plotted in figure 5.5. They are offset by their corresponding droplet diameters, so that the peaks' bases should lie on a line, which they indeed do. The slope of this line is the calibration constant $c_{0}$, and was found to be 0.774 , which was determined using only the location of the peak value of the $120 \mu \mathrm{m}$ histogram.

During the experiments it was noticed that the aerosol generator sometimes produced a secondary jet. This suggests that it was not correctly set up, which could explain the extra peaks in the histograms.

\subsection{Results}

The droplet sizing method is applied to data collected at the Zugspitze on 25-9-2020. To get some sense for its performance, droplet size PDFs are compared with (preliminary) droplet size PDFs obtained using the HALOHolo holography instrument [59]. The HALOHolo was installed a few centimetres downwind of the LPT measurement volume, to ensure that it would measure the same flow as the LPT experiment did. Droplet size PDFs for various half-hour-long periods are shown in figure 5.6 As can be seen they follow the same trends, but do not agree completely. The latter could be remediated by doing a more careful analysis of the HALOHolo data.

One noteworthy aspect of the droplet size PDFs obtained with the intensity-based method, are the oscillations for small droplet sizes, i.e. $d_{p} \lesssim 15 \mu \mathrm{m}$. These are most likely the result of the remaining oscillations in observed intensity, as is shown in figure 5.3

Cumulative distributions of droplet size and relative uncertainty $\sigma_{d_{p}} / d_{p}$ are shown in figure 5.7 Note that these distributions show $P\left(\sigma_{d_{p}} / d_{p}>X\right)$, i.e. the probability that the relative uncertainty is larger than a certain value, and therefore decrease instead of increase. In general, the relative uncertainty decreases with increasing drop size. The median relative uncertainty is approximately 0.05 . For droplets larger than $10 \mu \mathrm{m}$ in diameter, the relative uncertainty tends to exceed 0.1 in no more than $1 \%$ of cases. 

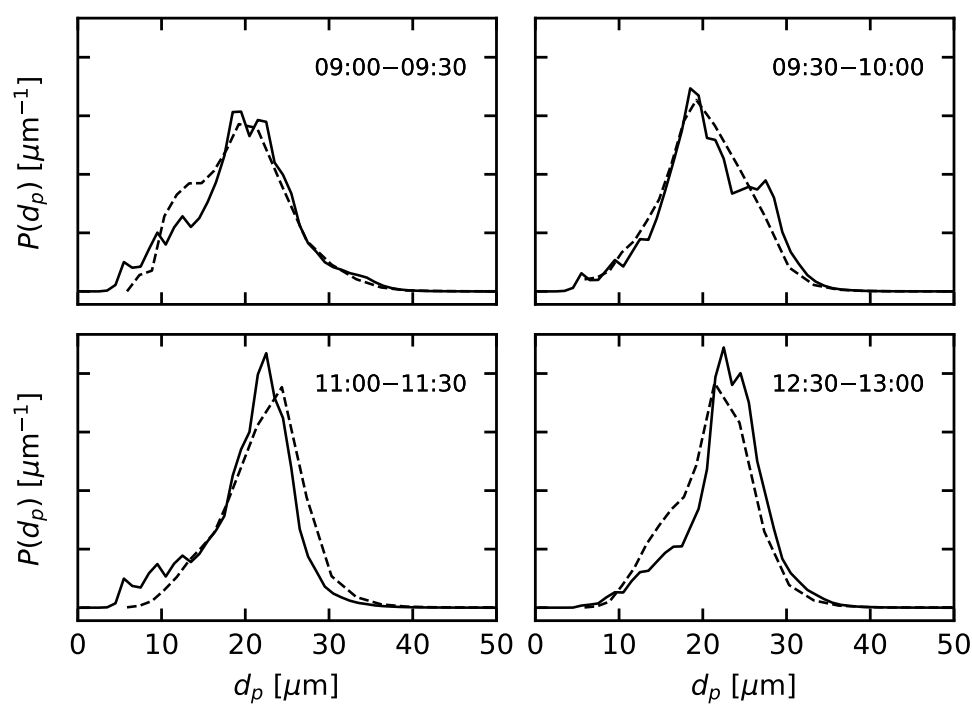

Figure 5.6: Droplet size PDFs inferred with the intensity-based method (-), and as measured by the HALOHolo (- -). The data were collected at the Zugspitze during various half-hour-long periods on 25-9-2019. Times are UTC. The HALOHolo data shown here are the result of a preliminary analysis. 


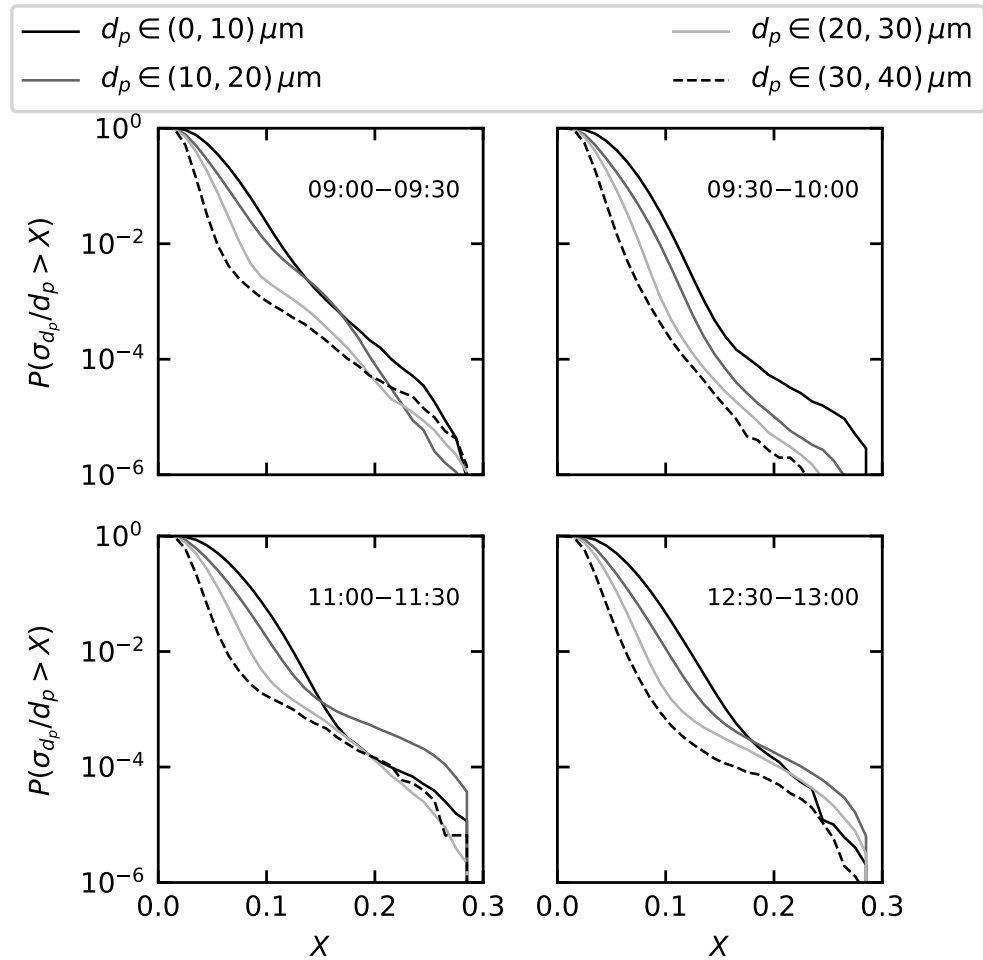

Figure 5.7: CDFs of relative uncertainty for various ranges of droplet sizes. The data were collected at the Zugspitze during various half-hour-long periods on 25-9-2019. Times are UTC. Note that these are reversed CDFs, i.e. the quantity shown is the probability that the uncertainty is larger than a certain value. 


\subsection{Conclusion}

Using Lorentz-Mie theory the intensity scattered by a droplet into a camera is predicted, taking both the camera's aperture and the diffusivity of the light source into account. It is shown that the camera's aperture averages out the oscillations in the scattering pattern to some extent, and that the addition of a diffuser helps to further reduce the oscillations.

For the Zugspitze experiment a diffuser with a diffusion angle of $1^{\circ} \mathrm{FWHM}$ is chosen, because larger angles would negatively affect the particle tracking performance, or would require a redesign the laser beam expander. With this diffuser the oscillations in the scattering pattern disappear for droplet diameters $>35 \mu \mathrm{m}$. When a droplet moves through the measurement volume the scattering angle changes, so by averaging a droplet's observed intensity over its trajectory, the remaining oscillations in the scattering pattern can be averaged out to some extent.

The proposed droplet sizing method works as follows: first, the measured intensities are corrected for droplet-aperture distance and scattering angle. Then the average intensity $I$ of the corrected intensities is computed. Finally the droplet size is computed as $d_{p}=c_{0} \sqrt{I}$, with $c_{0}$ a calibration constant that is determined in the laboratory.

The method is applied to data collected with the particle tracking setup discussed in the previous chapter, and is compared with data from the HALOHolo. Based on the results shown here, it is fairly safe to say that the method works, but to gain more confidence in the method, it must be compared with HALOHolo data taken on different days. The median relative uncertainty is $\sim 5 \%$, which should be sufficient for analysing cloud droplet behaviour.

\subsection{Outlook}

For application at Zugspitze the droplet sizing method would probably benefit from an even larger diffusion angle. The theory developed in section $5.3 \mathrm{can}$ be used see if this indeed has the desired effect. If this is the case, the beam expander will need to be redesigned using a larger lens at the exit.

If the particle tracking post-processing code is extended to compute the droplet number density, absolute droplet size spectra (unit: $1 / \mu \mathrm{m} / \mathrm{cm}^{3}$ ) could be computed, which could then be compared with other instruments. This would help to verify the droplet sizing method, which in turn could be used to asses the performance of the particle tracking setup for various droplet sizes.

To make this chapter suitable for publication, the effect of having multiple 
apertures at slightly different scattering angles should be analysed thoroughly. Furthermore it would be useful to extend the theory to white light. Finally the droplet sizing method should be demonstrated to work reliably for a range of smaller droplet sizes, e.g. from $5 \mu \mathrm{m}$ to $30 \mu \mathrm{m}$.

Preliminary work was done to develop a method that directly uses the scattered intensity $I_{O}$ to perform Bayesian inference on observed intensities. Although this method has the potential to give more accurate droplet sizes for small droplets, it is much more sensitive to the exact geometry of the experiment, and is also very slow. This work is documented in appendix B 


\section{Chapter 6}

\section{Turbulence statistics}

In all turbulence experiments some basic flow parameters must be known. These are, for example, the mean velocity $U$ (if present), the rms velocity $u^{\prime}$, and the dissipation rate $\epsilon$. For the experiments presented in this thesis, the dissipation rate is of key importance, because it directly influences the cloud droplets' Stokes numbers.

In laboratory experiments these quantities are typically measured using either of three techniques: hot-wire anemometry, PIV, or PTV. However non of these is suitable for measuring atmospheric flows. Hot-wire probes are delicate devices, that can easily break if a too large particle (e.g. sand) is blown into it. PIV is rather cumbersome, in that it requires a second weather-proof optical setup. The LPT experiment that is at the heart of this thesis can't be used either: cloud droplets are inertial particles, i.e. they aren't tracer particles, and hence don't probe the Kolmorogorov scales reliably. Furthermore a typical experiment lasts for only $3.3 \mathrm{~s}$, which is too short to sufficiently probe the inertial subrange.

Atmospheric flows are typically measured with sonic anemometers 64 ch. 17]. These instruments put two ultrasonic transducers opposite each other, typically separated by $5 \mathrm{~cm}$ to $20 \mathrm{~cm}$. By sending ultrasound pulses between the two transducers, and measuring the travel time in each direction, the velocity of the medium through which the pulses propagate can be determined. In this work a 3D sonic is used: it has three pairs of transducers, so that it can measure all three components of the velocity.

A sonic anemometer measures the flow velocity $\boldsymbol{u}(t)$ at a single point in space, as a function of time $t$. Turbulence, however, is described in terms of the velocity field, i.e. $\boldsymbol{u}(\boldsymbol{x})$ at a single time, as a function of spatial coordinate 
$x$. To get spatial data from the sonic anemometer, Taylor's frozen turbulence approximation [63] is used. This approximation assumes that the velocity field is frozen in space, and is merely carried past the sonic by some mean velocity $U$, so that $\boldsymbol{u}(x)=\boldsymbol{u}(\boldsymbol{U} t)$.

In reality the velocity field is not frozen; larger scales convect the smaller scales with the rms velocity $u^{\prime}$ [39], so for Taylor's approximation to work, the turbulent intensity $I=u^{\prime} / U$ should be sufficiently small. The interpretation of "sufficiently small" varies between publications: many articles refer to Willis and Deardorff [69], who found that Taylor's approximation breaks down for $I>0.5$. Risius [53] requires that $I<0.25$, which is based on results of Favre et al. [17]. One way to ensure that $I$ is sufficiently small, is to cut the time series into short segments. The length of the segments limits the size of the largest scale that can contribute to $u^{\prime}$, so shorter segments yield a smaller $u^{\prime}$. Furthermore, after segmenting the time series, segments with a too large I can be rejected. The latter was done in the work by Risius et al. [52]; they used segments of between 1 and 2 minutes long, selected such that $I<0.25$.

In this chapter a sonic anemometer is used to measure the flow close to the particle tracking experiment. Risius' method for computing turbulence statistics [52] is implemented, and is used to compute the dissipation rate $\epsilon$ and the Taylor Reynolds number $\operatorname{Re}_{\lambda}$. The method is applied to data collected between 1-8-2019 and 1-10-2019, and as an example the evolution of the dissipation rate and Taylor Reynolds on 25-9-2019 is plotted.

It is speculated here, that by selecting segments with a limited $I$, the flow conditions for which turbulence statistics are computed are biased, and therefore the resulting statistics might be biased. To see how strong this effect is, the results of Risius' method are superficially compared to results obtained with an alternative method, that does not condition on $I$.

\subsection{Setup}

The instantaneous flow is measured using a $3 \mathrm{D}$ sonic anemometer. It is mounted on a post approximately $1.5 \mathrm{~m}$ to the South of the LPT measurement volume. This way it is located close to the LPT measurement volume, yet doesn't disturb the flow through the volume. The sonic used here is an “Ultrasonic Anemometer 3D" (part no. 4.3830.20.340, Thies Clima, Göttingen), shown in figure 6.1

The sonic's three acoustic paths are located symmetrically around a central post, and are separated by $20 \mathrm{~cm}$. Due to this arrangement, the sonic measures the components of the instantaneous velocity in different locations, and as a 


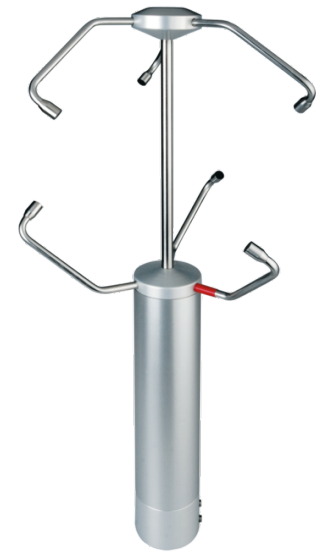

(a) North view

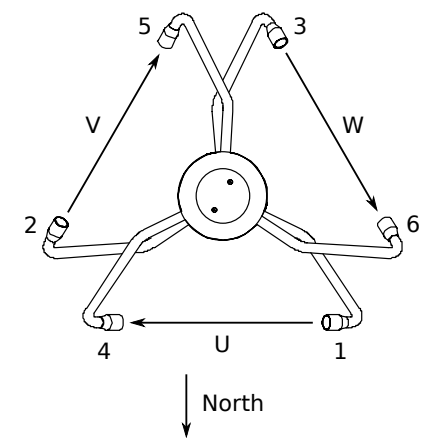

(b) Top view

Figure 6.1: Thies Clima 3D sonic anemometer, part number 4.3830.20.340. The sonic consists of three pairs of ultrasonic transducers, that measure wind speed along the $U, V$, and $W$ directions. They are located around a central support structure. The acoustic paths are $20 \mathrm{~cm}$ away from each other. The transducers pulse in the order indicated by the numbers. In the photo, transducer no. 1 is marked with red tape (grey in print). Photo and drawing from Thies Clima. 
result misrepresents flow features that are of the size of the sonic. For example, if a vortex of $\sim 30 \mathrm{~cm}$ diameter would be centred on the sonic, then instead of measuring zero velocity, which would be the correct average, the sonic would report flow either up or down, depending on the vortex's direction of rotation.

Even though the sonic outputs velocities along the $x, y$, and $z$ directions, these can be rotated back into the sonic's native $U V W$ coordinate system. By doing so, one essentially trades a single 3D measurement for three $1 \mathrm{D}$ measurements. The latter, however, are more localised in space, and might be more suitable for computing e.g. structure functions. Many turbulence statistics are computed either along the mean flow direction, or perpendicular to it, by applying a suitable transformation. But since the coordinate system $U V W$ is fixed to the sonic, the only way to change this coordinate system is to physically reorient the sonic. Since the wind at the Schneefernerhaus mostly comes from either East or West, the sonic was mounted with its $\mathrm{U}$ path perpendicular to this direction, i.e. with its North indicator pointing West. This allows for computing transversal structure functions using only the sonic's $U$ velocity component. The relation between velocities in the $x y z$ and $U V W$ coordinate systems is given by

$$
\left(\begin{array}{l}
u_{x} \\
u_{y} \\
u_{z}
\end{array}\right)=\frac{1}{\sqrt{6}}\left(\begin{array}{ccc}
2 & -1 & -1 \\
0 & -\sqrt{3} & \sqrt{3} \\
\sqrt{2} & \sqrt{2} & \sqrt{2}
\end{array}\right)\left(\begin{array}{l}
u_{U} \\
u_{V} \\
u_{W}
\end{array}\right) .
$$

The rotation matrix was derived by assuming $U, V$, and $W$ are perpendicular to each other and rotationally symmetric around the $z$ axis.

The sonic communicates using a text-based protocol over RS-485. This is used both to configure the sonic and to acquire velocity data. The sonic is configured to output its internal millisecond counter (29 bits, wraps around every 6 days), and $u_{x}, u_{y}$, and $u_{z}$, as often as it can. In practice this means that it measures at $\sim 250 \mathrm{~Hz}$.

The master computer runs a Python code that receives telegrams from the sonic, interpretes these, and saves the counter value and velocity values to a CSV file. The code saves at most one million telegrams to a CSV file; after one million telegrams it creates a new file, the name of which is the current date and time in ISO 8601 format. An excerpt of such a file is shown in listing 6.1

In some cases the sonic fails to output correct values, after which it stutters, and the measurement frequency temporarily reduces to $\sim 30 \mathrm{~Hz}$; a typical case is shown in listing 6.2 In other cases the sonic measures a spike, which could be caused by a drop hitting on of the transducers. A typical example of this is shown in table 6.1. By rotating the results back into the sonic's 
Listing 6.1: Typical output of the sonic. The first column is an internal millisecond counter, that wraps back to zero every $2^{29} \mathrm{~ms}$, or 6 days. The following three columns are the components of the instantaneous velocity in $\mathrm{m} \mathrm{s}^{-1}$.

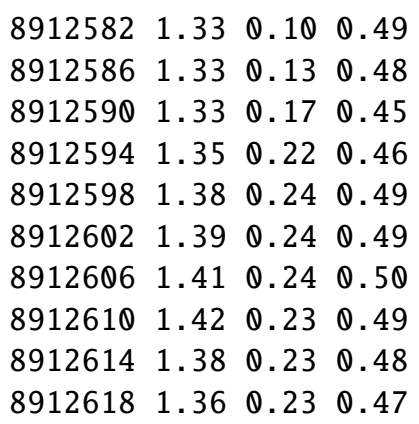

Listing 6.2: Typical occurence of the sonic stuttering. The sonic outputs invalid values, which the data logging code stores in raw form as a comment. After that the sonic needs some time to recover. Note how the counter value doesn't increment by 3 or 4 , as is normal, but by 57 or 58 for the next three lines.

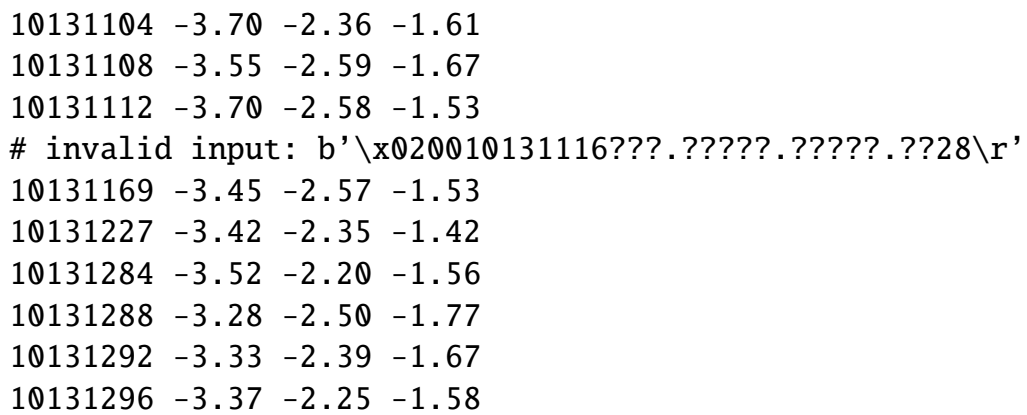


Table 6.1: Typical occurence of a spike, shown in both the $x y z$ coordinate system, and the sonic's native UVW coordinate system. The spike mostly affects just one acoustic path; the U path in this case.

\begin{tabular}{rrrrrrr}
\hline Counter & $u_{x}$ & $u_{y}$ & $u_{z}$ & $u_{U}$ & $u_{V}$ & $u_{W}$ \\
\hline 10943781 & -4.18 & -5.07 & -0.48 & -3.69 & 5.01 & -2.16 \\
10943785 & -4.19 & -4.74 & -0.39 & -3.65 & 4.84 & -1.87 \\
10943789 & -4.28 & -4.56 & -0.27 & -3.65 & 4.82 & -1.63 \\
10943793 & 42.61 & -2.19 & 30.72 & 52.53 & 1.89 & -1.21 \\
10943797 & -4.94 & -4.54 & -0.44 & -4.29 & 4.97 & -1.45 \\
10943802 & -4.99 & -5.03 & -0.75 & -4.51 & 5.16 & -1.95 \\
10943806 & -5.45 & -4.51 & -0.41 & -4.69 & 5.18 & -1.20 \\
\hline
\end{tabular}

native coordinate system UVW, it can be seen that the spike is mostly, but not completely, limited to just one component of the velocity. That it is not completely limited a single component, could mean that the assumption that $U, V$, and $W$ are perpendicalar is incorrect.

Finally it must be mentioned that the sonic's sampling rate isn't constant: in warmer air sound travels faster, hence the sonic measures faster. A temperature increase of $25^{\circ} \mathrm{C}$ causes the sonic to measure $1 \%$ faster.

\subsection{Data analysis}

The data analysis is comprised of a few steps:

1. Make an index of sections of continuous measurements, and unwrap the counter value.

2. Preprocess the data to remove spikes, detect gaps, and ensure a constant sampling rate.

3. Cut the data into segments, and group similar segments together.

4. Compute $R, D_{i i}$, and $\epsilon$ within each group.

5. Create daily graphs from group statistics.

Risius enforced the requirement that $I<0.25$ in step 3. In this work his approach will be followed, as well as an alternative method that does not condition on the turbulent intensity. Note that the only difference between 
Risius' approach and the alternative method, is in step 3. Each of the steps is described in more detail below.

\subsubsection{Indexing}

A period of continuous measuring yields a series of CSV files with steadily increasing counter values. It is assumed that whenever the sonic resets, the Python code is restarted too, such that discontinuities in the counter value never occur within one CSV file; only between CSV files. The questions that should be answered in this section, then, are:

- Which sequences of files correspond to a continuous measurement?

- How do the counter values in each sequence relate to the actual time?

To answer these questions a Python code was written, that reads the CSV files and creates an index into them. Later, the index is used to, given a date and time, determine in which file to look, and what the corresponding counter value is. The index is built as follows:

1. Start with a sorted list of CSV files.

2. Create a sequence, currently without any files, and set this sequence's counts-per-second, or cps, to 1000.

3. Take the first CSV file, and add it to the sequence. Set the sequence's starting time to the CSV file's file name. Set the sequence's starting counter value to that in the file's first line.

4. Take the next CSV file, and compute the time difference $\Delta t$ between this file's starting time, as encoded in the filename, and the sequence's starting time. Predict this file's first counter value with cps $\cdot \Delta t$.

5. Read the counter value of the file's first line. If it is less than the first counter value of the previous file, it must have wrapped, so unwrap it as needed. Compare the unwrapped counter value to the predicted counter value; if the difference is smaller than 5000 counts, append this file to the sequence and continue to the next step. Otherwise, use this file to start a new sequence, and go to step 3.

6. Compute the number of counts $\Delta c$ since the start of the sequence, and update this sequence's cps: $\mathrm{cps}=\Delta c / \Delta t$. Go to step 4 . 
Finally, for each sequence also compute its end time. This is done by reading the sequence's last file's last counter value, unwrapping it, and multiplying it with the sequence's cps.

\subsubsection{Preprocessing}

Once the index is built, the data can be preprocessed. The aim of this step is to remove spikes, detect gaps (as happens when the sonic stutters; see listing 6.2. and resample the data onto an equidistant temporal grid.

The final sampling rate is $10 \mathrm{~Hz}$. This is much lower than the sonic's sampling rate, however due to the size of the sonic, and the distance between the acoustic paths, one can argue that the high sampling rate does not represent the data rate. At a typical mean wind speed of $4 \mathrm{~m} \mathrm{~s}^{-1}$, an eddy of $30 \mathrm{~cm}$, which is the size of the sonic, needs $0.075 \mathrm{~s}$ to pass. Hence, the Nyquist frequency shouldn't be any higher than $\sim 13 \mathrm{~Hz}$. Risius obtained good results with $10 \mathrm{~Hz}$, so that sampling frequency is used here too.

The preprocessing proceeds as follows:

1. Spikes are detected and replaced with NaNs. To do this, each sample is compared to the median of itself, its previous three neighbours, and its next three neighbours. If the current sample deviates by more than $1 \mathrm{~m} \mathrm{~s}^{-1}$, it is considered to be an spike. This threshold was obtained from a histogram.

Note that this approach is technically not quite right: if the current sample borders on a gap, the median computation will span the gap, and hence might be skewed. In such a case up to $20 \mathrm{~ms}$ of data would be lost, which is not worth the effort of saving.

2. Gaps are detected and marked. A gap is detected whenever two subsequent samples are more than $6 \mathrm{~ms}$ apart. Each gap is then filled with a series of NaNs at the average sampling rate.

3. The data is interpolated onto a $1 \mathrm{kHz}$ grid. Because the data is upsampled by a factor 4 , very unsophisticated interpolation schemes suffice: here linear interpolation is used.

4. A low-pass filter is applied to the data, to prevent aliasing in the next step. The low-pass filter used here is a 201-point Hann window. It falls off strongly at $5 \mathrm{~Hz}$. Since applying a window function is essentially the same as computing a weighted average, NaNs can simply be left out. 
At least $80 \%$ of the surface under the Hann window is required to be present, otherwise a $\mathrm{NaN}$ is output.

5. The data is downsampled, by simply taking every 100 th point and throwing the rest out. This yields a sampling rate of $10 \mathrm{~Hz}$.

\subsubsection{Segmentation}

In order to deal with the non-stationarity of the flow, the data is cut into segments, and similar segments are grouped together, or "binned". For each segment, Risius required that the turbulent intensity $I<0.25$. This method might favour certain flow conditions over others and bias the resulting turbulence statistics. To see if this is the case, Risius' method is compared to an alternative method that does not condition on $I$.

Segmentation is done as follows:

- Risius' method:

1. Start with a window that spans the first minute of data.

2. Compute the turbulence intensity $I$ within the window. If $I<0.25$, repeatedly extend the window by a data point until $I$ becomes too large, or the window is 2 minutes long. Use that window to cut out a segment. Define a new 1-minute-long window, starting at the end of the current window, and repeat this step.

3. If, initially, I was too large already, then move the start and end of the window by one data point. Repeat this until $I<0.25$. At that point, go to step 2 .

- Fixed 2-minute segments: all segments are 2 minutes long and overlap by $75 \%$. One hour of data yields 120 segments.

Each segment is required to have no more than $10 \%$ NaNs, otherwise it is discarded.

The code is currently dealing with individual segments, and at some point per-segment statistics need to be computed, so that might as well be done now. Each segment is processed as follows:

1. The mean velocity $\boldsymbol{U}^{(1)}$ is computed and subtracted, so that the segment has zero mean velocity. The superscript indicates that this value pertains to a single segment. 

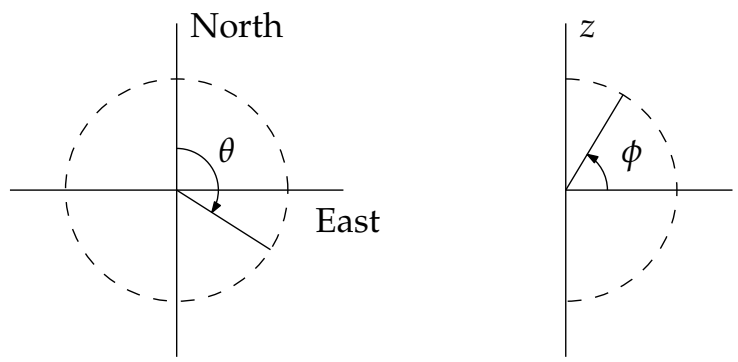

Figure 6.2: Definition of $\theta$ and $\phi$ used for the mean wind direction. Note that $\theta$ and $\phi$ indicate where the wind is coming from, as is common in meteorology.

2. Based on the mean velocity vector a new coordinate system is computed, such that $\hat{\boldsymbol{e}}_{1}$ points along the mean velocity $\boldsymbol{U}^{(1)}, \hat{\boldsymbol{e}}_{2}$ is perpendicular to $\hat{\boldsymbol{e}}_{1}$ and $\hat{\boldsymbol{e}}_{z}$, and $\hat{\boldsymbol{e}}_{3}$ is normal to both previous vectors. The segment is rotated into this new coordinate system.

3. The correlation functions $R_{i i}^{(1)}$ are computed. For the moment these are treated as functions of time delay $\tau$, and not as functions of spatial increment $r$. They are computed for $\tau<30 \mathrm{~s}$, so that only half to a quarter of the storage is needed.

Per segment the following quantities are saved: $\boldsymbol{U}^{(1)}, R_{i i}^{(1)}$, and the segments's time. The rms velocity is not stored, because it can be obtained from $R_{i i}^{(1)}(0)$.

\subsubsection{Binning}

For each segment the mean velocity vector and turbulence intensity are computed. The mean velocity vector is decomposed into magnitude $U^{(1)}$ and angles $\theta$ and $\phi$ as defined in figure 6.2. Each segment is then assigned to a bin in a $4 \mathrm{D} U^{(1)}-\theta-\phi-I$ histogram. For Risius' method so few segments are available, that it was decided to do no binning for $\phi$. This should help the averages in each bin converge better. The binning used for each method is described in table 6.2

\subsubsection{Computing turbulence statistics}

Within each bin the turbulence statistics are computed as follows: 
Table 6.2: All segments with similar turbulence statistics are grouped by assigning them to bins of a $4 D$ histogram on $U^{(1)}, \theta, \phi$, and I. The minumum and maximum values, as well as the bin sizes, are shown here. For Risius' method no binning for $\phi$ is done, which essentially enlarges the bins, which helps statistical convergence.

\begin{tabular}{cccc}
\hline Quantity & Range & Bin size & Unit \\
\hline \multicolumn{5}{c}{ Risius segments } \\
$U^{(1)}$ & $0-10$ & 0.1 & $\mathrm{~m} \mathrm{~s}^{-1}$ \\
$\theta$ & $0-360$ & 30 & $\circ$ \\
$\phi$ & \multicolumn{3}{c}{ (no binning) } \\
$I$ & $0-0.25$ & 0.025 & - \\
\hline \multicolumn{5}{c}{ Fixed-length segments } \\
$U^{(1)}$ & $0-10$ & 0.1 & $\mathrm{~m} \mathrm{~s}^{-1}$ \\
$\theta$ & $0-360$ & 30 & $\circ$ \\
$\phi$ & $0-90$ & 20 & $\circ$ \\
$I$ & $0-2$ & 0.1 & - \\
\hline
\end{tabular}

1. The following averages are computed: the mean velocity $U=\left\langle U^{(1)}\right\rangle$ and the correlation function $R_{i i}(\tau)=\left\langle R_{i i}^{(1)}(\tau)\right\rangle$, where the averages run over all segments in this bin.

2. Taylor's frozen-turbulence approximation is applied by setting $r=\tau \cdot U$.

3. The 2nd-order structure functions are computed from the average correlations functions as follows: $D_{i i}(r)=2\left[R_{i i}(0)-R_{i i}(r)\right]$.

4. For each $D_{i i}$, with $i=1,2,3$, the maximum of $D_{i i} / r^{2 / 3}$ is found and used to obtain three dissipation rates $\epsilon_{i}$.

5. The dissipation rate $\epsilon$ is computed as the average of $\epsilon_{1}, \epsilon_{2}$, and $\epsilon_{3}$.

6. The Reynolds number is computed as $\operatorname{Re}_{L}=\frac{k^{2}}{\epsilon v}$, with $k=\frac{1}{2}\langle\boldsymbol{u} \cdot \boldsymbol{u}\rangle$ the turbulent kinetic energy. The Taylor Reynolds number is computed as $\operatorname{Re}_{\lambda}=\sqrt{20 / 3 \cdot \operatorname{Re}_{L}}$.

7. The integral times are computed using two different methods:

- by fitting $\exp \left(-\tau / T_{i i}\right)$ to $R_{i i}(\tau) / R_{i i}(0)$; and 
- by integrating $R_{i i}(\tau) / R_{i i}(0)$ from 0 to the first zero-crossing, or, if there is no zero-crossing, to the largest $\tau$ for which $R$ has been computed.

These methods give $T_{i i \text {,fit }}$ and $T_{i i \text {,int }}$.

8. The integral lengths $L_{i i}$ are computed as $T_{i i \text {,int }} \cdot U$. The $T_{i i \text {,int }}$ are used, because they capture non-exponential behaviour of the correlation functions better than the $T_{i i \text {,fit }}$ do.

9. The spectra $E_{i i}(\kappa)$ are computed as the type-I discrete cosine transform of $R_{i i}(r) / R_{i i}(0)$.

\subsubsection{Creating daily graphs}

By assuming that the statistics computed within each bin, apply not just to the bin, but also to each of the segments within it, the turbulence statistics can be graphed as a function of time. An example is shown in the next section.

\subsection{Results}

\subsubsection{Segmentation and binning}

Data was collected between 1 August 2019 and 1 October 2019, and was processed as described in the previous section. Risius' method yielded a total of 18565 segments, which averages to one segment every 4.7 minutes. This means that only $\frac{1}{5}$ to $\frac{2}{5}$ of the data satisfies $I<0.25$; the rest is not used in this analysis. Risius does not mention how much of his data he had to reject due to this requirement, so it is not possible to make a comparison.

The fixed-length methods yielded $\sim 170000$ segments.

\subsubsection{Per-bin statistics}

An example of the correlation functions, compensated second-order structure functions, and energy spectra is shown in figure 6.3. The quantities shown are computed for the most populated bin in Risius' method; in that bin $U$ is between $3.3 \mathrm{~m} \mathrm{~s}^{-1}$ to $3.4 \mathrm{~m} \mathrm{~s}^{-1}, \theta$ is between $255^{\circ}$ to $285^{\circ}, I$ is between 0.225 to 0.250 , and it contains 115 segments. The dissipation rate is estimated to be $0.034 \mathrm{~m}^{2} \mathrm{~s}^{-3}$. 

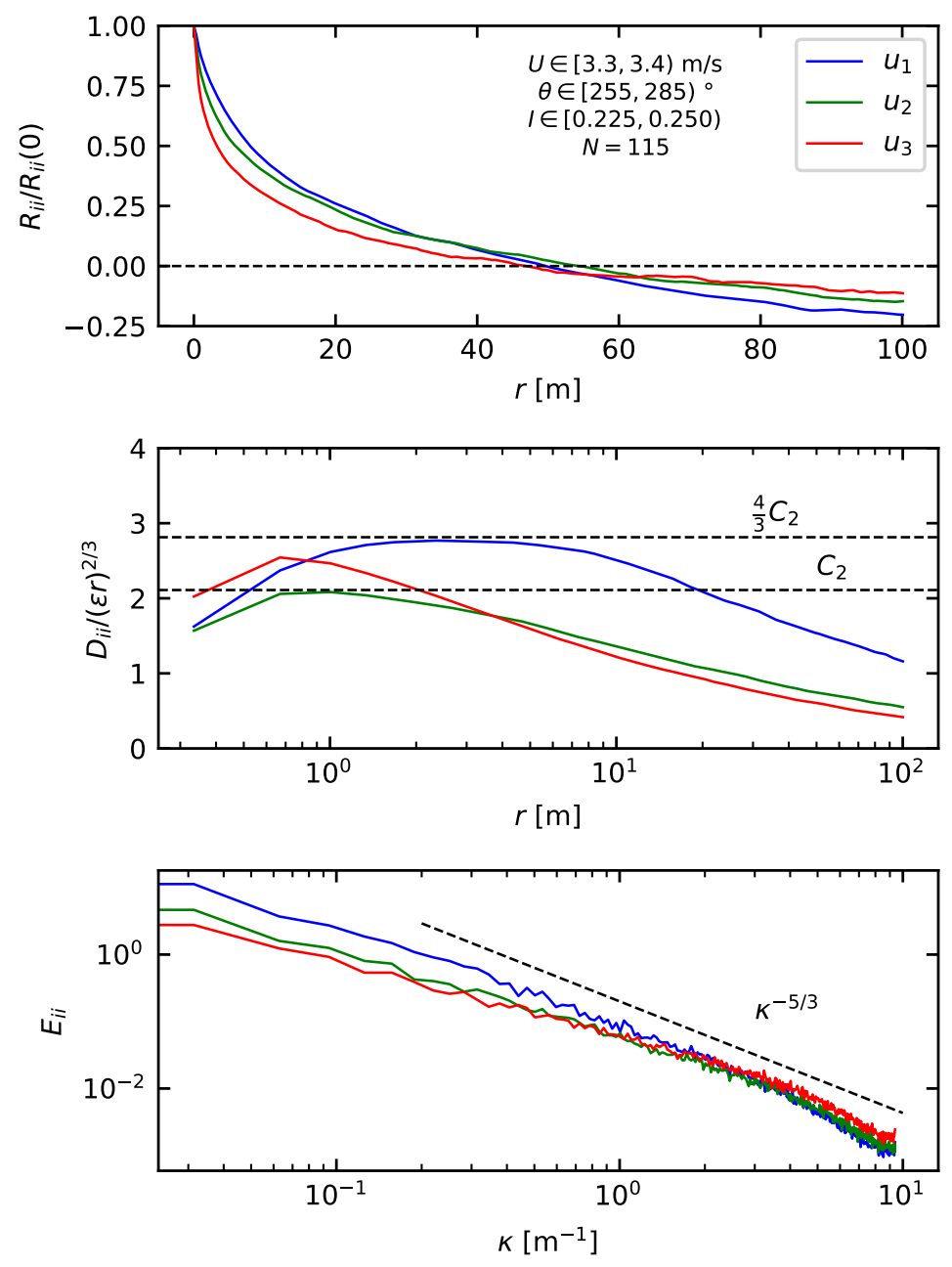

Figure 6.3: Correlation functions (top), compensated second-order structure functions (middle), and energy spectra (top) computed within the most populated bin. This bin contains 115 segments in total; its edges are described in the top graph. The dissipation rate is estimated to be $0.034 \mathrm{~m}^{2} \mathrm{~s}^{-3} . u_{1}$ is in the direction of the mean wind; $u_{2}$ is in the horizontal plane and perpendicular to $u_{1} ; u_{3}$ is perpendicular to both. 
The integral lengths in this bin are $L_{11}=13 \mathrm{~m}, L_{22}=12 \mathrm{~m}$, and $L_{33}=9 \mathrm{~m}$. In isoptropic turbulence $\frac{1}{2} L_{11}=L_{22}=L_{33}$, which is not quite the case here and suggests that the flow is not isotropic.

The compensated structure functions should show plateaus that correspond to the inertial range. Here, $D_{11}$ shows a plateau, albeit only half a decade wide. In isotropic turbulence the plateau of $D_{11}$ should lie at $\frac{3}{4}$ of the height of the plateaus of $D_{22}$ and $D_{33}$, however $D_{22}$ and $D_{33}$ do not show clear plateaus, and their peaks lie below the plateau of $D_{11}$. This could again be attributed to anisotropy.

The longitudinal energy spectrum $E_{11}$ tends to $\kappa^{-5 / 3}$ over approximately one-and-a-half decade. The transversal energy spectra $E_{22}$ and $E_{33}$ seem to have a slightly less negative exponent.

\subsubsection{Daily graphs}

The evolution of the dissipation rate on 25-9-2019 is shown in the top two graphs of figure 6.4. The top graph shows the values obtained using Risius' method, while the middle graph shows values obtained with fixed-length segments. The shaded area indicates when the particle tracking experiment was running. Some trends can be seen, which may be related to changes in the weather. Other than that, the dissipation rate fluctuates strongly.

In order to compute Stokes numbers in chapter 7 a dissipation rate must be determined for each individual particle tracking experiment. This can be done in various ways:

- The simplest way is to take the dissipation rate from the segment closest in time to the LPT experiment of interest. However, Risius' method does not guarantee that segments are produced at regular intervals, so the time difference between an LPT experiment and the closest segment can be arbitrarily large. As a result, dissipation rates determined this way may not be representative of the flow at the time of the LPT experiment.

- Another method would be to interpolate the dissipation rate between segments.

- The method used here, is to fit a piecewise linear function to the dissipation rate. To handle the irregular intervals between the segments in Risius' method, each segment $i$ gets assigned a weight $w_{i}$ equal to half time difference between its two neighbouring segments, i.e. $w_{i}=\left(t_{i+1}-t_{i-1}\right) / 2$. 

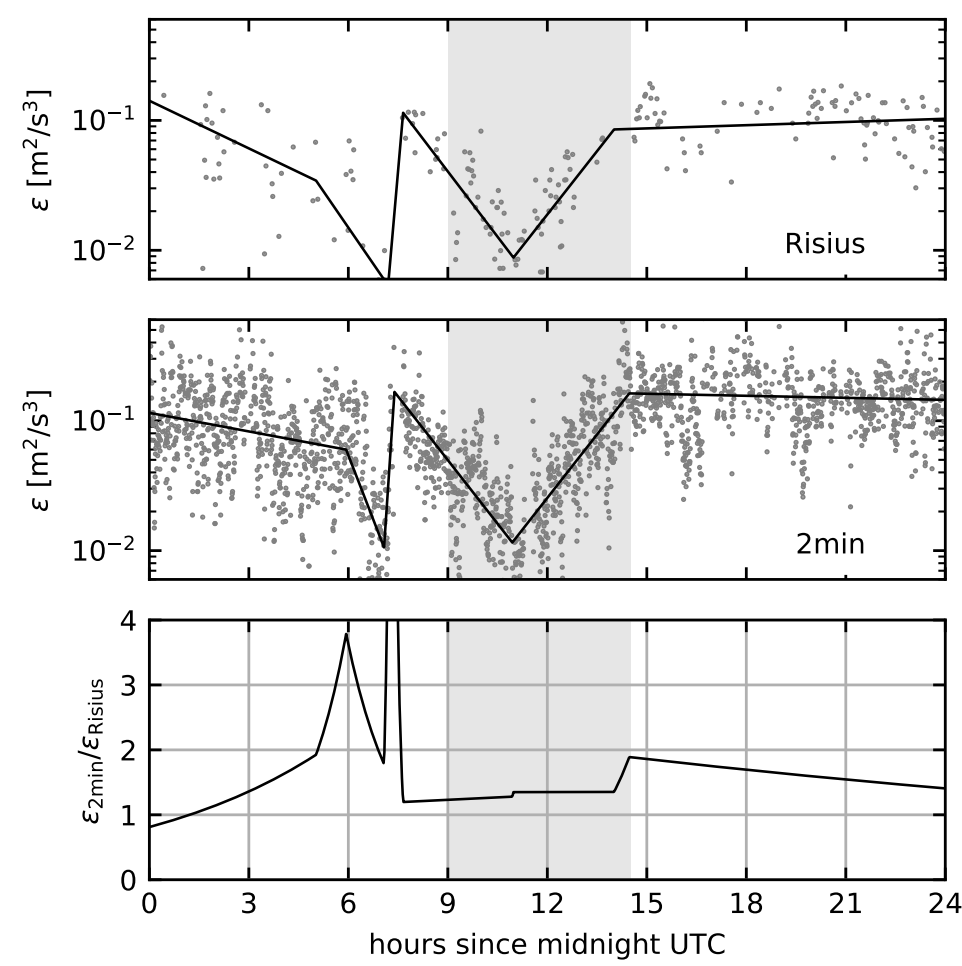

Figure 6.4: Evolution of the dissipation rate on 25-9-2019, computed with Risius' method (top) and fixed-length segments (middle), as well as the ratio between these (bottom). The solid line in the top and middle graph is a piecewise linear fit to $\ln (\epsilon)$. The bottom graph is the ratio between the fits. The shaded area indicates when the particle tracking experiment was running. 


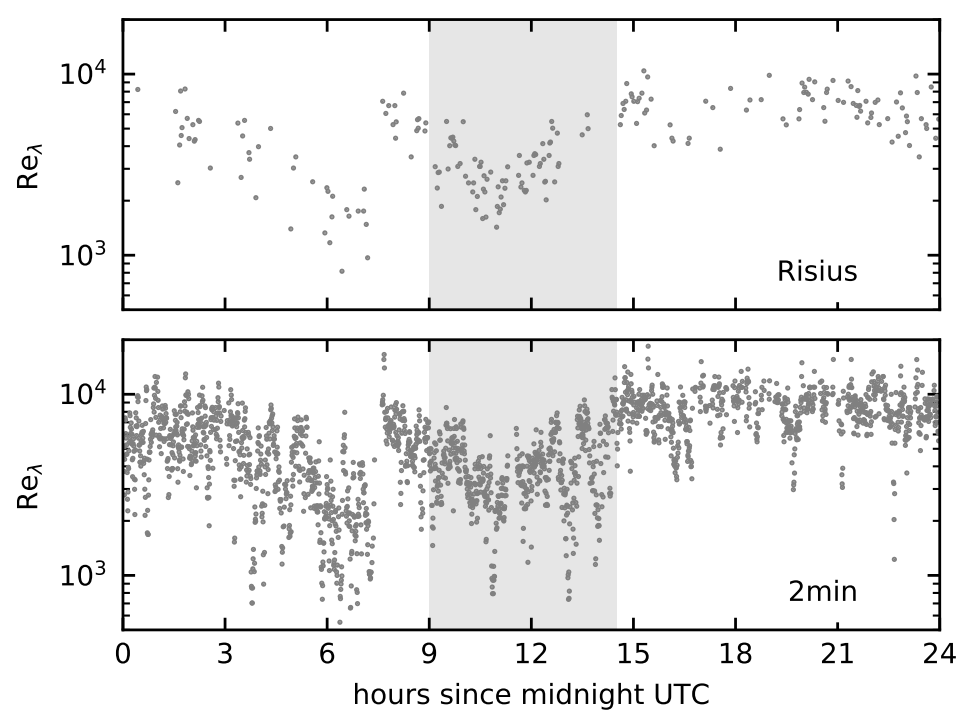

Figure 6.5: Evolution of the Taylor Reynolds number on 25-9-2019, computed with Risius' method (top) and fixed-length segments (bottom). The shaded area indicates when the particle tracking experiment was running.

Since the dissipation rate is expected to be log-normally distributed, the fits are fits to $\ln (\epsilon)$. The resulting fits for 29-5-2019 are shown in figure 6.4

The fits allow for convenient comparison between the dissipation rates obtained with both methods. The bottom graph of figure 6.4 shows the ratio $\epsilon_{2 \mathrm{~min}, \mathrm{fit}} / \epsilon_{\text {Risius,fit, }}$ and as can be seen the 2-minute method yields larger dissipation rates. This is not surprising: not conditioning on $I$ means that larger rms velocities $u^{\prime}$ are admissible, and since $\epsilon \propto u^{\prime 3} / L$, larger dissipation rates should be expected too.

The evolution of the Taylor Reynolds number on 25-9-2019 is shown in figure 6.5. The exact value of the Taylor Reynolds number is not as important, so fits to these data have not been made. The Taylor Reynolds number during the particle tracking experiments on 25-9-2019 can be estimated to be between $2 \times 10^{3}$ and $6 \times 10^{3}$. 


\subsection{Conclusion}

A sonic anemometer is used to measure the flow at the Schneefernerhaus, close to the particle tracking experiment. In order to deal with non-stationarity and to limit the turbulent intensity, the data is cut into short segments using two different methods: one introduced by Risius et al. [52], that requires $I<0.25$, and one that doesn't condition on $I$. Similar segments are then grouped together (binned), and the dissipation rate and Taylor Reynolds number for each bin are computed and mapped back onto the contributing segments.

The resulting dissipation rates may show trends that correspond to changes in the weather, but otherwise fluctuate strongly. These fluctuations are smoothed out using piecewise linear fits, that will be used in chapter 7 to compute an effective dissipation rate for each particle tracking experiment.

Initially only Risius' method was to be used to measure the dissipation rate and the Taylor Reynolds number. But, as is discussed in the next chapter, in section 7.1.3. the dissipation rates measured with Risius' method are so low, that the droplet acceleration statistics siginificantly deviate from literature values. Furthermore, because $\epsilon \propto u^{\prime 3} / L=(I U)^{3} / L$, it can be argued that discarding data with large turbulent intensities biases the results towards lower dissipation rates. Therefore, it was decided to include statistics computed from segments that are not conditioned on turbulent intensity. It must be noted, though, that these statistics are plagued by high turbulent intensities, and should be considered preliminary.

The effect of a high turbulent intensity on the applicability of Taylor's approximation has been studied extensively; see e.g. [18, 30, 37, 39, 70]. Both Lumley [39] and Hill [30] give corrections, but these corrections go in the wrong direction: given the true spatial dependency of some quantity $Q(r, 0)$, they predict the temporal dependency $Q(0, \tau)$ as it would be measured. A literature study must be done to see if the proposed corrections have been applied to measurements in atmospheric flows already. If not, it would be interesting to do so, or to understand why it cannot be done.

Risius was mostly interested in the distributions of various turbulence statistics, and for that reason it was natural for him to segment and bin the data, and to compute the statistics per bin. While this method can be used to obtain instantaneous values for the turbulence statistics, it is perhaps less effort and/or more reliable to compute instantaneous turbulence parameters directly, as is done in e.g. [61.

Instead of using a single sonic anemometer, the turbulence statistics could also be measured with an array of sonic anemometers. This way the instantenous velocity field can be measured directly, without having to invoke Taylor's 
approximation. Using e.g. 4 sonic anemometers, the transverse second-order structure function can be measured for 6 different separations $r$. The idea behind this is not new, and has been applied to wind tunnels using hot-wire probes before [11, 27]. 


\section{Chapter 7}

\section{Droplet dynamics}

In this chapter the elements of all three previous chapter come together: various analysis will be performed on the droplet trajectories, taking their sizes into account through the Stokes number, and taking the turbulence statistics into account, in particular the dissipation rate. The quantities studied here are the droplet accelerations (section 7.1) and the radial distribution function (section 7.2). Even though measured data for the radial relative velocities are available, due to time constraints an analysis of the RRV remains to be done, and the RRV is not presented in this thesis.

The quantities presented here are studied for three days: 25-9-2019, 26-92019, and 28-9-2019. Plots of the dissipation rate and Taylor Reynolds on these days can be found in section E.1 for the reader's convenience approximate values are shown in table 7.1. The dissipation rate in particular is a timedependent quantity; many of the analysis presented here are therefore done per experiment, with the average dissipation rate at the time of the experiment

Table 7.1: Approximate dissipation rates and Taylor Reynolds numbers for the three days studied here. These numbers are approximate, and are provided for the reader's convenience; precise values can be found in the graphs in section E.1

\begin{tabular}{ccc}
\hline Date & $\epsilon\left[\mathrm{m}^{2} \mathrm{~s}^{-3}\right]$ & $\operatorname{Re}_{\lambda}$ \\
\hline $25-9-2019$ & $0.01-0.1$ & 4000 \\
$26-9-2019$ & $0.05-0.1$ & 8000 \\
$28-9-2019$ & 0.04 & 3000 \\
\hline
\end{tabular}


obtained from piecewise linear fits (chapter 6).

\subsection{Accelerations}

\subsubsection{Theory}

Tracer particles, i.e. particles with $\mathrm{St}=0$, follow the flow exactly. As a result, the distribution of their accelerations is equal to that of the fluid,

$$
a_{\mathrm{rms}}^{2}=\frac{1}{3}\langle\boldsymbol{a} \cdot \boldsymbol{a}\rangle=\left\langle\left(\frac{\mathrm{D} u}{\mathrm{D} t}\right)^{2}\right\rangle,
$$

with $\boldsymbol{a}$ the particle accelerations and $\left\langle(\mathrm{D} u / \mathrm{D} t)^{2}\right\rangle$ the single-component rms acceleration of fluid parcels. As the particles get heavier, i.e. as their Stokes number increases, they will preferentially probe regions of the flow with low vorticity. These regions are associated with low accelerations, which is reflected in the particle accelerations. If they get even heavier, their response time $\tau_{p}$ becomes so large, that they essentially can't keep up with the fluid accelerations. Bec et al. [4] relate the particle rms accelerations to that of tracers as follows:

$$
a_{\mathrm{rms}}^{2}(\mathrm{St})=a_{\mathrm{rms}}^{2}(\mathrm{St}=0)+C \exp \left[-(D / \mathrm{St})^{\delta}\right],
$$

with $C$ and $D$ undetermined parameters. The exponent $\delta=2 / 3 \beta$, with $\beta=0.41$ given by Voth et al. [66]. Some expressions for the single-component rms accelerations of tracers can be found in literature:

$$
\begin{aligned}
& a_{0}^{2}=\frac{1}{3}\left(2.5 \operatorname{Re}_{\lambda}^{0.25}+0.08 \operatorname{Re}_{\lambda}^{0.11}\right) \quad \text { Hill [29, eq. (4.2)] } \\
& a_{0}^{2}=\frac{11+7 \operatorname{Re}_{\lambda}}{205+\operatorname{Re}_{\lambda}} \quad \text { Ayala et al. [2, eq. (26)] }
\end{aligned}
$$

Here $a_{0}^{2}=\left\langle(\mathrm{D} u / \mathrm{D} t)^{2}\right\rangle / a_{\eta}^{2}$, with $a_{\eta}=\eta / \tau_{\eta}^{2}=\left(\epsilon^{3} / v\right)^{1 / 4}$ the Kolmogorov acceleration. For $\operatorname{Re}_{\lambda}=3000$, which is the mode of the Taylor Reynolds number at the Schneefernerhaus, these yield $a_{0}=2.5$ and $a_{0}=2.6$, respectively. For comparison, Bec et al. [4] measured $a_{0}^{2}=3.09\left(a_{0}=1.76\right)$ at $\operatorname{Re}_{\lambda}=185$ in their numerical simulation.

In this section equation (7.2) is fitted to the measured rms droplet acceleration. First, the equation is non-dimensionalised as follows:

$$
\frac{a_{\mathrm{rms}}^{2}(\mathrm{St})}{a_{\eta}^{2}}=a_{0}^{2}\left\{1-C \exp \left[-(D / \mathrm{St})^{\delta}\right]\right\},
$$


with $a_{0}$ the non-dimensional single-component rms acceleration of tracers. Furthermore the sign of the exponential term is flipped; this is a purely cosmetical change that helps to emphasise that the rms acceleration decreases as a function of increasing Stokes number. The fit parameters are $a_{0}, C$, and $D$.

\subsubsection{Method}

The following steps are taken:

1. Per experiment: bin droplets by size. The bin edges range from $4 \mu \mathrm{m}$ to $52 \mu \mathrm{m}$ in steps of $4 \mu \mathrm{m}$. Bins with less than $10^{4}$ droplets in it are discarded. Compute the dissipation rate at the time of the experiment using the piecewise linear fits from chapter 6

2. Per experiment, per size bin: compute the Stokes number from the bin centre. Compute $\tilde{a}(\mathrm{St})=a_{\mathrm{rms}} / a_{\eta}$, with $a_{\mathrm{rms}}=\left(\frac{1}{3}\langle\boldsymbol{a} \cdot \boldsymbol{a}\rangle\right)^{1 / 2}$. In the next section it is shown that the measured accelerations are isotropic, so that the component average can be computed.

3. Per experiment: fit equation (7.5) to $\tilde{a}(\mathrm{St})$. This yields a per-experiment value for $a_{0}$.

4. Fit equation (7.5) to all of $\tilde{a}(\mathrm{St}) / a_{0}$, where $a_{0}$ are the per-experiment values obtained in the previous step. This gives $C$ and $D$.

Ideally the fits in the last two steps would be done only once, to all experiments simultaneously. But, as will be shown in the next section, $a_{0}$ various strongly between experiments, so the fit must be computed twice: once per experiment to obtain $a_{0}$, and once for all experiments to obtain $C$ and $D$.

\subsubsection{Results}

The per-component rms accelerations on a single day are shown in figure 7.1 . plots for days can be found in section E.2. The accelerations are averaged over batches of 30 experiments; each colour corresponds to a batch. As can be seen, the $x, y$, and $z$ components are almost equal. This shows that the small scales are isotropic, and that the components can be averaged over.

Furthermore, all of the curves of 25-9-2019 have $a_{\mathrm{rms}} / a_{\eta}$ between 6 and 20 at the lowest Stokes numbers. However, as was discussed previously these curves should go to $a_{0} \sim 2.5$ as the Stokes number decreases. Say, for the sake of argument, that on average $a_{\mathrm{rms}} / a_{\eta}=12$ for the lowest Stokes numbers, 


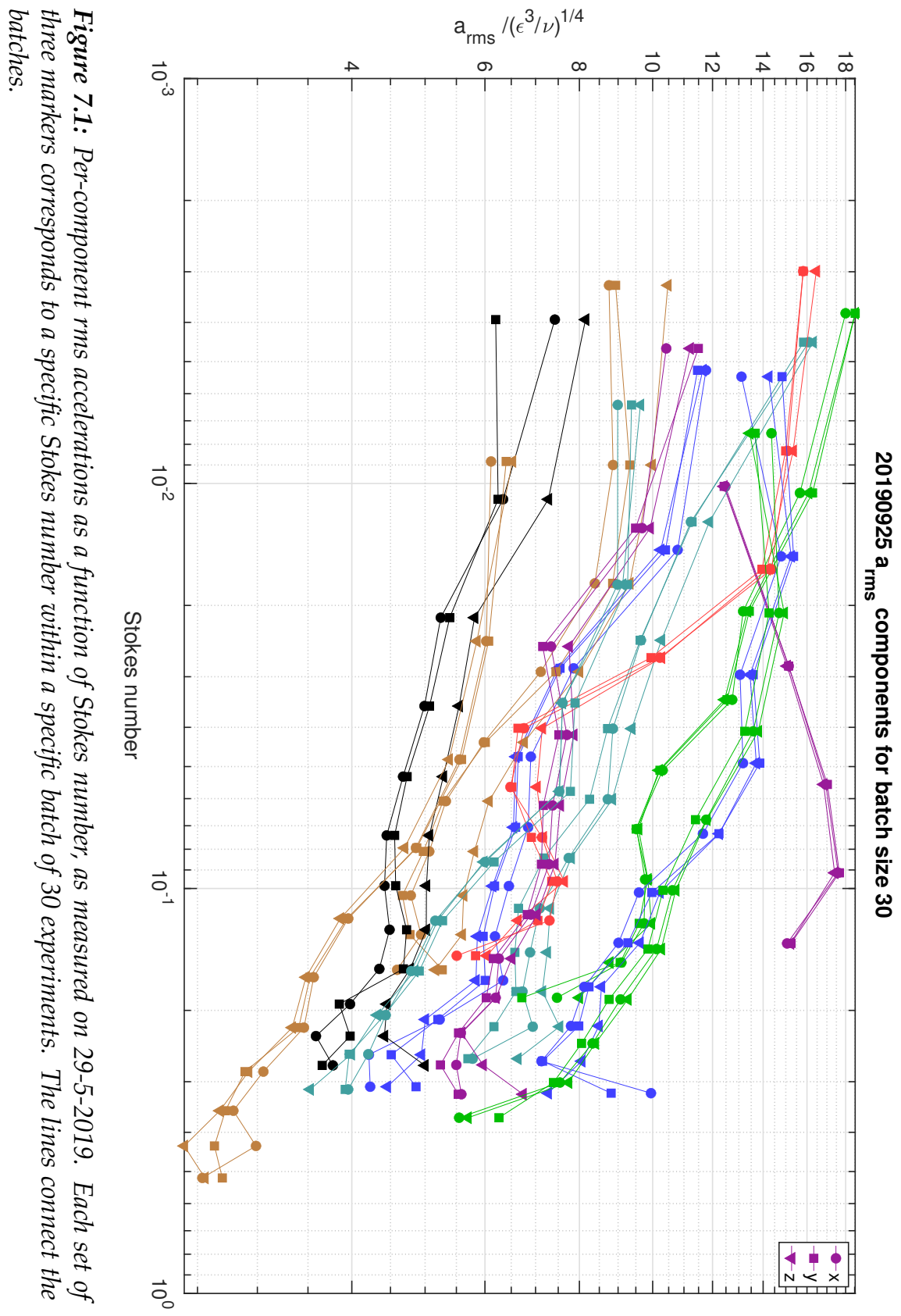




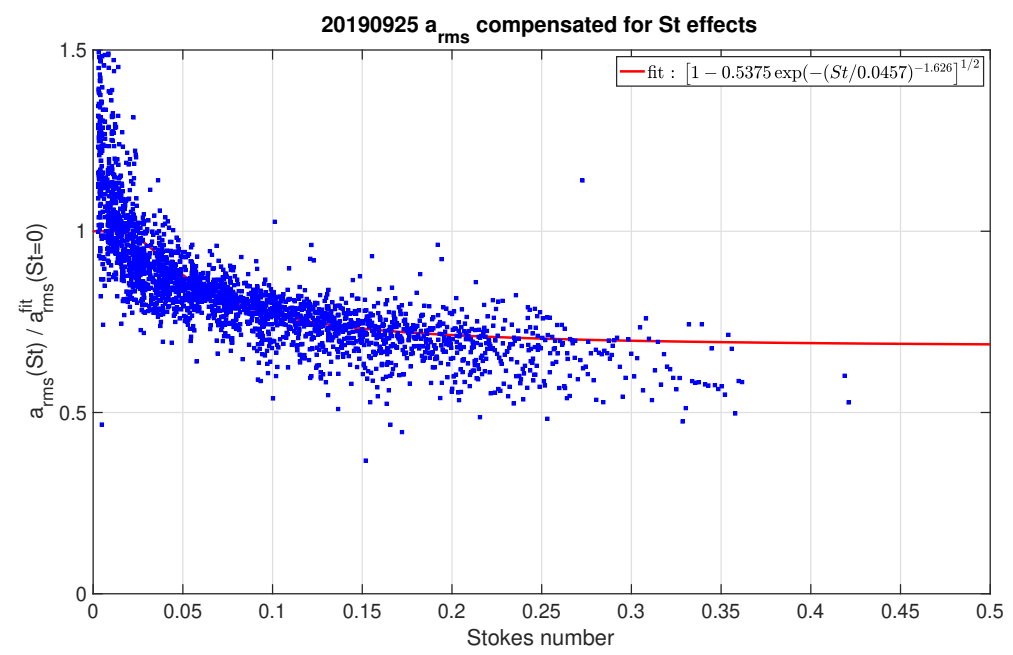

Figure 7.2: Single-component rms accelerations as a function of Stokes number, as measured on 25-9-2019. Each marker corresponds to a specific Stokes number in a specific experiment. The line is fit of equation (7.5) to the data, where $a_{0}$ is allowed to on a different value for each experiment.

then, to get this value down to 2.5 , the dissipation rate must be increased by a factor of $(12 / 2.5)^{4 / 3} \approx 8$.

Finally, these curves show an extremely large spread. Each data point in these plots corresponds to 30 experiments, totalling $100 \mathrm{~s}$ of experimental data. This might be too short to get sufficient statistical convergence, but more study is needed to confirm this.

Figure 7.2 shows a fit of equation (7.5) to the single-component rms accelerations, as computed in the last step described in the previous section. Fits for all days can be found in section E.3. The fit parameters for each day are shown in table 7.2 As can be seen, the model equation fits the data well, but a comparison with literature values is difficult, on account of the differences between the model equation used here, and the one published by Bec et al. 
Table 7.2: Model parameters of equation (7.5) obtained from fits to data from three different days.

\begin{tabular}{ccc}
\hline Date & $C$ & $D$ \\
\hline $25-9-2019$ & 0.5375 & 0.0457 \\
$26-9-2019$ & 0.4517 & 0.1167 \\
$28-9-2019$ & 0.7304 & 0.0132 \\
\hline
\end{tabular}

\subsection{Radial distribution function}

\subsubsection{Theory}

As mentioned in section 2.3.1 most experiments cannot resolve the radial distribution function at droplet-touching distance $r=R$, and the experiment presented here is no exception. Instead, a functional form for the RDF is assumed and fitted to experimental data at $r \gg R$. Chun et al. [12] proposed the following form for the bidisperse RDF:

$$
g_{12}(r)=c_{0}\left[\frac{\eta^{2}+r_{c}^{2}}{r^{2}+r_{c}^{2}}\right]^{c_{1} / 2},
$$

with $c_{0}$ an unspecified proportionality constant, $c_{1}$ the clustering exponent, and $r_{c}$ a cross-over length given by

$$
\frac{r_{c}}{\eta} \approx 5.0\left|\mathrm{St}_{1}-\mathrm{St}_{2}\right|
$$

Two regimes can be identified:

$$
\begin{aligned}
& r \ll r_{c} \quad \Rightarrow \quad g_{12} \approx c_{0}\left[1+\frac{\eta^{2}}{r_{c}^{2}}\right]^{c_{1} / 2} ; \\
& r \gg r_{c} \Rightarrow g_{12} \approx c_{0}\left(\eta^{2}+r_{c}^{2}\right)^{c_{1} / 2}\left(\frac{1}{r}\right)^{c_{1}} .
\end{aligned}
$$

The transition between these regimes is at $r=r_{c}$.

It must be noted that the functional form given by Chun et al. [12] does not hold for $r \gg \eta$ or $r \gg r_{c}$ : for such $r$ the RDF should go to 1 , and not to 0 . To remedy this, the functional form proposed by Chun et al. [12] is modified 
to have an additional asymptotic regime for $r \ll \eta$ :

$$
g_{12}(r)=\left[\frac{r^{2}+r_{c}^{2}+A^{2}}{r^{2}+r_{c}^{2}}\right]^{c_{1} / 2} .
$$

Assuming that $r_{c} \ll A$, the following regimes can be identified:

$$
\begin{aligned}
r \ll r_{c} & \Rightarrow \quad g_{12} \approx\left(\frac{A}{r_{c}}\right)^{c_{1}} ; \\
r_{c} \ll r \ll A & \Rightarrow \quad g_{12} \approx\left(\frac{A}{r}\right)^{c_{1}} ; \\
r \gg A & \Rightarrow \quad g_{12} \approx 1 .
\end{aligned}
$$

The transitions between these regimes are at $r=r_{c}$ and $r=A$.

The model presented here differs from the one by Chun et al. [12]: firstly, it contains no proportionality constant $c_{0}$; if it did, it would not asymptotically go to 1 in the last regime. Second, for the models to be equal in the first two regimes, i.e. subequations (a) and (b), either $A^{2}=\eta^{2}$, or $A^{2}=\eta^{2}+r_{c}^{2}$, which cannot be satisfied simultaneously. Below, the model will be fitted to measured RDFs, with $c_{1}, r_{c}$, and $A$ fit parameters, the aim of which is verify the prefactor in equation (Chun 2.80). However, because of the differences between equations (Chun 2.80) and (7.7), one should be cautious when comparing the fits presented here with literature values.

The model above is not complete without an expression for the clustering coefficient $c_{1}$. Chun et al. [12] obtained an expression for $c_{1}$ based on theoretical considerations. Their resulting expression for $c_{1}$ is a function of $\mathrm{St}_{1}, \mathrm{St}_{2}$, and the average fluid rates of strain and rotation as sampled by the particles. The latter quantity is available in numerical simulations, which they performed, but not in the experiments presented here.

Ayala et al. [2] used an empirical expression for $c_{1}$,

$$
c_{1}=\frac{-0.1988 \mathrm{St}^{4}+1.5275 \mathrm{St}^{3}-4.2942 \mathrm{St}^{2}+5.3406 \mathrm{St}}{\operatorname{Fr}_{\mathrm{f}}^{f_{1}\left(\operatorname{Re}_{\mathrm{A}}\right)}},
$$

with $f_{1}$ a function that tends to 0.1886 for large $\operatorname{Re}_{\lambda}$, and $\mathrm{Fr}_{\mathrm{f}}=u_{\eta} / g \tau_{\eta}$ the fluid Froude number. They do not attempt to justify these expressions.

Here, the following expression is used:

$$
c_{1}\left(\mathrm{St}_{1}, \mathrm{St}_{2}\right)=c_{11}\left[\sqrt{\mathrm{St}_{1} \mathrm{St}_{2}}-c_{12}\left|\mathrm{St}_{1}-\mathrm{St}_{2}\right|\right],
$$


with $c_{11}$ and $c_{12}$ model parameters that are to be determined. For $r_{c}$ the following expression is used:

$$
\frac{r_{c}}{\eta} \approx K\left|S t_{1}-S t_{2}\right|,
$$

with $K$ a model parameter that is to be determined.

\subsubsection{Method}

The following steps are taken:

1. Per experiment: bin droplets by size. The bin edges range from $4 \mu \mathrm{m}$ to $52 \mu \mathrm{m}$ in steps of $4 \mu \mathrm{m}$.

2. Per batch of 30 consecutive experiments: determine the average dissipation rate for these experiments from the piecewise linear fits computed in chapter 6. Use this to compute the Stokes numbers for the centres of the droplet size bins.

3. Per experiment, per size bin pair: compute the RDF. This is done for all possible combinations of bin pairs within an experiment. Bins pairs that cannot form at least $10^{6}$ droplet pair, are skipped.

4. Fit equation (7.7) to the RDFs. In this step $c_{1}$ is a per-RDF fit parameter, and $A$ and $K$ are kept at fixed values. This step is repeated for various combinations of $A$ and $K$, and the combination that yields the best fits for all RDFs is kept.

5. Per day: fit equation 7.10 to all $c_{1}$ obtained in the previous step to determine the parameters $c_{11}$ and $c_{12}$.

For a single particle tracking experiment, given two size bins $d_{p 1}$ and $d_{p 2}$, the RDF $g_{12}$ is computed as follows:

1. For each frame: compute the histogram of pair separations for all $N_{1} N_{2}$ droplet pairs that can be formed between the two bins.

If a monodisperse RDF is requested, i.e. if bin no. 1 and bin no. 2 are the same bin, then $N_{1}=N_{2}=N$, and only $N(N-1)$ unique pairs can be formed.

2. Sum all histograms made in the previous step. The resulting histogram is the unnormalised RDF $g_{12}^{(u)}$. 
3. For each of both bins: discard the temporal info (frame numbers). The result is a collection of homogeneously spread droplets, i.e. without clustering. To limit computational cost required in the next step, randomly select 32768 droplets from each collection, and discard the rest.

If a monodisperse RDF is requested, both collections are identical.

4. Compute the homogeneous, unnormalised RDF of the two droplet collections formed in the previous step. The computation is the same as in step 1. The resulting histogram is the homogeneous, unnormalised $\operatorname{RDF} g_{12}^{(h)}$.

5. Compute the RDF $g_{12}(r)=g_{12}^{(u)}(r) / \alpha g_{12}^{(h)}(r)$, with $\alpha$ some scaling factor to account for the limited number of droplets that was used to compute the RDF in the previous step.

Computing the RDF requires one to normalise the number of droplet pairs by the volume of a spherical shell with radius $r$ (see equation (2.29)). In the particle tracking experiment, part of the spherical shell may lie outside of the measurement volume, and hence may not be counted. This makes computing the normalisation factors in equation (2.29) difficult; much more difficult than computing an (unnormalised) RDF of homogeneously spread droplets, as is done steps $3-5$ above.

\subsubsection{Results}

An example of a number of measured RDFs is shown in figure 7.3. For the case shown in the figure, $\mathrm{St}_{1}=0.105$ and $\mathrm{St}_{2}$ varies. The steep drop in the RDFs at $r<0.2 \mathrm{~mm}$ for $\mathrm{St}_{2}=0.04$ and $\mathrm{St}_{2}=0.07$ is the result of the droplets' close proximity and the low brightness of the second drop: the particle tracking experiment cannot reliably resolve both droplets in such pairs.

Also shown in figure 7.3 are fits of the model equation, equation (7.7), to the measured RDFs. The overall best model parameters are $A=4 \mathrm{~mm}$ and $K=5$. The latter is in line with what was found by Chun et al. [12].

Figure 7.4 shows a fit of equation (7.11) to all clustering coefficients $c_{1}$ measured on 25-9-2019. The fit parameters are $c_{11}=1.4$ and $c_{12}=0.3$. Fit parameters for all days are shown in table 7.3 , the corresponding fits can be found in section E.4. 


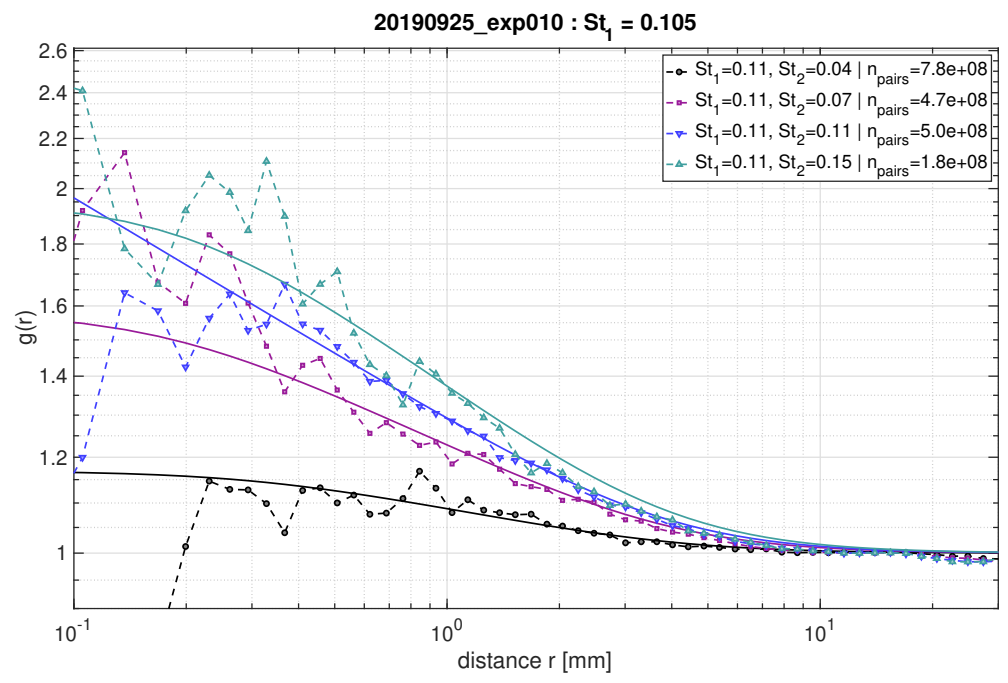

Figure 7.3: Radial distribution functions measured for bidisperse droplets. Here $\mathrm{St}_{1}=0.105$ and $\mathrm{St}_{2}$ varies. The solid lines are fits using the model RDF, equation (7.7).

Table 7.3: Fit parameters of equation (7.10) obtained from fits to data from three different days.

\begin{tabular}{ccc}
\hline Date & $c_{11}$ & $c_{12}$ \\
\hline $25-9-2019$ & 1.40 & 0.3 \\
$26-9-2019$ & 1.04 & 0.3 \\
$28-9-2019$ & 0.96 & 0.3 \\
\hline
\end{tabular}




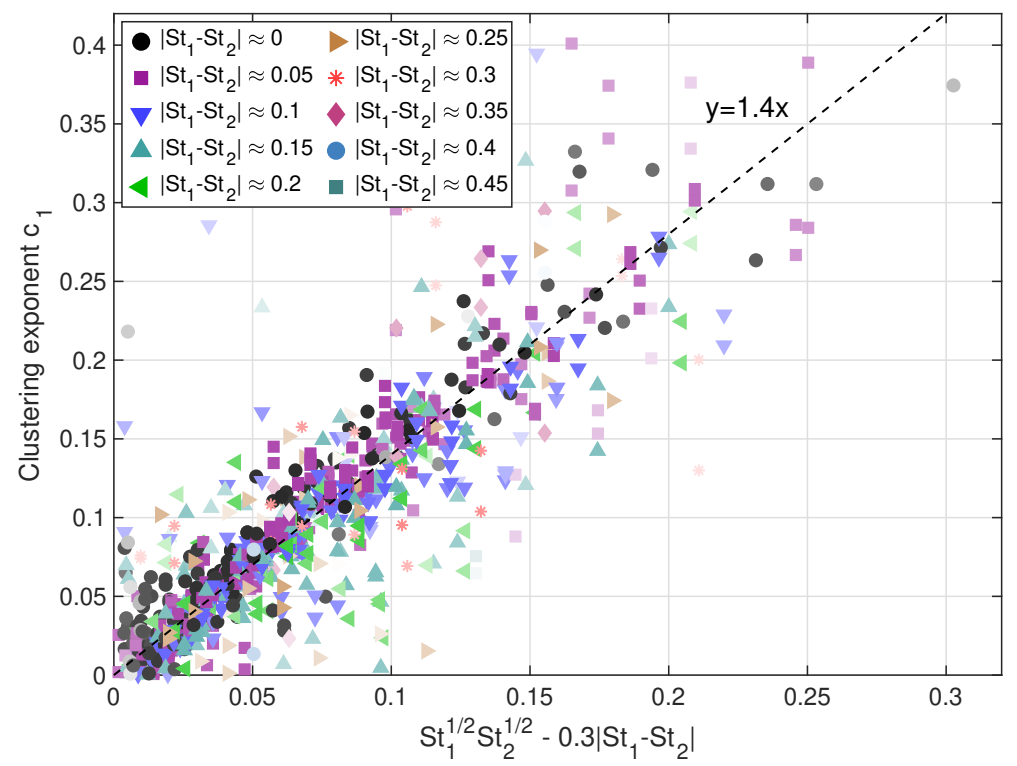

Figure 7.4: Fit of equation (7.10) to all clustering coefficients $c_{1}$ measured on 29-52019. The fit parameters are $c_{11}=1.4$ and $c_{12}=0.3$. 


\subsection{Conclusion}

In this chapter two quantities are computed from measured droplet trajectories: the rms acceleration as a function of the Stokes number, and the radial distribution function. Each of these is fitted with a model from literature, or a variation thereof, and there is general agreement between the data and the models. It must noted though, that all results presented here are preliminary. In particular the dissipation rate needs more scrutiny, because of the much too large rms accelerations.

Many more analyses are left for future work:

- Uncertainties must be computed for all analyses that are performed here.

- There are many more models in literature that could be fitted to the experimental data presented here. Good starting points are Gustavsson and Mehlig [25] and Ayala et al. [2], and references therein.

- There is evidence that clustering occurs mostly in the horizontal plane [21]. This could be verified by conditioning the RDFs on the angle between the separation vector $r$ and the vertical.

- The radial relative velocities. A good starting point are the studies by Saffman and Turner [55], Wang et al. [68] and Ayala et al. [2]. 


\section{Chapter 8}

\section{Conclusion}

In this thesis a unique, in-situ cloud droplet tracking experiment is presented. The experiment is situated on top of the environmental research station Schneefernerhaus, at an altitude of $2650 \mathrm{~m}$ in the German Alps. At this altitude clouds occur naturally. The experiment is augmented with a novel technique to measure cloud droplet sizes, as well as an instrument to measure properties of the turbulent carrier flow, and is used to determine the acceleration statistics and radial distribution functions of cloud droplets.

The particle tracking experiment was described in chapter 4. It is quite conventional in terms of scientific instruments used. What sets the LPT experiment apart from other experiments, is that it is used in-situ. For this reason it is housed in a weather-proof box that is exposed to the environment for several months each year.

The particle videos were analysed with a novel code, called Low-Light LPT $\left(\mathrm{L}^{3} \mathrm{PT}\right)$, that is currently under development by Dr. Moláček. $\mathrm{L}^{3} \mathrm{PT}$ is inspired by the Shake-The-Box algorithm, and is suitable for tracking badly defocused droplets in a densely seeded flow-abilities that are a necessity for the experiment presented here. In addition to particles seen by two or more cameras, $\mathrm{L}^{3} \mathrm{PT}$ also tracks particles seen by only one camera. These cannot be triangulated, but tracking these helps to increase the overall accuracy of the code. Compared to the Shake-The-Box algorithm, $\mathrm{L}^{3} \mathrm{PT}$ expends much more computational cost, but gets higher yield and higher accuracy.

In chapter 6 the turbulence statistics were measured using a method initially developed by Risius et al. [52]. Although the method works and provides plausible dissipation rates, there are reasons to believe that it underestimates the turbulent dissipation rate. Further study should show whether this is the 
case.

Then, in chapter 5, a novel droplet sizing technique was developed, that relates the droplet's intensities as observed by the LPT experiment, to their sizes. Results obtained with the technique agree well with those obtained with the HALOHolo. The technique is not limited to the LPT experiment presented here; it can be applied to any LPT experiment that has spherical particles, and a diffuse light source and/or large camera apertures.

Finally, in chapter 7, all ingredients came together: various analyses were performed on droplet trajectories conditioned on Stokes number. The Stokes numbers, in turn, were computed from the droplet sizes and the turbulent statistics. First, droplet accelerations as a function of Stokes number were compared with literature values, and were found to qualitatively agree, but were far too large in magnitude. This might be caused by an ovestimate of the turbulent dissipation rate, but further investigation is needed to determine the cause.

The second analysis performed was to determine the radial distribution functions. Again, qualitative agreement with literature was found, but more work is needed to determine the uncertainties on the measured clustering coefficients, and to quantitatively compare the RDFs to theoretical models and literature values.

The Zugspitze experiment is not perfect. One issue that requires attention is the effect of the building on the flow. Risius et al. did measurements at a height of 6 metres above the roof of the Schneefernerhaus, and found that the flow is quite isotropic. The measurements presented in this thesis were conducted only 2 metres above the roof, and the results presented in chapter 6 warrant a closer look at the flow's isotropy. However, a simple solution might be to place the experiment either higher, or closer to the edge of the building. It could also be put on a sort of balcony, so that it extends away from the building.

The experiment has been built to be able to compensate for the mean wind. This is done by moving the LPT experiment over a set of rails using a linear motor. However, as was discussed in appendix D, it is difficult to deal with the vibrations this introduces. Furthermore, due to the large turbulence intensities (chapter 60 the large eddies sweep particles in and out of the LPT measurement volume about as quickly as the mean wind does. As a result the mean wind compensation is not expected to have large benefits, and therefore it should be carefully considered whether further development is worth the effort.

The Zugspitze experiment has been in development for about ten years now, and in the last four years it has been pushed to a level where it is finally 
able to produce scientific results. It is reasonable to expect that in a few years from now it can reliably produce measurements of the radial distribution function and the radial relative velocities. These results should prove useful to assess the accuracy of theoretical models for the RDF and the RRV at high Taylor Reynolds numbers. They could furthermore be used to improve the empirical models for cloud droplet growth and rain formation that are used in numerical weather prediction models.

There is evidence that both electric fields and droplet charge affect the collision-coalescence rate [1, 38, 49]-a topic that has not been discussed in this thesis. It would be interesting to add to the experiment an instrument that can measure these properties. 


\section{Appendix A}

\section{Integration of scattered irradiance}

A droplet's observed intensity $I_{O}$ is the total irradiance that is scattered by a droplet into a camera's aperture. It can be computed by evaluating equation (5.4), which is repeated here:

$$
I_{O}=\iint_{A} \mathrm{~d} A\left(\boldsymbol{x}_{a}^{\prime}\right) \iint_{4 \pi} w_{d} \mathrm{~d} \Omega\left(\hat{\boldsymbol{l}}^{\prime}\right) I_{s}\left(r^{\prime}, \theta^{\prime}\right) .
$$

The symbols used are defined in figure A.1. The first integral is over the camera's aperture $A$. If a diffuser is placed in the laser beam, light will be incident on the droplet from various directions; this is accounted for by the second integral. The diffuser's characteristic function $w_{d}\left(\hat{l}^{\prime}\right)$ is a twodimensional Gaussian that describes the diffuser's angular spread.

Equation (A.1) could be evaluated directly, but since it contains two twodimensional integrals, this could be rather time-consuming, even more so if one considers that it should be done for many droplet sizes and many angles $\theta$. Luckily though, two approximations can be made: 1 ) each of the two twodimensional integrals can be approximated by a one-dimensional integral, and 2) one of the resulting integrals can be evaluated immediately, yielding only a single integral. Let us now consider each of these approximations individually, and see why they can be done.

The first integral in equation A.1 reads

$$
\int_{A} \mathrm{~d} A\left(x_{a}^{\prime}\right),
$$




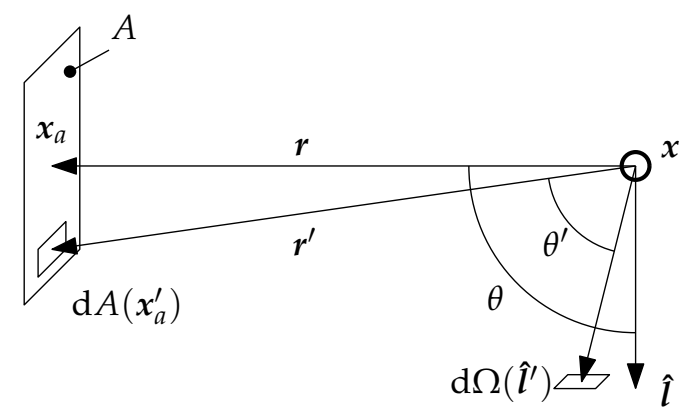

Figure A.1: Symbols used for solving equation A.1. A laser beam travelling in direction $\hat{l}$ is incident on a droplet at position $x$. Part of the light is scattered into the aperature, which has its centre at $\boldsymbol{x}_{a}$, and which for integration is split up into infinitesimals $\mathrm{d} A\left(x_{a}^{\prime}\right)$. If a diffuser is in use, light incident on the droplet will not come from a single direction $\hat{l}$, but from many directions $\hat{l}^{\prime}$, which for integration are again split into infinitesimals $\mathrm{d} \Omega\left(\hat{l}^{\prime}\right) . \quad r$ is the vector from the droplet's position to the aperture's centre, and $\theta$ is the angle between $\boldsymbol{r}$ and the laser beam's axis $\hat{\imath}$. The symbols with a prime have similar meanings, but relate to the infinitesimals.

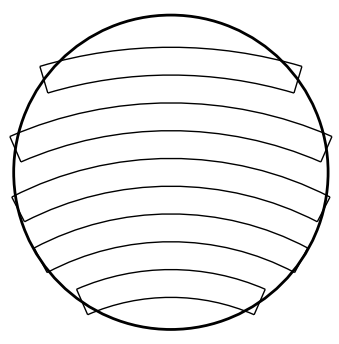

Strips of constant $\theta^{\prime}$
$2 r^{\prime} \sqrt{\alpha^{2}-\left(\theta^{\prime}-\theta\right)^{2}}$

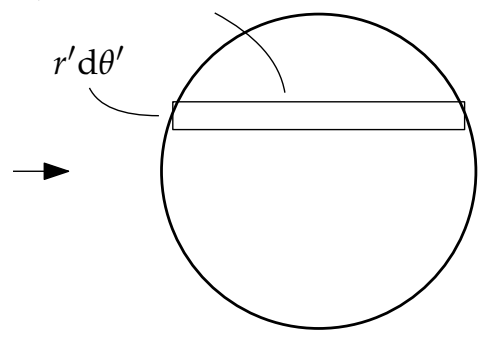

Approximated

Figure A.2: To integrate Lorentz-Mie scattering over the camera's aperture, it is natural to split the aperture in thin strips of constant $\theta^{\prime}$ (left). To make the integration itself easier, these strips can be approximated by thin rectangular strips, the length of which is easily calculated (right). The curvature of the strips in the left figure is strongly exaggerated. 
where the integrand, $I_{s}$, has been omitted for the sake of clarity. Note that the only variable that is relevant to computing $I_{S}$ (see equation (5.3)), is $\theta^{\prime}$; the other parameters, $r^{\prime}$ and $x$, are constant. It is therefore natural to express integral A.2 in spherical coordinates $r^{\prime}, \theta^{\prime}, \phi^{\prime}$, as follows:

$$
\int_{A} \mathrm{~d} A\left(x_{a}^{\prime}\right)=\int_{\theta-\alpha}^{\theta+\alpha} r^{\prime} \mathrm{d} \theta^{\prime} \int r^{\prime} \sin \left(\theta^{\prime}\right) \mathrm{d} \phi^{\prime},
$$

with $\theta$ approximately $30^{\circ}$, and $\alpha$ half the angle subtended by the aperture, so $\alpha \approx 0.5^{\circ}$. The integral over $\phi^{\prime}$ can be replaced by multiplication with a number, equal to the length of the thin strip of constant $\theta^{\prime}$ that is subtended by the aperture; see the left-hand side of figure A.2 for a graphical representation. Since the aperture is small and is sufficiently far away from both $\theta=0^{\circ}$ and $\theta=180^{\circ}$, the strips of constant $\theta^{\prime}$ can be approximated with thin rectangles, as is shown in the right of figure A.2. The length of these rectangles is given by $2 r^{\prime} \sqrt{\alpha^{2}-\left(\theta^{\prime}-\theta\right)}$, so the double integral can be written as:

$$
\int_{A} \mathrm{~d} A\left(x_{a}^{\prime}\right)=\int_{\theta-\alpha}^{\theta+\alpha} \mathrm{d} \theta^{\prime} r^{\prime 2} 2 \sqrt{\alpha^{2}-\left(\theta^{\prime}-\theta\right)^{2}} .
$$

For the sake of clarity it is beneficial to get rid of $\theta^{\prime}$ by substituting $\theta^{\prime}-\theta$ with $\xi_{1}$ :

$$
\begin{aligned}
\int_{A} \mathrm{~d} A\left(\boldsymbol{x}_{a}^{\prime}\right) & =\int_{-\alpha}^{\alpha} \mathrm{d} \xi_{1} r^{\prime 2} 2 \sqrt{\alpha^{2}-\xi_{1}^{2}} \\
& =\int_{-\alpha}^{\alpha} \mathrm{d} \xi_{1} f\left(\xi_{1}\right) .
\end{aligned}
$$

The remaining integral cannot be evaluated now, because the integrand, which was omitted for the sake of clarity, depends on $\theta^{\prime}$, which in turn depends $\xi_{1}$.

A similar approximation can be applied to the second integral in equation A.1. This integral reads

$$
\int_{4 \pi} w_{d} \mathrm{~d} \Omega_{D}\left(\hat{l}^{\prime}\right)
$$

where the integrand has again been omitted for the sake of clarity. Instead of integrating over a circular region, this integral is over all directions, i.e. over a solid angle of $4 \pi$. The diffuser's weight function is

$$
w_{d}\left(\hat{l}^{\prime}\right)=C_{d} \exp \left(-\frac{\left(\hat{l}^{\prime}-\hat{l}\right)^{2}}{2 \sigma_{d}^{2}}\right),
$$


with $\sigma_{d}$ the diffusion angle, and $C_{d}$ a constant so that $w_{d}$ integrates to 1 . The diffusion angle $\sigma_{d}$ is small, so $w_{d}$ is sharply peaked around the laser beam's axis $\hat{l}$. Because $w_{d}$ is so sharply peaked, this integral can be approximated in much the same way as the integral over the aperture. The result is:

$$
\begin{aligned}
\int_{4 \pi} w_{d} \mathrm{~d} \Omega_{D}\left(\hat{l}^{\prime}\right) & =\int_{-\infty}^{\infty} \mathrm{d} \xi_{2} \frac{1}{\sigma_{d} \sqrt{2 \pi}} \exp \left(-\frac{\xi_{2}^{2}}{2 \sigma_{d}^{2}}\right) \\
& =\int_{-\infty}^{\infty} \mathrm{d} \xi_{2} g\left(\xi_{2}\right) .
\end{aligned}
$$

Equations A.6 and A.10 can now be substituted in equation A.1, which gives:

$$
I_{O}=\int_{-\alpha}^{\alpha} \mathrm{d} \xi_{1} \int_{-\infty}^{\infty} \mathrm{d} \xi_{2} f\left(\xi_{1}\right) g\left(\xi_{2}\right) I_{s}\left(\theta^{\prime}\right)
$$

Note that $\theta^{\prime}$ has not formally been substituted here as was done before, but it can easily be seen that $\theta^{\prime}=\theta+\xi_{1}+\xi_{2}$. Whether the signs are plus or minus signs doesn't really matter, because $f$ and $g$ are even functions. Substituting $\xi_{1}+\xi_{2}=\xi$ finally yields:

$$
\begin{aligned}
I_{O} & =\int_{-\infty}^{\infty} \mathrm{d} \xi I_{s}(\theta+\xi) \int_{-\alpha}^{\alpha} \mathrm{d} \xi_{1} f\left(\xi_{1}\right) g\left(\xi-\xi_{2}\right) \\
& =\int_{-\infty}^{\infty} \mathrm{d} \xi I_{S}(\theta+\xi) h(\xi) .
\end{aligned}
$$

The function $h$ depends on the droplet-aperture distance $r$, the aperture's subtended half-angle $\alpha$, and the diffuser's characteristic function $w_{d}$, and, if these parameters do not change, needs to be computed only once. 


\section{Appendix B}

\section{Bayesian inference of droplet size}

This appendix summarises the results of a hurried attempt at using Bayesian inference to determine droplet sizes. The potential advantage of this method, is that it correctly deals with oscillations in the scattered intensity.

\section{B.1 Bayesian inference}

Bayes' theorem (e.g. [40]) states that

$$
P(A \mid B)=\frac{P(B \mid A) P(A)}{P(B)},
$$

with

- $P(A)$ and $P(B)$ the probabilities that $A$ and $B$ are true, respectively;

- $P(A \mid B)$ is the probability that $A$ is true given that $B$ is true; and

- $P(B \mid A)$ is the probability that $B$ is true given that $A$ is true.

In the Bayesian interpretation of probability, these probabilities are degrees of belief, i.e. $P(A)$ is the degree of belief that $A$ is true. In the context of an experiment, $P(A)$ is called the prior, that is the degree of belief that $A$ is true, prior to having seen the evidence $B$. That makes $P(A \mid B)$ the posterior, and Bayesian inference is the process of determining it. 


\section{B.2 Bayesian droplet sizing}

Let $I$ be a vector of intensities observed along a trajectory. Bayes' theorem states that the probability that these specific intensities are caused by a droplet of diameter $d_{p}$, is

$$
P\left(d_{p} \mid \boldsymbol{I}\right)=\frac{P\left(\boldsymbol{I} \mid d_{p}\right) P\left(d_{p}\right)}{P(\boldsymbol{I})} .
$$

The prior $P\left(d_{p}\right)$ can be taken to be constant, i.e. all droplet sizes are equally probable. The denominator is a constant of which the exact value doesn't matter, because the posterior can be normalised such that it integrates to 1 . Equation (B.2) then becomes

$$
P\left(d_{p} \mid \boldsymbol{I}\right) \propto P\left(\boldsymbol{I} \mid d_{p}\right) .
$$

The expected value operator $E[X]$ is:

$$
E[X]=\int_{0}^{\infty} X P\left(\boldsymbol{I} \mid d_{p}\right) \mathrm{d} d_{p}=\frac{\int_{0}^{\infty} X P\left(d_{p} \mid \boldsymbol{I}\right) \mathrm{d} d_{p}}{\int_{0}^{\infty} P\left(d_{p} \mid \boldsymbol{I}\right) \mathrm{d} d_{p}} .
$$

With this the droplet size $\mu_{d_{p}}$ and uncertainty $\sigma_{d_{p}}$ can be computed as follows: 1

$$
\begin{gathered}
\mu_{d_{p}}=E\left[d_{p}\right], \\
\sigma_{d_{p}}^{2}=E\left[\left(d_{p}-\mu_{d_{p}}\right)^{2}\right],
\end{gathered}
$$

which are the usual definitions of the mean and the standard deviation.

Before equations (B.5) and (B.6) can be put to use, an expression for $P\left(\boldsymbol{I} \mid d_{p}\right)$ is needed. If it is assumed that

- observed intensities are independent, and normally distributed around their expected values; and

- expected intensities can be computed with equation (5.5),

then

$$
P\left(\boldsymbol{I} \mid d_{p}\right)=\prod_{j} \prod_{k} P_{N}\left(I_{j k} \mid \mu_{j k}, \sigma_{j k}\right),
$$

${ }^{1}$ In other chapters the droplet size is simply $d_{p}$. In this appendix that is used as a variable of integration, and instead $\mu_{d_{p}}$ is used as the measured droplet size. 
with $P_{N}$ the normal PDF, $\mu_{j k}$ the expected intensity, and $\sigma_{j k}$ the intensity standard deviation. Here, and in the rest of this appendix, the following subscripts are used: $i$ for a droplet trajectory, $j$ for a point along a trajectory, and $k$ for a camera. Depending on context some subscripts might be omitted; in this section, for instance, a single droplet trajectory is considered, so the index $i$ is omitted.

Finally, an expression for $\sigma_{j k}$ is needed. Here it is assumed that

$$
\sigma_{j k}=c_{0} \mu_{j k}^{c_{1}}
$$

with $c_{0}$ and $c_{1}$ model parameters that are to be determined. Dr. Moláček suggested that the standard deviation should also depend on distance $r-r_{0}$ to the camera's focus plane:

$$
\sigma_{j k}=c_{0} \mu_{j k}^{c_{1}}\left[1+c_{2}\left(r-r_{0}\right)^{2}\right]^{c_{3}},
$$

with $r_{0}$ and $c_{3}$ additional model parameters.

\section{B.3 Model parameters}

\section{B.3.1 Estimate from histograms}

Initial estimates for the model parameters are obtained from histograms of the error in the measured intensities. The true intensities are not known, so the errors must be approximated. To do so, it is assumed that neighbouring true intensities are approximately equal, so that the error is

$$
e_{i j k} \approx I_{i j k}-I_{i, j-1, k} \text {. }
$$

If the intensities come from normal distributions, then so do the errors $e$. Technically it is not possible to compute the standard deviation of $e$, since each of these comes from a different normal distribution. However, if LorentzMie scattering and variation due to the laser beam profile are ignored, then $\mu_{i j k}=\mu_{i k}$ and $\sigma_{i j k}=\sigma_{i k}$, and

$$
\sigma_{i k}^{2}=\frac{1}{2}\left\langle e_{i j k}^{2}\right\rangle_{j}
$$

with the average running over each point $j$ in the trajectory. These are compared to the trajectory-averaged intensities

$$
\mu_{i k}=\left\langle I_{i j k}\right\rangle_{j} .
$$


Instead of the average squared error $\left\langle e^{2}\right\rangle$, the variance of $I_{i j k}$ could have been used. The former, however, acknowledges that the intensity is not constant throughout a trajectory, and should give a slightly smaller value for $\sigma_{i k}$ than $\operatorname{Var}\left(I_{i j k}\right)_{j}$ does. To get the distribution of relative errors, $e_{i j k}$ could also be compared to $m_{i j k}=I_{i j k}+I_{i, j-1, k}$, i.e. $e_{i j k} / m_{i j k}$. This would give more accurate results, but it might be difficult to relate the distribution of these relative errors to $\sigma_{i k}$.

2D histograms of $\left(\ln \left(\mu_{i k}\right), \sigma_{i k} / \mu_{i k}\right)$ are plotted in figure B.1 for two cases. Case \#1 is a low seeding density case (experiment 146 on 25-9-2019), and case \#2 is a high seeding density case (experiment 367 on the same day). The relative error is approximately 0.1 , although it seems to differ slightly between the cases. The model parameters should therefore be determined again for each experiment. All histograms show an increase in relative error for fainter droplets. This suggests that $c_{1}<1$. The histograms for case \#1 also show an increase for brighter droplets, which suggests that $c_{1}>1$.

Histograms were also made for different $z$ slices, but no notable difference was seen.

\section{B.3.2 Maximum likelihood estimation}

In this section the model parameters are determined using maximum likelihood estimation, or MLE. This will be done for each camera individually, so $k$ is constant and will be omitted. The likelihood $\mathcal{L}$ is given by

$$
\mathcal{L}\left(c_{0}, c_{1}, \mu_{1}, \mu_{2}, \ldots, \mu_{N}\right)=\prod_{i} \prod_{j} P_{N}\left(I_{i j} \mid \mu_{i j}, \sigma_{i j}^{2}\right) .
$$

Lorentz-Mie scattering is ignored, but the laser beam profile is taken into account. The mean and standard deviation of the normal distributions are then given by

$$
\begin{gathered}
\mu_{i j k}=I_{i j}^{(0)} \cdot \mu_{i k}, \\
\sigma_{i j k}=c_{0} \mu_{j k^{\prime}}^{c_{1}}
\end{gathered}
$$

with $I_{i j}^{(0)}$ the laser beam intensity at the droplet's position $j$, and $\sigma_{i j k}$ conform equation (B.8). By maximising $\mathcal{L}$, values for $c_{0}$ and $c_{1}$ can be found. As a side-effect, optimal values for $\mu_{1}$ etc. will also be found, but these will be discarded. In practice it is easier and numerically more stable to work with 


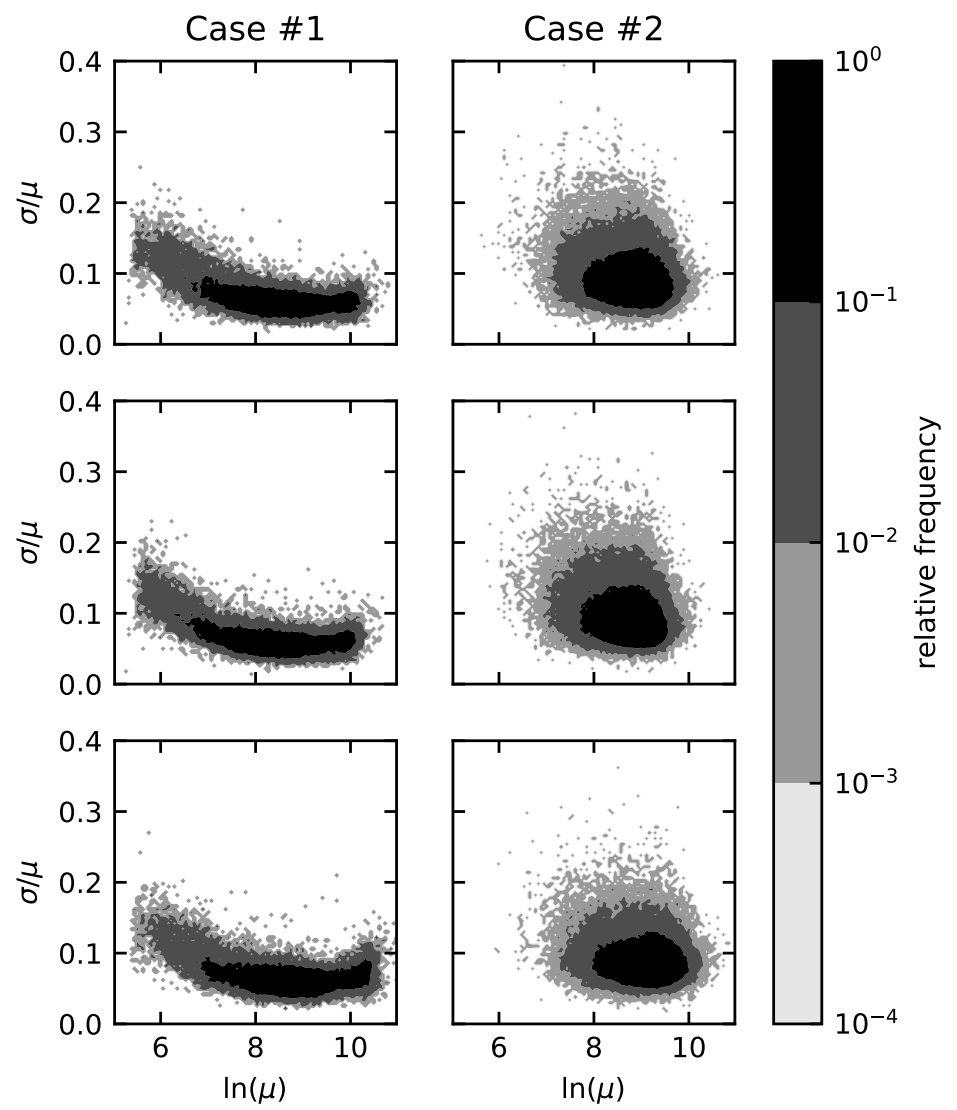

Figure B.1: Histograms of relative errors, computed per trajectory. The rows correspond to the three cameras, while the columns respond to cases. Case \#1 has a low seeding density (experiment 146 on 25-9-2019); case \#2 has a high seeding density (experiment 367 on the same day). 
the log-likelihood:

$$
\ell\left(c_{0}, c_{1}, \mu_{1}, \mu_{2}, \ldots, \mu_{N}\right)=\ln (\mathcal{L})=\sum_{i} \sum_{j} \ln \left[P_{N}\left(I_{i j} \mid \mu_{i j}, \sigma_{i j}\right)\right] .
$$

The log-likelihood is optimised for all experiments on 25-9-2019 using a Python code. The SciPy package comes with a number of optimisation routines; here the Newton conjugate gradient method ("newton-cg") is used, because it is suitable for large problems, i.e. problems with a large Hessian. The problem at hand is large: case \#2 has 1117329 trajectories, for each of which the mean intensity will be found; the entire Hessian is a $10^{6} \times 10^{6}$ matrix. The gradient and Hessian of (B.16) are specified explicitly, and are verified by comparing them to numerical derivatives of (B.16). The Hessian is sparse, so computing it explicitly is much faster than numerical derivation. Another advantage of using explicit derivatives, is that they are more accurate than numerical approximations, and hence the optimisation is more accurate. Finally, to speed things up, the Numba package is used, which is essentially a just-in-time compiler for certain kinds of Python code.

The MLE is run per experiment and per camera, for all experiments on 25-9-2019. To see if there is any dependence on initial guess for $c_{0}$ and $c_{1}$, all combinations of $c_{0} \in\{0.3,0.4,0.5\}$ and $c_{1} \in\{0.8,0.9,1.0\}$ are tried. Histograms of all optimised $c_{0}$ and $c_{1}$ are shown in figure B.2 The optimal parameters appear in 9 groups, each of which corresponds to an initial guess. The optimal parameters should not depend on the initial guess; that they do, suggests that the optimisation ends up in a local maximum, or does not converge at all.

The author speculates that non-convergence is likely. To see why, consider the form of the gradient:

$$
\nabla \ell=\left(\frac{\partial \ell}{\partial c_{0}}, \frac{\partial \ell}{\partial c_{1}}, \frac{\partial \ell}{\partial \mu_{1}}, \frac{\partial \ell}{\partial \mu_{2}}, \ldots, \frac{\partial \ell}{\partial \mu_{N}},\right) .
$$

The first two terms have contributions from all trajectories, while the rest only has contributions from single trajectories. The first two terms are therefore much larger than the rest. This might pose a problem for the optimisation routines used here. A solution could be to split the problem in two, i.e. to alternate between optimising $c_{0}$ and $c_{1}$, and optimising the $\mu_{i}$. The theoretical basis for this is the generalised expectation-maximisation algorithm [26, 43]. 


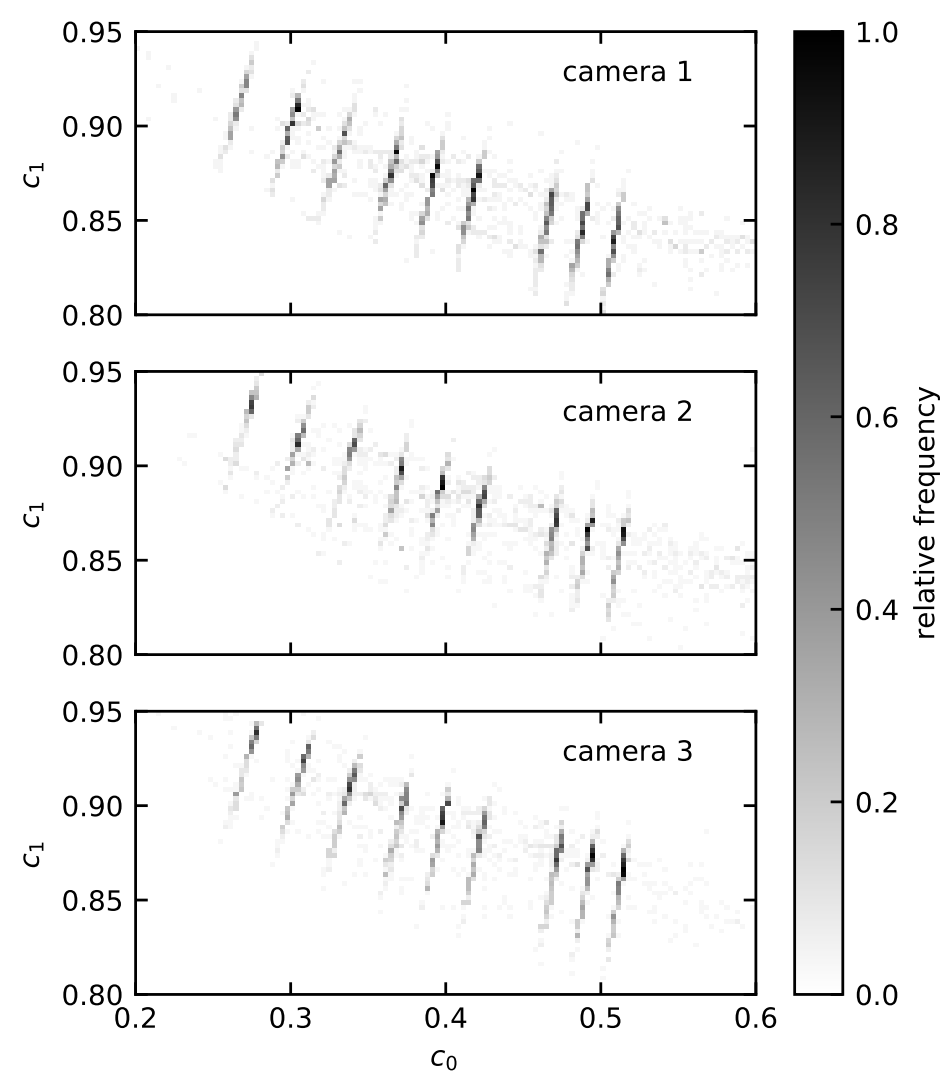

Figure B.2: Histograms of model parameters, computed for all particle tracking experiments on 25-9-2019. 


\section{B.4 Droplet sizing}

Droplet sizes can be determined by evaluating equation (B.5). To do so, the integrals in equation (B.4) are first discretised, i.e. replaced with a sum over a finite number of droplet sizes. Then, a lookup table of expected intensities $\mu$ is computed using equation (5.5) for various droplet sizes, for various scattering angles, and for each camera. Here 9901 droplet sizes in the range $1 \mu \mathrm{m}$ to $100 \mu \mathrm{m}$, and $\sim 1700$ central scattering angles in the range $20^{\circ}$ to $40^{\circ}$ are used. Then, for each trajectory, the following steps are taken:

1 . The central scattering angles $\theta_{j k}$ are computed, and the corresponding expected intensities $\mu_{j k}$ are looked up using the table created earlier.

2. The laser beam intensity $I_{j}^{(0)}$ at each point along the trajectory is looked up, and is factored into the expected intensities.

3. The intensity standard deviations $\sigma_{j k}$ are computed using equation (B.8).

4. The logarithm of equation (B.7) is evaluated.

5. Equations (B.5) and (B.6) are evaluated.

This method is applied to case \#2. The resulting droplet size histogram is shown in the top graph of figure B.3. The bottom graph shows CDFs of the relative droplet size uncertainty for three ranges of droplet sizes. As can be seen, for only $1 \%$ of all trajectories, the relative uncertainty exceeds 0.08 . This is twice as accurate as the droplet sizing method presented in chapter 5 (see figure 5.7).

The CDFs also show that for half of all trajectories the relative uncertainty is $<0.01$. This is unrealistic, given that this method depends very sensitively on accurate knowledge of amongst others the direction of the laser beam, the aperture sizes, and the camera sensitivity (i.e. counts-per-photon).

To better understand how this method works, PDFs computed with equation (B.7) using data from the field experiment, are plotted in figure B.4 for three different trajectories. The product in equation (B.7) has not been applied yet, i.e. these are per-camera droplet size PDFs. Interpretation of these plots is left for later work.

\section{B.5 Conclusion}

While Bayesian-inference droplet sizing has the potential to deal with intensity oscillations due to Lorentz-Mie scattering, the method has a big downside: 

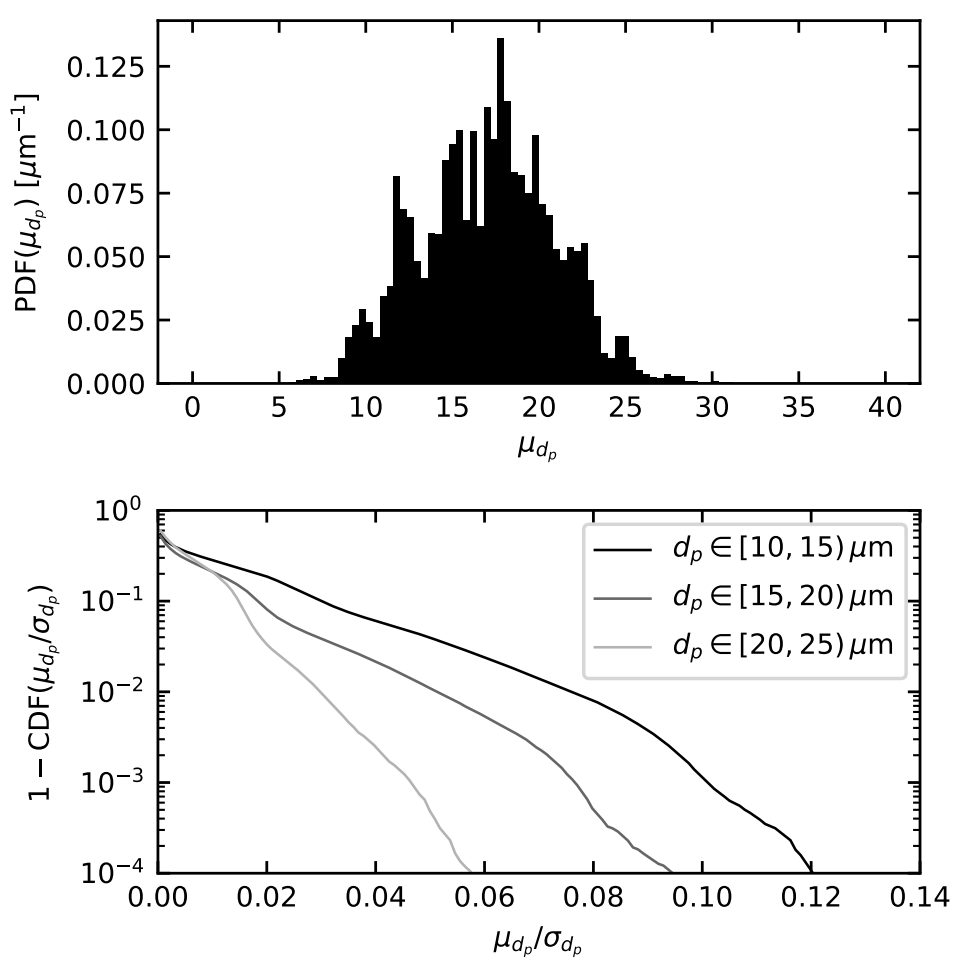

Figure B.3: Top: histogram of droplet sizes for case \#2. Bottom: CDFs of relative droplet size uncertainty for three ranges of droplet sizes. 

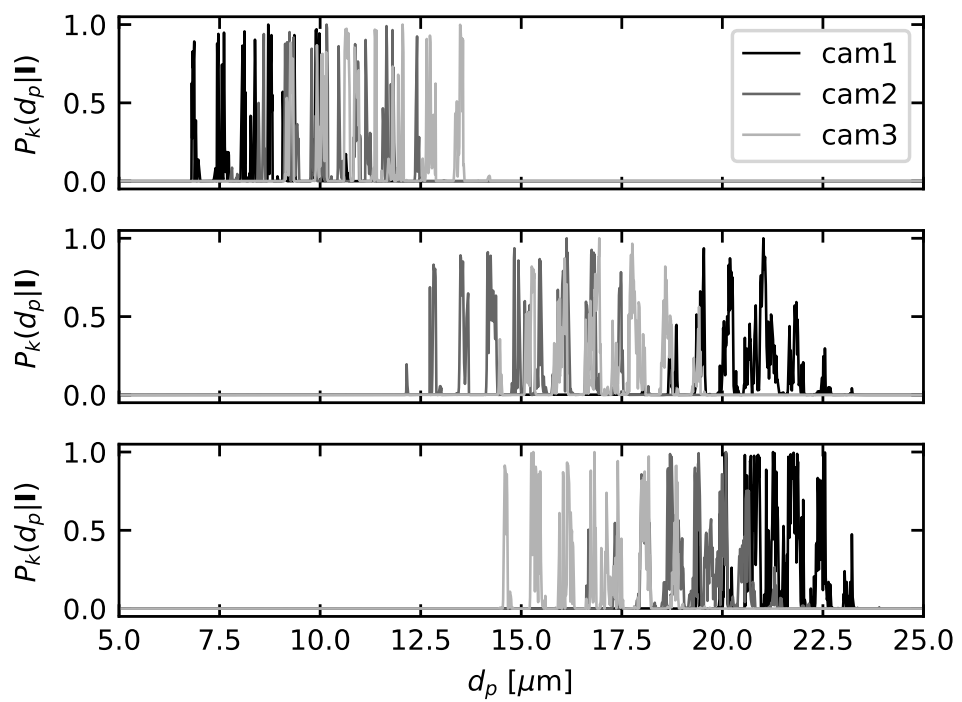

Figure B.4: PDFs computed with equation (B.7) for three different trajectories, using data from field experiments. These PDFs are before multiplication over the cameras, and are scaled so that the maximum of each is 1 . 
it is slow. With the discretisation chosen here, for a 20-point trajectory a total of $3 \times 20 \times 9901 \approx 6 \times 10^{5}$ normal distributions must be evaluated. The computational cost is hence much larger than that of the method presented in chapter 5. which is essentially taking a square root. It must be questioned whether it is worth the extra effort.

The first step in further developing this method, should be to determine various properties of the experimental setup more accurately. In particular the laser beam angle, the aperture diameters, and the sensitivity of the cameras (counts-per-photon) should be known accurately.

Furthermore, a more accurate model for the intensity uncertainty is needed, as this directly influences the computed droplet size uncertainties cf. equation B.6 Depending on the model, a better strategy to optimise its parameters (section B.3) may also be needed. 


\section{Appendix C}

\section{Calibration of the FMAG aerosol generator}

\section{C.1 The FMAG aerosol generator}

Droplets are produced with the TSI FMAG Model 1520. The principle of this aerosol generator was first published in an article titled: "Generation of monodisperse aerosols by combining aerodynamic flow-focusing and mechanical perturbation" [15]. Flow focusing refers to the use of two concentric nozzles, the inner of which ejects liquid, and the outer ejects gas. The outer gas jet accelerates the inner fluid jet, thinning it, and thereby allowing it to produce droplets that are much smaller than the orifice from which it came. The diameter $d_{j}$ of the inner jet is given by

$$
d_{j}=\left(\frac{8 \rho_{l}}{\pi^{2} \Delta P_{g}}\right)^{1 / 4} Q^{1 / 2},
$$

with $\rho_{l}$ the liquid density, $\Delta P_{g}$ the gas pressure drop, and $Q$ the liquid volume flow. The FMAG uses a piezo-electric element to impose a periodic perturbation on the liquid jet. The perturbation grows due to the Rayleigh instability, and causes the jet to break up into droplets with a diameter $d_{p}$ given by

$$
d_{p}=\left(\frac{6 Q}{\pi f}\right)^{1 / 3}
$$




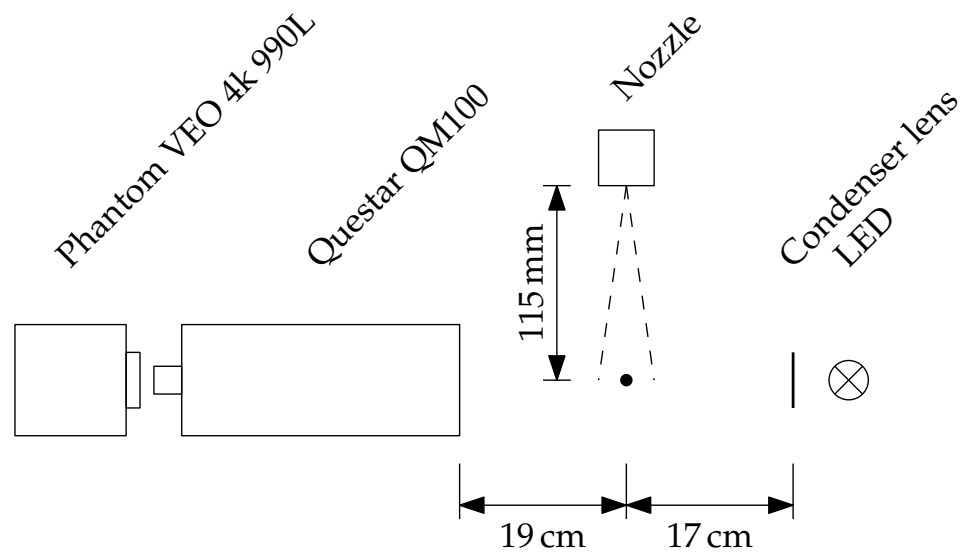

Figure C.1: Diagram of the shadowgraphy setup.

with $f$ the piezo driving frequency. The wavelength $\lambda$ of the perturbation is

$$
\lambda=\frac{4 Q}{\pi d_{j}^{2} f} .
$$

For the jet to break up into monodisperse droplets, $\lambda$ should ideally be $4.5 d_{j}$, but values between $3.5 d_{j}$ and $7 d_{j}$ are known to work as well.

The the gas pressure drop $\Delta P_{g}$, the liquid volume flow $Q$, and the driving frequency $f$ can be set using knobs on the device. A combination of these parameters constitutes a recipe. The aim of this chapter is to find recipes that can be used to reliably produce monodisperse droplet size distributions. To this end a shadowgraph is first built, calibrated, and tested. The shadowgraph is used to measure the droplet size distributions resulting from various recipes under test, and the recipes are iteratively improved upon.

\section{C.2 Shadowgraphy}

\section{C.2.1 Setup}

Out of the box the FMAG can produce droplets ranging from $17 \mu \mathrm{m}$ to $39 \mu \mathrm{m}$ in diameter, but by taking the nozzle out and inverting it, it can produce droplets of up to $150 \mu \mathrm{m}$ in diameter. Droplets larger than $72 \mu \mathrm{m}$ were measured with a commercial PDA probe (Artium PDI-FPDR) at Zugspitze, so the inverted 


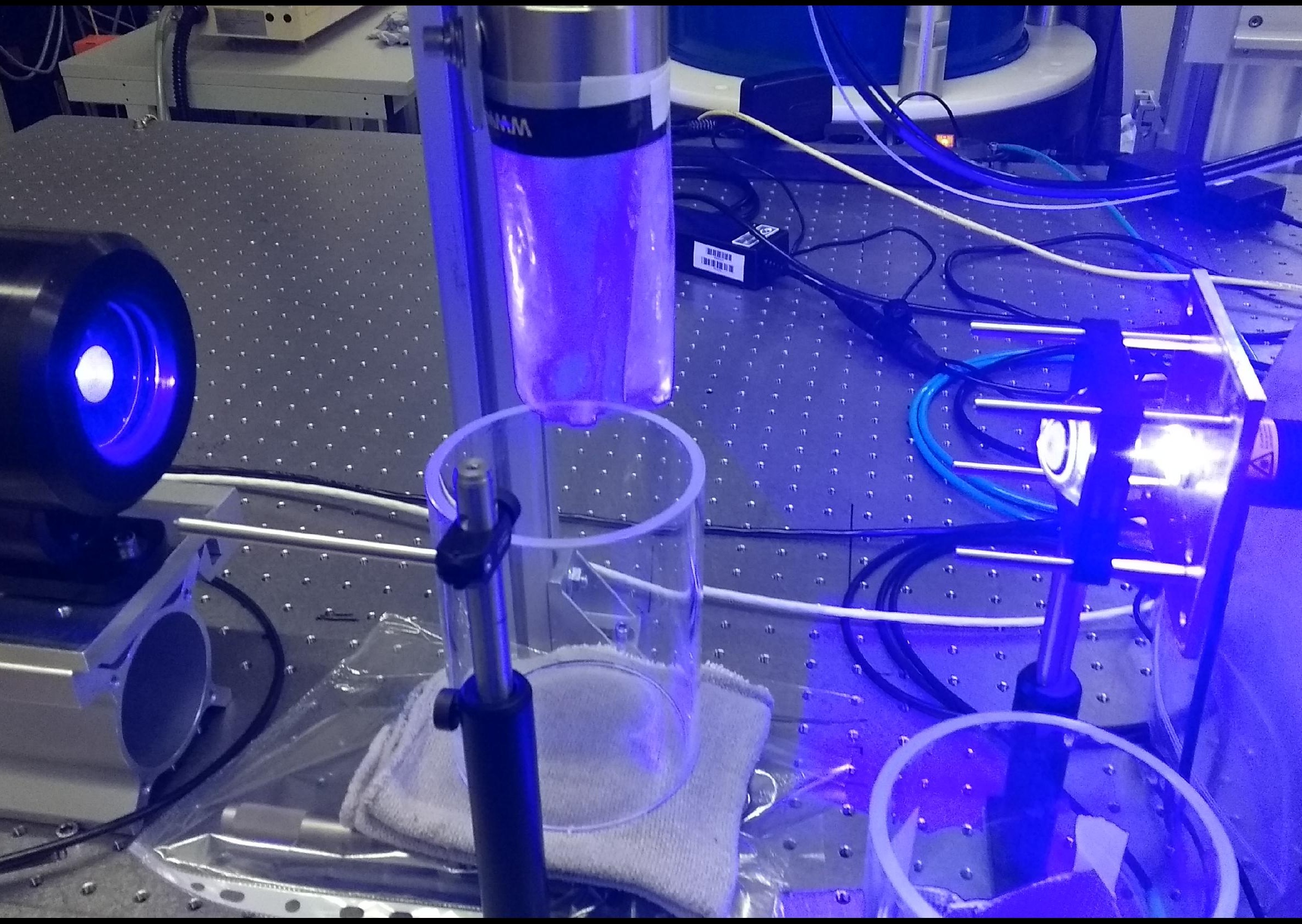




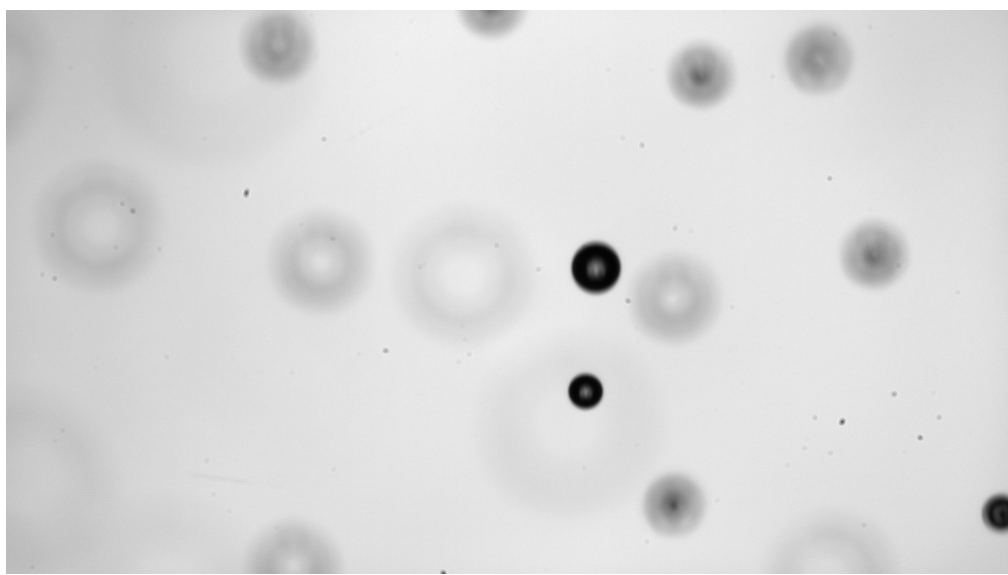

Figure C.2: A typical shadowgraphy image.

configuration is used to generate droplets. The FMAG's manual does not provide values for $Q, f$, and $\Delta P_{g}$ for this configuration, so an experiment is needed to find the right values. To this end a shadowgraph was built using the following components:

- Thorlabs M455L3 high power LED, $455 \mathrm{~nm},>900 \mathrm{~mW}$

- Thorlabs ACL50832U-A condenser lens, $f=32 \mathrm{~mm}, 2$ in diam.

- Questar QM100 Maksutov-Cassegrain long distance miscroscope objective

- Phantom VEO 4K 990L high speed camera, 4096 px × 2304 px

A diagram of the setup is shown in figure C.1. The shadowgraph is calibrated using calibration mask with a $0.2 \mathrm{~mm} \times 0.2 \mathrm{~mm}$ marker grid, and is found to have a magnification of $0.49 \mu \mathrm{m} \mathrm{px}^{-1}$, so its field of view is $2.0 \mathrm{~mm} \times 1.1 \mathrm{~mm}$. To minimise motion blur the exposure time was set to $5 \mu \mathrm{s}$, which is the shortest the camera can do. A typical shadowgraphy image is shown in figure C.2

\section{C.2.2 Data analysis}

The shadowgraphy images are analysed using a robust method developed by Legrand et al. [36], that works as follows: 
1. Divide the image by a background (white) image, to correct for inhomogeneities in the illumination. The pixel values are now in the range $[0,1]$.

2. Identify particle shadows by looking for groups of pixels that are sufficiently dark, i.e. at least 400 pixels with values $<0.5$.

3. For each shadow:

(a) Count the number of pixels that are $<0.5$, and call this $N_{1}$. Compute an approximate diameter $D_{x}=2 \sqrt{N_{1} / \pi}$.

(b) Isolate the shadow: cut out a square region centred on the shadow, that is $2.25 D_{x}$ on each side.

(c) Rescale the shadow's pixel values, so that they are in the range $[0,1]$.

(d) Count the number of pixel values $<0.7$ and call this $N_{0.7}$. Compute an approximate diameter $D_{0.7}=2 \sqrt{N_{0.7} / \pi}$.

(e) Count the number of pixel values $<0.3$ and call this $N_{0.3}$. Compute an approximate diameter $D_{0.3}=2 \sqrt{N_{0.3} / \pi}$.

(f) Reject the shadow if $D_{0.7} / D_{0.3}>1.2$; this indicates the shadow is too much out of focus. Legrand et al. [36] use a less strict threshold of 2.5 .

(g) Compute the particle diameter from $D_{0.3}$ and $D_{0.7}$. This boils down to evaluating a few polynomial functions; for details the reader is referred to Legrand et al. [36].

This method was implemented in Python using OpenCV, and was verified using shadowgraphy images taken of $80 \mu \mathrm{m}$ polystyrene beads (Dynoseeds TS 80-50).

\section{C.3 Results}

The equations and conditions given in section C.1 were used to generate an initial set of recipes for various droplet sizes. Each of these recipes was tested using the shadowgraph. In many cases the droplet size distribution was not monodisperse: some recipes yielded a very broad droplet size distribution, others yielded multiple peaks, and yet others did not yield the target droplet size at all. In such cases the recipe was tweaked until it gave a satisfactory 
Table C.1: FMAG recipes for various droplet diameters.

\begin{tabular}{cccc}
\hline$d_{p}[\mu \mathrm{m}]$ & $Q\left[\mathrm{mlh}^{-1}\right]$ & $f[\mathrm{kHz}]$ & $\Delta P_{g}[\mathrm{psi}]$ \\
\hline 20 & 2.0 & 132.6 & 2.20 \\
30 & 3.5 & 70.1 & 1.50 \\
40 & 6.0 & 51.3 & 1.50 \\
50 & 9.5 & 39.0 & 1.50 \\
60 & 15.0 & 36.8 & 1.90 \\
70 & 12.9 & 20.0 & 0.75 \\
80 & 19.3 & 20.0 & 0.90 \\
90 & 27.0 & 19.8 & 1.30 \\
100 & 27.5 & 14.6 & 0.80 \\
110 & 42.0 & 16.8 & 1.30 \\
120 & 50.0 & 15.4 & 1.20 \\
\hline
\end{tabular}

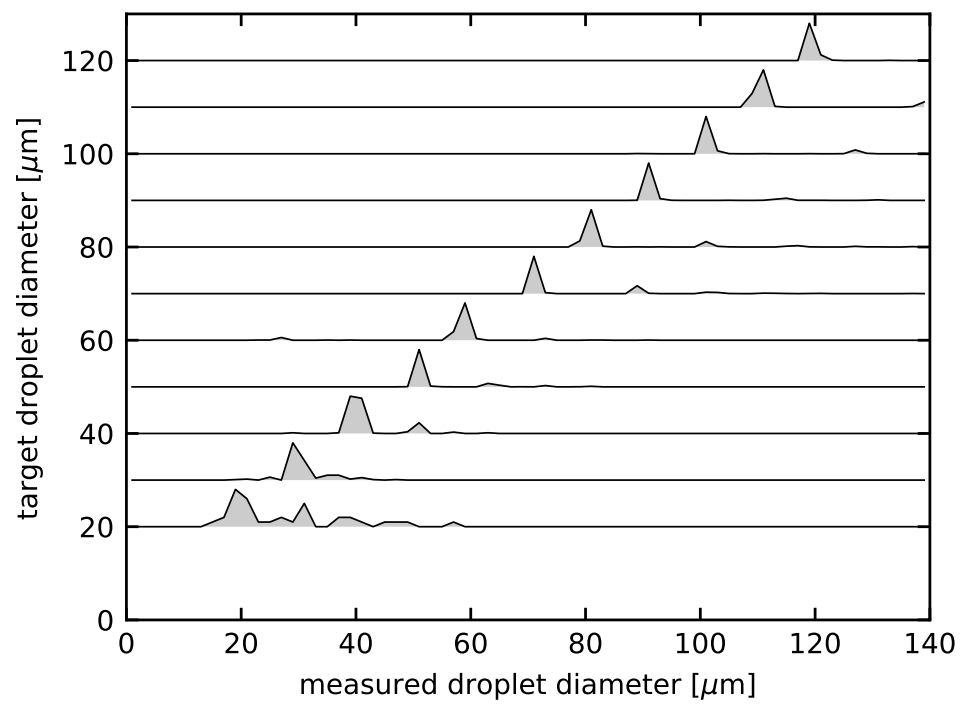

Figure C.3: Distributions of droplet sizes generated with the recipes listed in table C.1 The vertical offsets correspond to the target droplet diameter. 
droplet size distribution. The resulting recipes were tested at least twice, to ensure repeatability. Table C.1 lists the final recipes. For each size the corresponding droplet size distribution is shown in figure C.3

\section{C.4 Notes}

- This is by far not an exhaustive performance test of the FMAG aerosol generator, and it was never intended to be. The FMAG was only calibrated in as far as was needed for it to be usable as a calibrated droplet source.

- A pulsed laser would have provided more light than the LED does, and would also have eliminated all motion blur. 
128 APPENDIX C. CALIBRATION OF THE FMAG AEROSOL GENERATOR 


\section{Appendix D}

\section{Mean-wind compensation}

The Schneefernerhaus and wind tunnel experiments have in common that there is a mean wind. Depending on the quantity of interest this mean wind can be a problem. If one wishes to observe two-particle dispersion in the Richardson-Obukhov regime [56], one needs to be able to see the same particle pair for an extended period of time. The mean wind prevents this, because it will blow the particle pair out of the measurement volume before Richardson-Obukhov dispersion sets in.

Whether the presence of a mean wind is preferred or not also depends on the experimental technique that is used. For some experimental techniques (e.g. hot-wire anemometry and laser-Doppler velocimetry) the presence of a mean wind is vital. For other experimental techniques it either doesn't matter (e.g. sonic anemometry, PIV), or can even be detrimental (e.g. particle tracking). In the case of particle tracking, the mean wind is a complicating factor for the following two reasons:

- The mean wind causes a larger particle displacement from frame to frame. As a result, particle tracking codes need to use a larger search radius to track a particle from one frame to the next, in particular for new particles, for which no mean velocity is known yet. For high seeding densities this might lead to many false trajectories. It can be remedied by increasing the frame rate, which comes at the expense of shorter experiments and/or more expensive cameras and lasers.

- The mean wind causes particles to remain in the measurement volume for a shorter time. This results in shorter particle trajectories, which means that less smoothing can be done. Smoothing particle trajectories 
is beneficial when computing particle velocities and accelerations, as these temporal derivatives amplify the particle position noise.

To compensate for the mean wind at the Schneefernerhaus the seesaw was built. The seesaw consists of a pair of rails over which the particle tracking setup slides. It is introduced in the next section. The sliding introduces vibrations, that are detrimental to the particle tracking, and therefore must be damped. This is further discussed in section D.2.

\section{D.1 Seesaw}

This section will be submitted as part of an article to Review of Scientific Instruments.

The seesaw allows us to compensate for the mean wind. It supports the rails on which the table slides; see figure 3.1. It has been designed for velocities up to $7.5 \mathrm{~m} \mathrm{~s}^{-1}$ and accelerations up to $28 \mathrm{~m} \mathrm{~s}^{-2}$. It also withstands emergency stops, which occur for instance if the linear motor fails and the table runs into the endstops; in such events the deceleration can reach up to $230 \mathrm{~m} \mathrm{~s}^{-2}$.

The highest frame rate we typically record at is $10 \mathrm{kHz}$; at this frame rate we can record up to $1.6 \mathrm{~s}$ of data before exhausting the camera buffers. During this time the table should be moving at a constant speed. We normally limit the acceleration and deceleration to $10 \mathrm{~m} \mathrm{~s}^{-2}$, to prevent the box from shaking too much. Given that the maximum travel is $5.3 \mathrm{~m}$, this gives a maximum constant velocity of $2.8 \mathrm{~m} \mathrm{~s}^{-1}$, which is in our experience sufficiently high most of the time.

Risius [51] found that the inclination of the mean wind varies between $-15^{\circ}$ and $15^{\circ}$. The seesaw is built to account for this: using a hand crank it can pivot around an axis close to the centre of the rail. To give the structure sufficient stiffness two lockable, telescoping cylinders have been added on either side of the axis.

The seesaw and its supporting structure weigh approximately $4400 \mathrm{~kg}$. The table and camera box may weigh up to $300 \mathrm{~kg}$. If, due to motor or motor controller failure, they run into the shock absorbers, this could exert a force of up to $140 \mathrm{kN}$. To account for these forces, mounting points poured in concrete have been installed on the roof of the Schneefernerhaus.

A sliding table is mounted on a pair of rails on the seesaw. It is driven with a linear motor that is part of the Bosch-Rexroth IndraDyn L series, a series of industrial three-phase synchronous motors. In particular we are using a kit motor, which means that the stator and rotor are delivered separately, and the 
buyer is responsible for making a complete motor out of them. In our case the stator consists of plates of permanent magnets that are mounted on the seesaw in between the rails. The rotor consists of two sets of electromagnets that are mounted on the bottom side of the table.

The actual position of the table is measured using the Bosch-Rexroth Integrated Measuring System (IMS), which integrates a magnetic grating with a period of $40 \mu \mathrm{m}$ in the side of one of the rails, and an inductive sensor head in one of the carriages. Initially, a more precise optical grating was used to measure position, however during the environmental conditions most suitable for our experiments it was found to be extremely unreliable. Cloud droplets would stick to the grating and collect into a larger drop as the sensor head would travel over it, disrupting the functionality of the optical sensor head. The magnetic system on the other hand is completely unaffected by the presence of water on the grating.

The motor is controlled by a Bosch-Rexroth IndraDrive $C$ series controller, which is installed in a cabinet in the laboratory, two floors below the experiment. At its heart it has a set of cascaded PID controllers that control the motor position by setting the motor current, taking maximum velocity, acceleration, and jerk (third derivative of the position with respect to time) into account. The IndraDrive controller allows automated, real-time control, either by loading a motion control programme into it, or through a real-time bus such as CANopen, but at present we control it by hand, using software provided by Bosch-Rexroth.

\section{D.2 Vibration damping}

The seesaw introduces vibrations via two mechanisms:

- The linear motor stator has a magnetic pole every $2.5 \mathrm{~cm}$. As the table travels the rotor repeatedly aligns and misaligns with the stator, and as a result its attraction to the stator becomes a function of position. This effect is called cogging torque, and as a result, the table vibrates as it travels. The motor controller has functionality to compensate the cogging torque, but it proved difficult to tune this correctly, and therefore wasn't used here.

- The camera box and its mounting hardware form a forced mass-spring system, with the forcing coming from the motion of the table. While this mass-spring system helps to damp the vibrations due to cogging 
Table D.1: RMS acceleration of the prototype seesaw's table for various velocities. $x$ is along the direction of travel; $z$ is parallel to gravity.

\begin{tabular}{clll}
\hline Velocity & \multicolumn{3}{c}{ RMS acceleration $\left[\mathrm{m} \mathrm{s}^{-2}\right]$} \\
{$\left[\mathrm{m} \mathrm{s}^{-1}\right]$} & $x$ & $y$ & $z$ \\
\hline Motor off & 0.005 & 0.005 & 0.005 \\
Standstill & 0.040 & 0.040 & 0.025 \\
0.5 & 0.5 & 0.1 & 0.2 \\
1.0 & 1.2 & 0.3 & 0.3 \\
1.5 & 1.6 & 0.2 & 0.4 \\
2.0 & 2.2 & 0.3 & 0.5 \\
2.5 & 2.8 & 0.4 & 0.5 \\
3.0 & 3.4 & 0.4 & 0.5 \\
\hline
\end{tabular}

torque, it may start oscillating due to large-scale motions, i.e. when the table is accelerated from standstill to the mean wind speed.

In the following subsections the vibrations are quantified first. Then two different approaches are explored to damp the vibrations: a passive approach, in which the camera box becomes part of a mass-spring system, and an active approach, in which the stiffness of the mass-spring system is increased during acceleration and deceleration of the box.

All experiments are done using a prototype of the seesaw in a laboratory in Göttingen. The prototype has shorter rails than the seesaw itself; it allows for $2250 \mathrm{~mm}$ of travel.

\section{D.2.1 Table vibration}

The vibrations of the table are measured using a Silicon Designs SDI-2470-005 triaxial acceleratometer. It is mounted such that its $x$-direction is along the direction of travel and $z$ is vertical. It is connected to a LabJack T7 Pro, which samples the accelerometer's output at $1 \mathrm{kHz}$.

The motor is controlled using the Bosch-Rexroth software running in easy startup mode. In this mode, the motor can only execute simple motion profiles. In particular, velocity changes follow a linear profile (i.e. constant acceleration), and jerk limiting (jerk $=\dot{a}=\ddot{v}$ ) is not available. Any mode other than easy startup mode requires some form of real-time control, which is a challenge in itself and is tackled in section D.2.3 

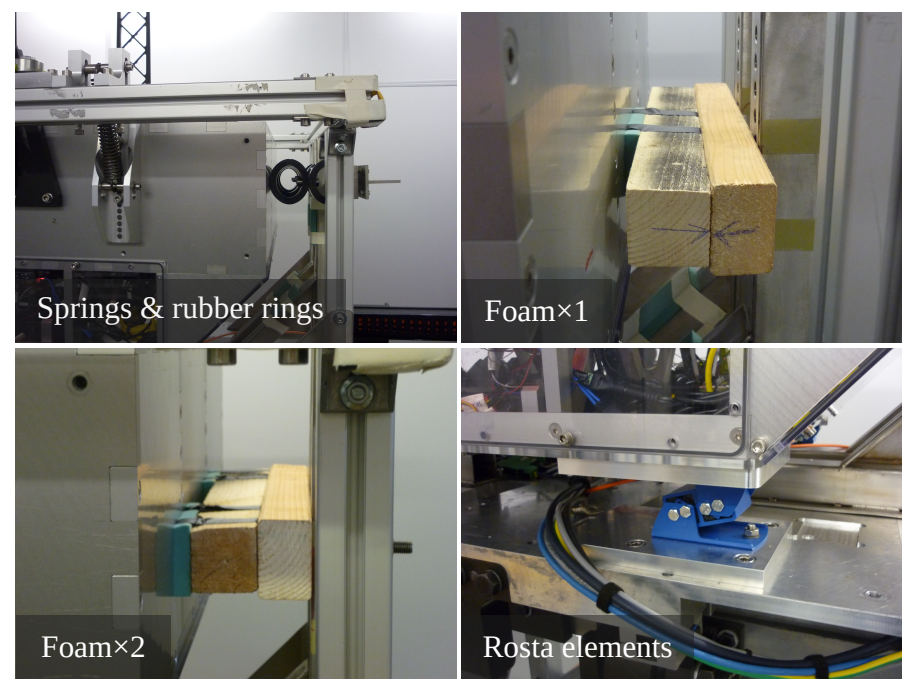

Figure D.1: The different suspensions and buffers that are considered in this section. There is a fifth combination not pictured here, which is using the Rosta elements and foam $\times 1$ simultaneously.

The rms acceleration is measured for 6 different velocities, as well as for standstill, both with the motor turned on and turned off. The latter allows for estimating the measurement noise. The rms accelerations are shown in table D.1. As can be seen already for $0.5 \mathrm{~m} \mathrm{~s}^{-1}$, the rms acceleration is $0.5 \mathrm{~m} \mathrm{~s}^{-2}$, which is equal to the Kolmogorov acceleration for a moderate dissipation rate of $0.01 \mathrm{~m}^{2} \mathrm{~s}^{-3}$. Hence, in order to measure droplet accelerations while the seesaw is running, the vibrations must be damped.

The frequency of the vibrations $f=v \cdot 80 \mathrm{~m}^{-1}$. The proportionality constant $80 \mathrm{~m}^{-1}$ equals $(1.25 \mathrm{~cm})^{-1}$, with $1.25 \mathrm{~cm}$ equal to $1 / 6$ of the motor's pole-pair distance.

\section{D.2.2 Passive damping}

The first approach taken is to passively damp the vibrations. To do so, the camera box is made part of a forced mass-spring system, where the camera box takes on the role of the mass, and the performance of various different springs is evaluated. Each spring consists of an element that suspends the box, i.e. that counteracts the box's weight, and an element that buffers the table's 
horizontal acceleration. The tested spring $\oint^{1}$ are: 1) springs and rubber rings; 2) springs and foam $\times 2$; 3) springs and foam $\times 1$; 4) Rosta ESL 18 elements; and 5) Rosta ESL 18 elements and foam $\times 1$. An overview of these is shown in figure D.1.

\section{Method}

Before evaluating a spring, the height of the suspension is adjusted so that the buffers are level with the box's centre of mass. That way no torque is generated, and the box won't start to rotate during the acceleration phase. To do this, the box is fitted with an inertial measurement unit (IMU, InvenSense MPU-6050), the gyroscope of which is used to measure the box's rotation rate. The motor is then commanded to move forth and back along the rails once, and depending on the measured rotation rate, the suspension's height is adjusted. This procedure is repeated until the resulting maximum rotation rate is sufficiently low.

Then, to evaluate a spring, the motor is again commanded to move forth and back, but now with the SDI accelerometers as well as the high-speed cameras active. The data from the accelerometers is used to analyse the camera box vibration. Reference measurements with the motor switched off are done as well.

All experiments discussed here, are performed with the high-speed cameras, optics, and other components installed in the camera box, so that its mass and mass distribution are representative of normal conditions.

\section{Analysis}

It is possible to model the box and spring as a mass-spring system, the parameters of which can be measured, and can be used to simulate how it would respond to certain acceleration profiles etc. However, here a more pragmatic approach is taken: the only two parameters of interest are

- $\Delta t_{d}$, the time needed for the box's vibrations due to acceleration or deceleration, to decay to below a certain threshold; and

- $a_{i, \mathrm{rms}}$, the rms of the box' vibrations during the constant velocity phase, measured after the acceleration vibrations have decayed.

Here the threshold for vibrations to decay to is chosen to be $\left|a_{x}\right|<0.1 \mathrm{~m} \mathrm{~s}^{-2}$, with $a_{x}$ the box's acceleration in the direction of travel. This threshold is

\footnotetext{
${ }^{1}$ Throughout this section "spring" refers to a combination of suspension and buffers.
} 

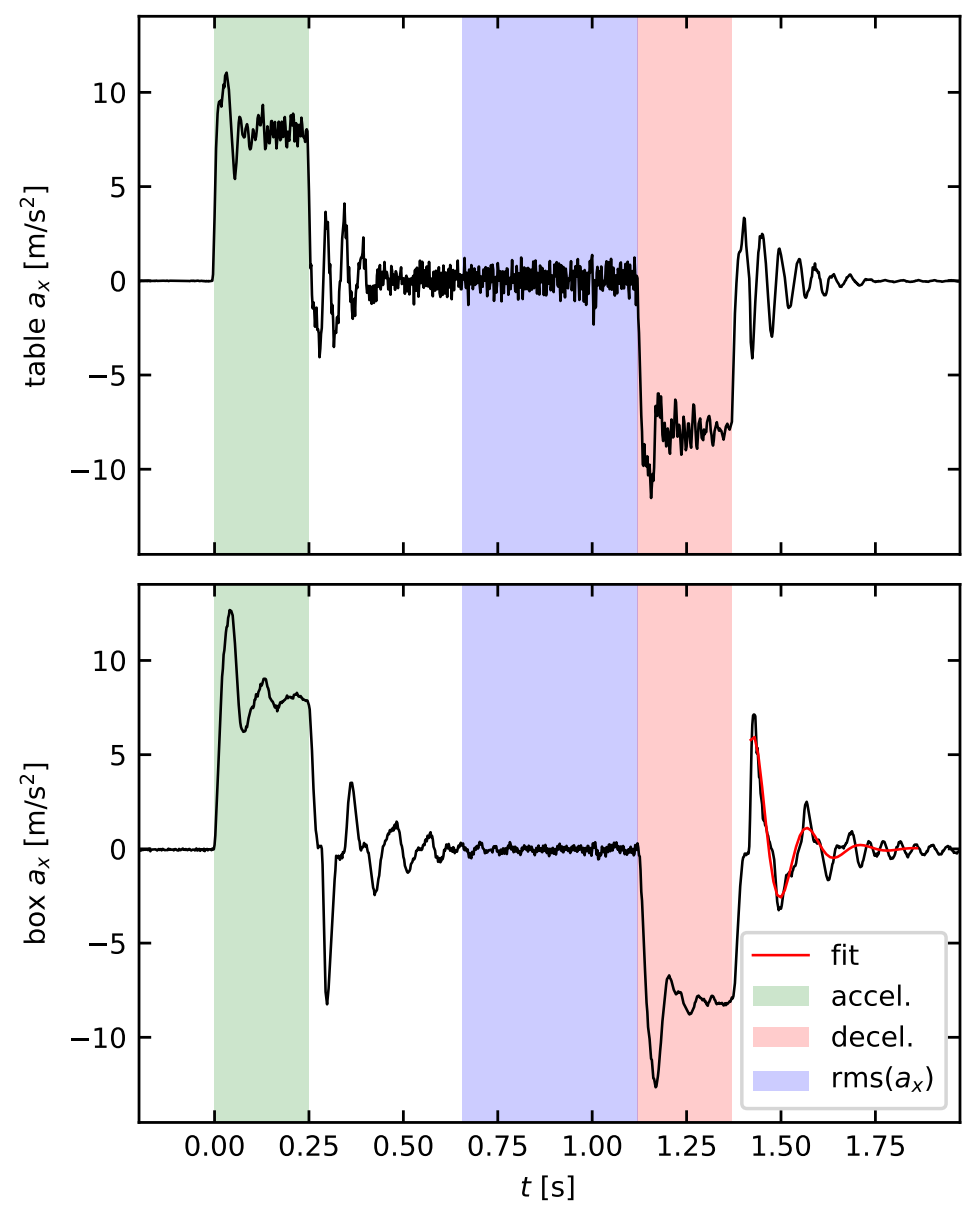

Figure D.2: Example of the analysis of the box's acceleration $a_{x}$ for a single experiment. The green region is the acceleration phase, the red region is the deceleration phase, and the blue region indicates where $a_{r m s}$ is computed. In this experiment the box was buffered using foam $\times 2, v=2.0 \mathrm{~m} \mathrm{~s}^{-1}, a=8.0 \mathrm{~m} \mathrm{~s}^{-2}$, and the resulting $\Delta t_{d}=0.40 \mathrm{~s}$ and $a_{r m s}=0.18 \mathrm{~m} \mathrm{~s}^{-2}$. 
approximately equal to $0.1 a_{\eta}$, with $a_{\eta}$ the Kolmogorov acceleration, a measure for the typical particle acceleration. The acceleration data is analysed as follows:

1. Load $a_{i}(t)$ (with $\left.i=x, y, z\right)$ and subtract the acceleration zero reference.

2. Find the acceleration phase's start time $t_{0}$; this is the earliest time for which $a_{x} \geq 0.2$.

3. Given the experiment's programmed velocity and acceleration, compute the acceleration phase's end time $t_{1}$, and the deceleration phase's start and end times $t_{2}$ and $t_{3}$.

4. Assuming that the vibrations decay after the deceleration phase as they do after the acceleration phase, it is easiest to fit them after the deceleration phase, because the motor isn't moving and doesn't introduce cogging torque. The fit equation is

$$
a_{x}=a_{0} \exp \left[-\left(t-t_{3}\right) / \tau\right] \cos \left(\omega t+\phi_{0}\right) .
$$

The time is offset by the deceleration phase's end time $t_{3}$.

5. Find $\Delta t_{d}$ : this is given by

$$
a_{0} \exp \left[-\Delta t_{d} / \tau\right]=0.1 \mathrm{~m} \mathrm{~s}^{-2} .
$$

6. If $t_{1}+\Delta t_{d}<t_{2}$, then compute $a_{i, \mathrm{rms}}=\operatorname{rms}\left(a_{i}\right)$ between these two points in time.

An example of this analysis is shown in figure D.2 Only the $x$-component of the acceleration is shown.

\section{Results}

Results of the above analysis are shown in table D.2. The best combination of suspension and buffers seems to be springs and foam $\times 1$. This combination has a low $\Delta t_{d}$, which makes the period during which LPT measurements can be done the longest. Furthermore it has low $a_{i, \text { rms }}$, always below $0.2 \mathrm{~m} \mathrm{~s}^{-2}$ and often also below $0.1 \mathrm{~m} \mathrm{~s}^{-2}$, so that absolute acceleration measurements can be done. A good second candidate is foam $\times 2$, which has smaller $\Delta t_{d}$, and smaller rms accelerations in the $y$ and $z$-direction, but larger rms acceleration in the $x$-direction. It can be seen that in general $a_{x, \text { rms }}<a_{y, \text { rms }}$ and $a_{x, \text { rms }}<a_{z, \text { rms }}$. 
Table D.2: Results of the vibration analysis for various combinations of suspension and buffers, and at various translation speeds $v$.

\begin{tabular}{|c|c|c|c|c|c|c|}
\hline \multirow{2}{*}{ Suspension } & \multirow{2}{*}{ Buffers } & \multirow{2}{*}{$v\left[\mathrm{~m} \mathrm{~s}^{-1}\right]$} & \multirow{2}{*}{$\Delta t_{d}[\mathrm{~s}]$} & \multicolumn{3}{|c|}{$a_{i, \mathrm{rms}}\left[\mathrm{m} \mathrm{s}^{-2}\right]$} \\
\hline & & & & $x$ & $y$ & $z$ \\
\hline \multirow{2}{*}{ springs } & \multirow{2}{*}{ rubber rings } & 1.0 & 3.44 & & - & \\
\hline & & 2.0 & 5.05 & & - & \\
\hline \multirow{3}{*}{ springs } & \multirow{3}{*}{ foam $\times 2$} & 0.5 & 0.48 & 0.57 & 0.03 & 0.05 \\
\hline & & 1.0 & 0.43 & 0.12 & 0.05 & 0.08 \\
\hline & & 2.0 & 0.40 & 0.18 & 0.07 & 0.12 \\
\hline \multirow{3}{*}{ springs } & \multirow{3}{*}{ foam $\times 1$} & 0.5 & 0.65 & 0.16 & 0.07 & 0.09 \\
\hline & & 1.0 & 0.59 & 0.06 & 0.07 & 0.09 \\
\hline & & 2.0 & 0.59 & 0.11 & 0.08 & 0.11 \\
\hline \multirow{3}{*}{ Rosta el. } & \multirow{3}{*}{ - } & 0.5 & 1.98 & 0.18 & 0.02 & 0.09 \\
\hline & & 1.0 & 2.01 & 0.22 & 0.04 & 0.03 \\
\hline & & 2.0 & 1.47 & & - & \\
\hline \multirow{3}{*}{ Rosta el. } & \multirow{3}{*}{ foam $\times 1$} & 0.5 & 0.42 & 0.14 & 0.02 & 0.16 \\
\hline & & 1.0 & 0.50 & 0.24 & 0.03 & 0.06 \\
\hline & & 2.0 & 0.47 & 0.17 & 0.08 & 0.12 \\
\hline
\end{tabular}




\section{D.2.3 Active damping}

The second approach is an actively damped approach. The box is still part of a mass-spring system, and a spring still consists of suspension of buffers, but the buffers now have variable, controllable stiffness. The stiffness is set high during acceleration and deceleration, so that the box closely follows the table's motion. During the constant velocity phase the stiffness is set low, so that the motor's cogging torque isn't transferred into the box.

The suspension used here are the springs that were also used for the passive damping approach; see figure D.1 The buffers are replaced by foam-padded pistons, that are actuated using solenoids. Three pistons and solenoids are mounted on either side of the box. One such side is shown on the next page. The height of each trio of pistons is adjusted, so that they clamp onto the box at the height of its centre of mass, and no torque is generated. Furthermore, the length of each piston is adjusted, such that when all pistons are actuated, the box isn't moved or rotated away from its resting position.

The solenoids are of type GTCA070X20A02 (Magnet-Schultz GmbH, Memmingen, Germany). Each solenoid is coupled to a piston through a 2:1 lever. The maximum force exerted by a set of three pistons ranges from $516 \mathrm{~N}$ to $1458 \mathrm{~N}$, depending on the position in the solenoids' strokes. The maximum acceleration is set by the force the pistons can hold and the weight of the camera box. The pistons are spring-loaded, and move away from the box when they are not actuated. A side view of a piston and solenoid is shown in the inset on the next page.

The solenoids are controlled using a programme that runs on the IndraDrive's programmable logic controller (PLC). It actuates the solenoids slightly before the acceleration/deceleration phases start, and releases them at the end of these phases.

The PLC is furthermore used to impose a jerk-limited motion profile on the table. By using such a profile, the box will hopefully have no motion w.r.t. to the table, and will be at its resting position, at the beginning of the constant velocity phase. This way the box should not start swinging when the pistons are released. The chosen acceleration profile is an inverted parabola, so that acceleration is zero at the beginning and end of the acceleration phase.

For the purposes of these tests the PLC is given full control over the motor. The PLC, in turn, is controlled by setting the PLC registers through the BoschRexroth software.

A number of experiments are conducted to see how well the system performs. The maximum acceleration is set to either 4,6 , or $8 \mathrm{~m} \mathrm{~s}^{-2}$, and the velocity is set to either $1.0,1.5,2.0$, or $2.5 \mathrm{~m} \mathrm{~s}^{-1}$. The solenoids are actuated 


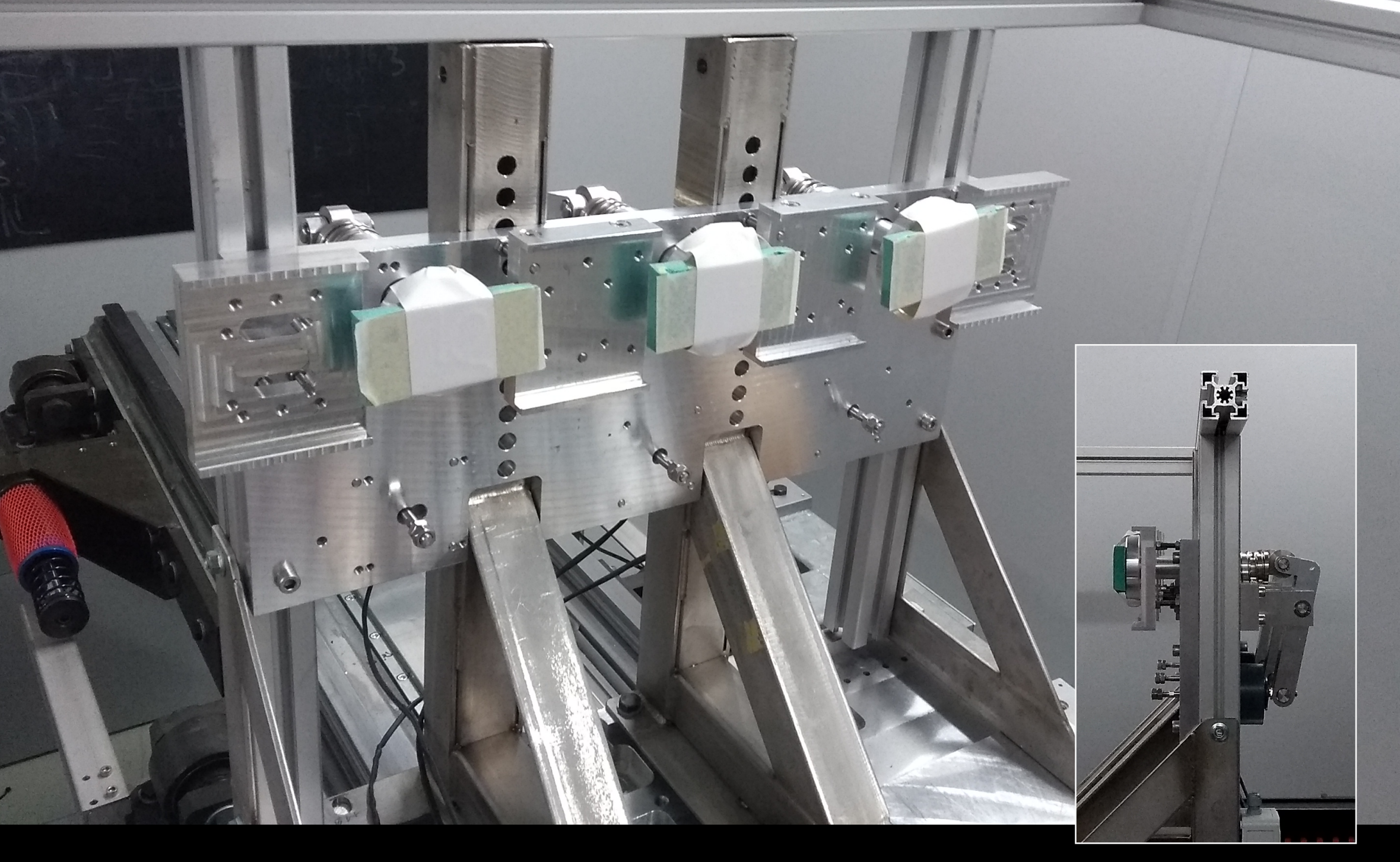


Table D.3: Results of the vibration analysis for various combinations of table velocity $v$ and acceleration $a$. The vibrations are quantified by means of the RMS acceleration of the camera box, measured during the period in which the box is free, i.e. when the pistons are not touching the box. The length of the free period $T_{\text {free }}$ is shown as well. For some combinations of velocity and acceleration there was no such period, which is indicated by dashes.

\begin{tabular}{cccccc}
\hline $\begin{array}{c}\text { Velocity } \\
{\left[\mathrm{m} \mathrm{s}^{-1}\right]}\end{array}$ & $\begin{array}{c}\text { Max. accel. } \\
{\left[\mathrm{m} \mathrm{s}^{-2}\right]}\end{array}$ & $\begin{array}{c}T_{\text {free }} \\
{[\mathrm{s}]}\end{array}$ & \multicolumn{3}{c}{ RMS acceleration $\left[\mathrm{m} \mathrm{s}^{-2}\right]$} \\
\hline 1.0 & 4.0 & 1.14 & 0.07 & 0.04 & $z$ \\
1.0 & 6.0 & 1.26 & 0.07 & 0.04 & 0.33 \\
1.0 & 8.0 & 1.32 & 0.09 & 0.05 & 0.35 \\
1.5 & 4.0 & 0.12 & 0.10 & 0.05 & 0.55 \\
1.5 & 6.0 & 0.31 & 0.10 & 0.06 & 0.46 \\
1.5 & 8.0 & 0.40 & 0.13 & 0.06 & 0.48 \\
2.0 & 4.0 & - & - & - & - \\
2.0 & 6.0 & - & - & - & - \\
2.0 & 8.0 & - & - & - & - \\
2.5 & 8.0 & - & - & - & - \\
\hline
\end{tabular}

$0.3 \mathrm{~s}$ before acceleration starts, and again $0.2 \mathrm{~s}$ before deceleration starts. The SDI accelerometers are again used to measure the table's and box's acceleration. An example of the measured acceleration is shown in figure D.3. Based on these experiments, the following observations can be made:

- In the current setup, the pistons cannot be moved far enough down, to the height of the box's centre of mass. As a result they exert torque on the box during the acceleration phase, and the box starts rotating.

- The pistons release the box too slowly. According to the datasheet, the solenoids have a release time of $95 \mathrm{~ms}$, however in practice it seems to take at least $0.7 \mathrm{~s}$ for the pistons to move away from the box.

It is currently not known whether only the pistons, or both the pistons and the solenoids move too slowly. If the solenoids are too slow, this could possibly be remedied by using a solenoid driver instead of a relay. Such a driver could help the magnetic flux in the solenoids to build up and decay quicker.

Despite the above observations, it is possible to get some figures on the performance of the active damping. To do so, the box's rms accelerations are 

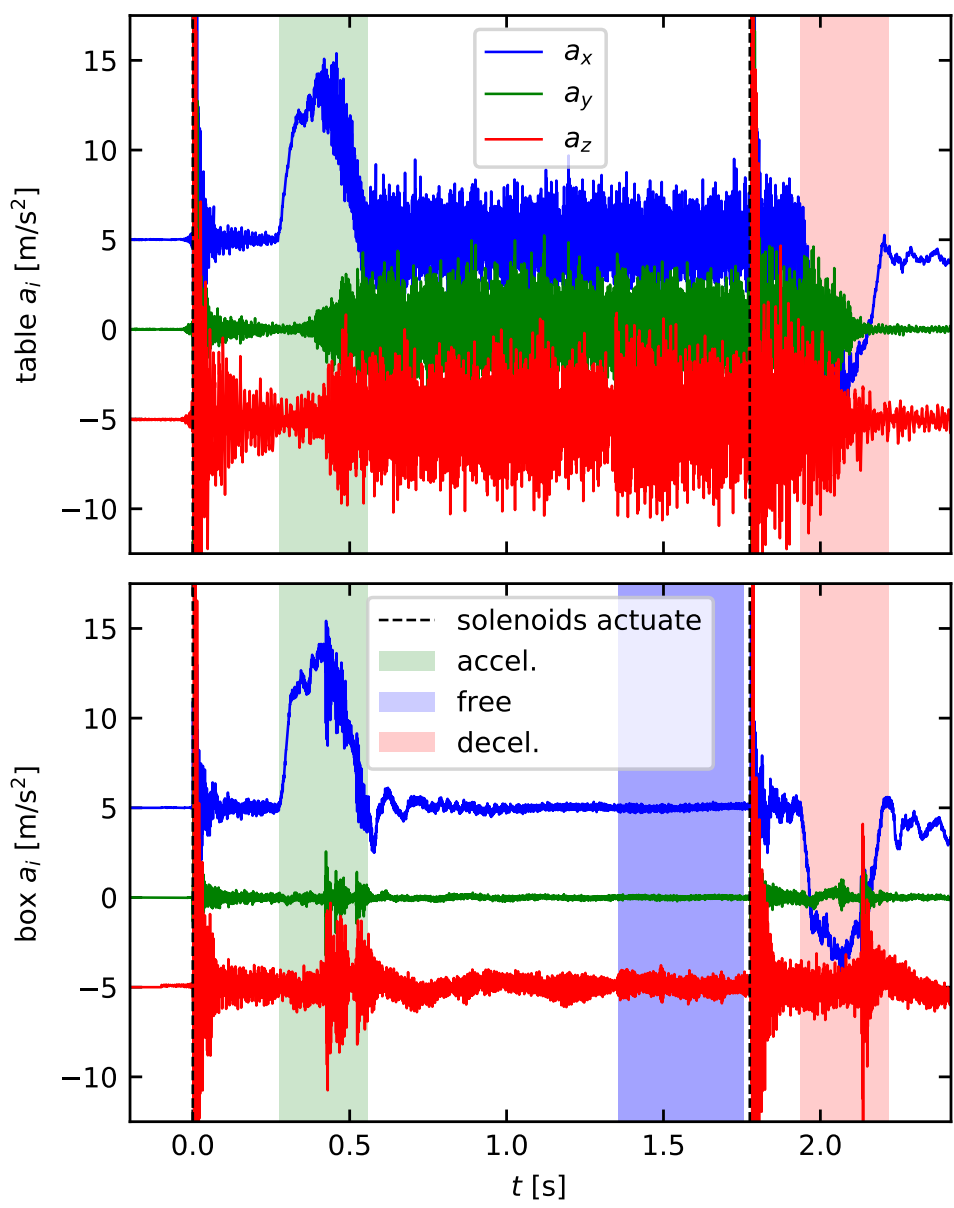

Figure D.3: Example of the box's accelerations $a_{i}$ for a single experiment. The green region is the acceleration phase, the red region is the deceleration phase. The blue region is the free period, during which the pistons should not be touching the box. The dashed lines indicate when the solenoids were actuated. The solenoids were released again at the end of the acceleration/deceleration phases. In this experiment $v=1.5 \mathrm{~m} \mathrm{~s}^{-1}$, and $a_{\max }=8.0 \mathrm{~m} \mathrm{~s}^{-2}$. 
computed between $0.8 \mathrm{~s}$ after the start of the constant velocity phase, and $0.02 \mathrm{~s}$ before the solenoids activate for the deceleration phase. This is the free period, during which the box should be undisturbed by the pistons; it is shaded blue in figure D.3. The results are shown in table D.3.

It is noteworthy that the rms acceleration in the z-direction is large compared to the other directions, too large to measure absolute droplet accelerations. This was generally not the case with the passive damping, and the cause of it is not known.

These results presented in table D.3 must be interpreted with caution, because of the aforementioned caveats, and because the behaviour of the active-damping system is not sufficiently understood yet. In particular the large rms accelerations in the $z$-direction should not be taken as a reason to dismiss the active damping system; further investigation is needed to improve its performance.

Finally, it should be mentioned that acceleration measurements from the camera box can be fed back to the motor's PLC, which can then correct the table's position to minimise the vibrations in real-time. The development of such a system would require a large amount of technical expertise, however.

\section{D.3 Conclusion}

The passive damping system is very promising, however it has not been tested in the field yet. It should be possible to take the current implementation and deploy it at the Schneefernerhaus as-is.

The active damping system is promising as well, but another iteration in the laboratory should be done, before it is taken to the Schneefernerhaus. By employing an active feedback system, it might be able to dampen the vibrations more effectively than the passive damping system can.

The use of the mean wind compensation comes with a large caveat: the time during which the camera box has a constant velocity can be rather short, in particular for high mean wind speeds. This restricts the amount of time during which useful particle tracking data can be acquired.

Finally, it should be noted that the development of both systems took a lot of effort, and is not finished yet. Whether the benefits of further development outweigh the drawbacks of the distraction that it poses, should require careful consideration, and should depend on the necessity of having mean wind compensation for the scientific question at hand. 


\section{Appendix E}

\section{Graphs}

\section{E.1 Turbulent statistics}

The shaded areas in these graphs indicates when the particle tracking experiment was running. 
25-9-2019
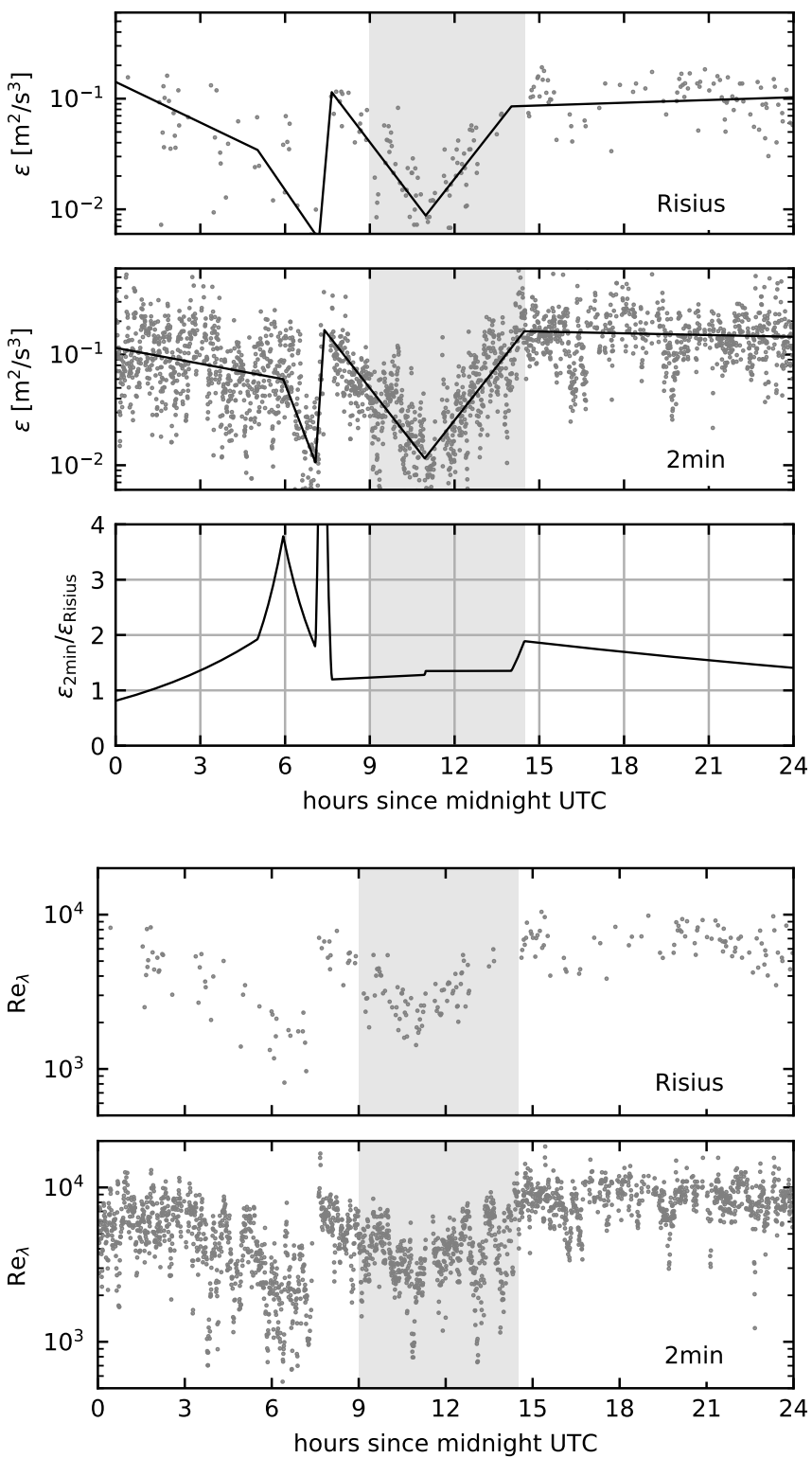
26-9-2019
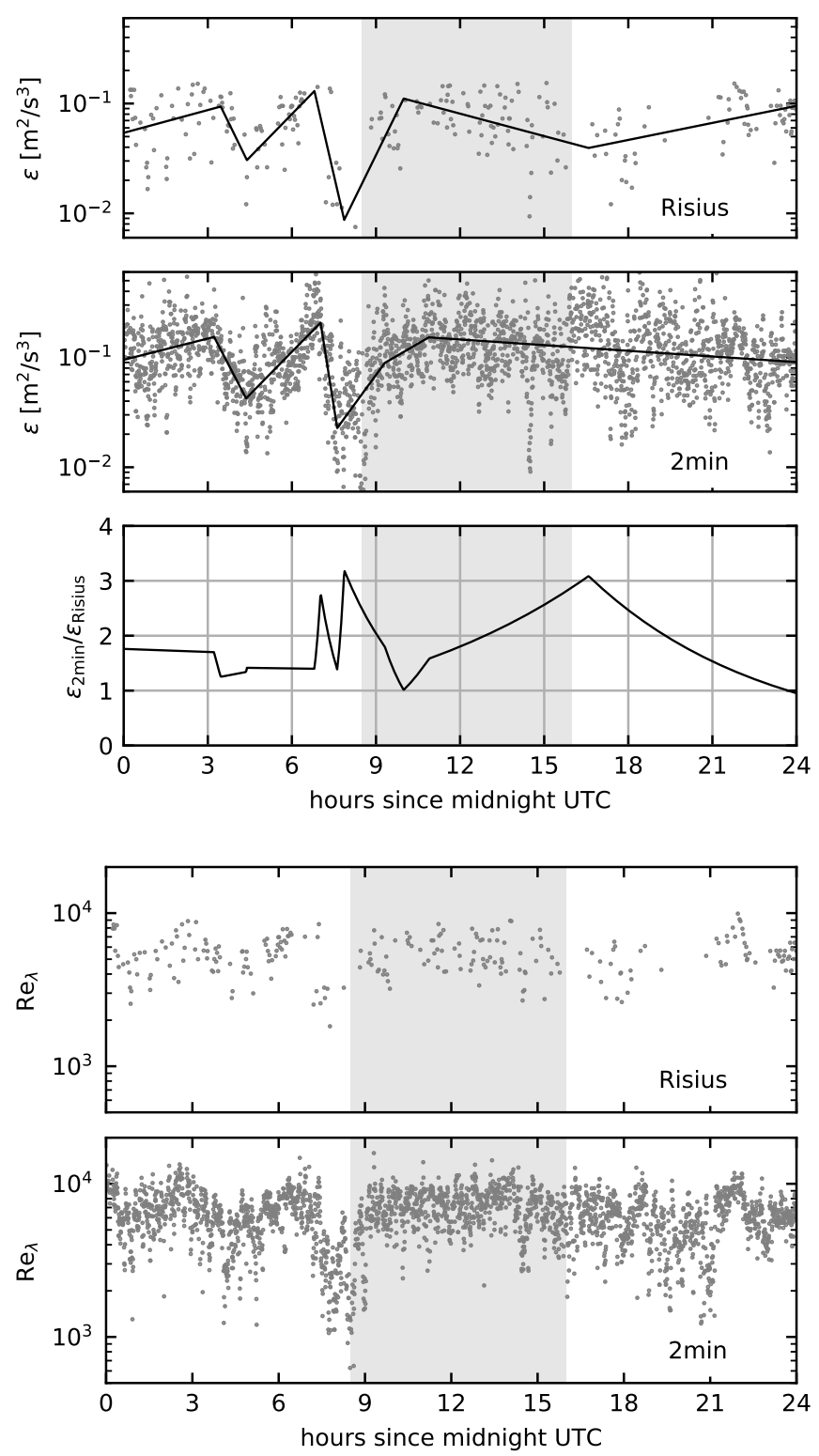
28-9-2019
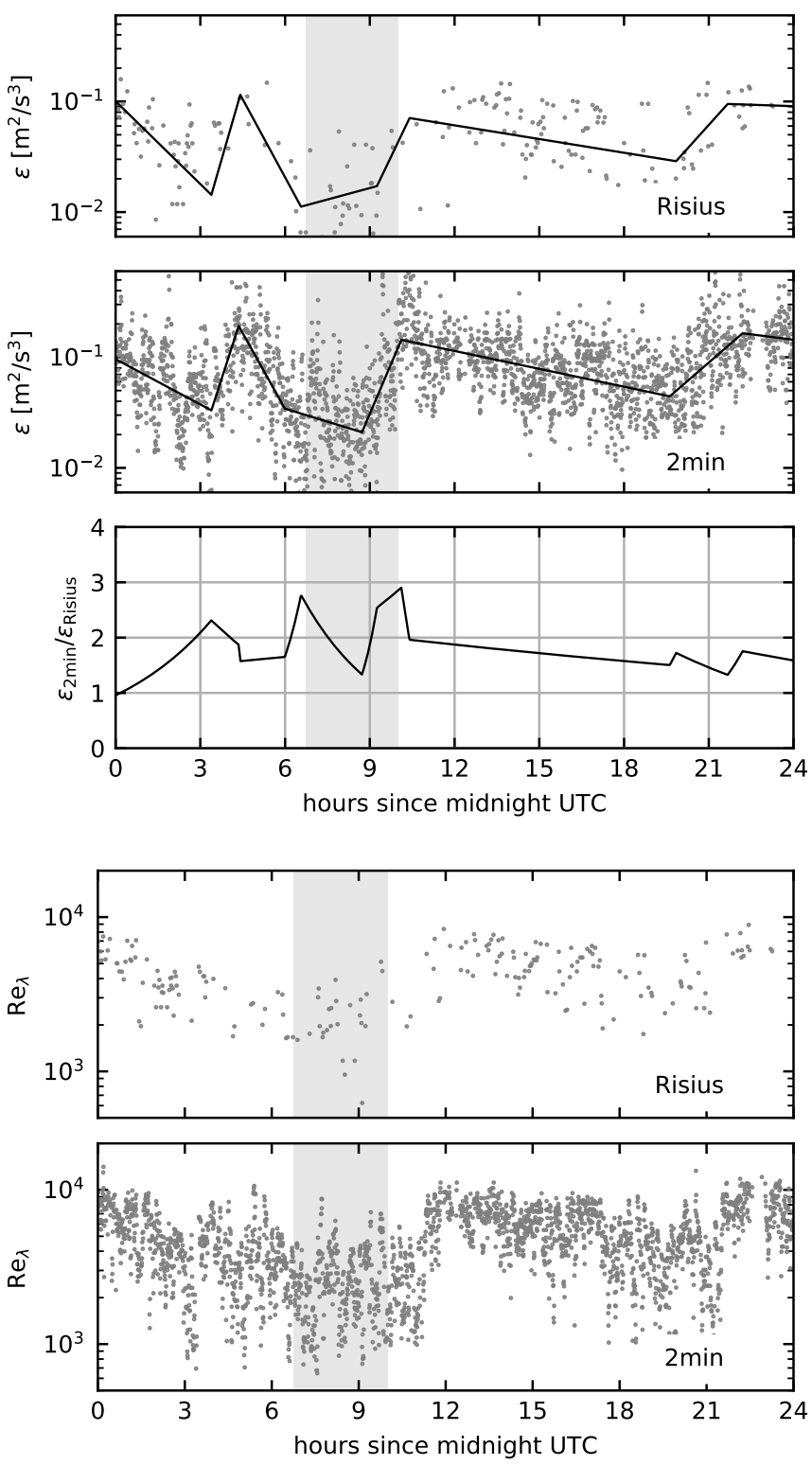


\section{E.2 Droplet accelerations}
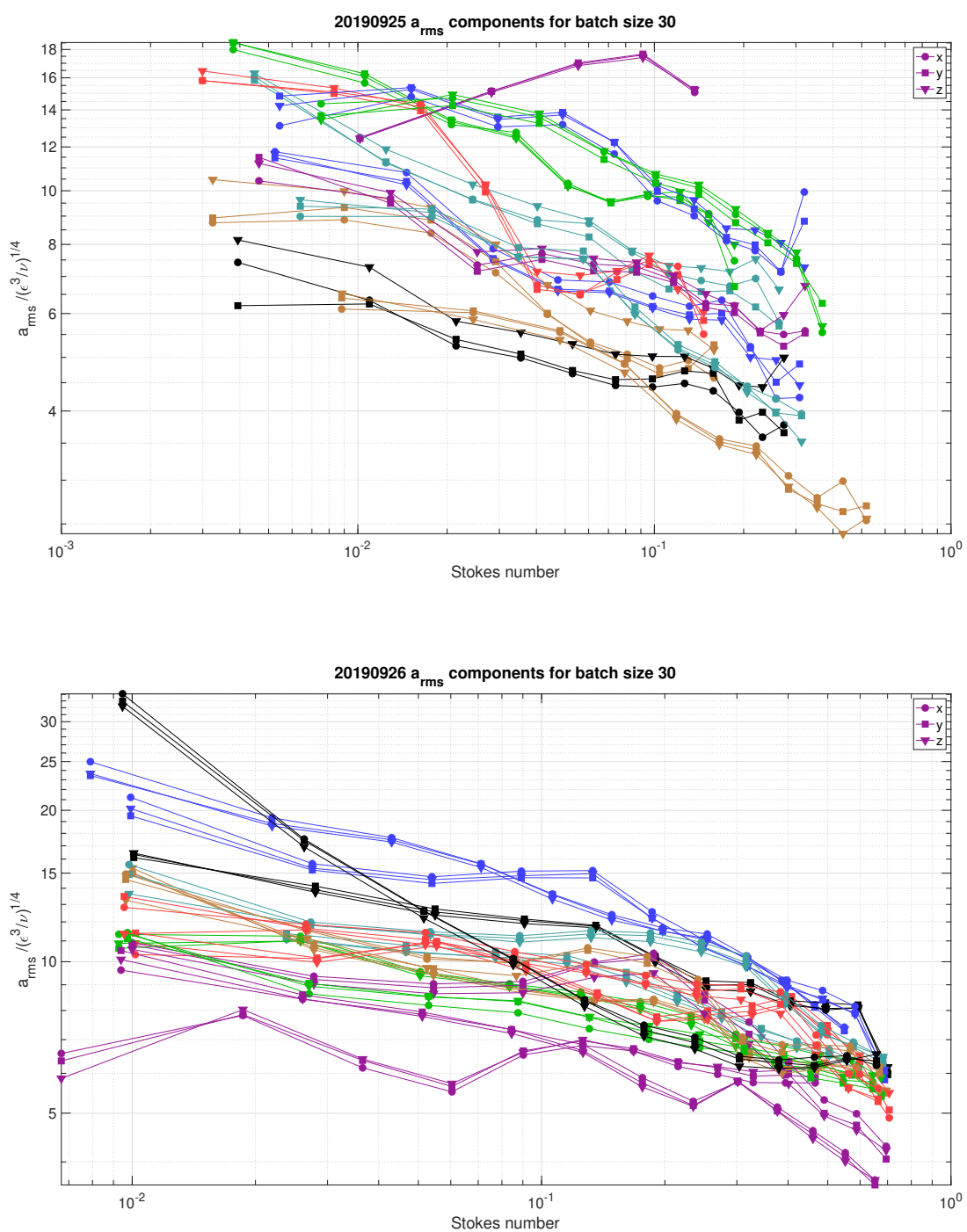


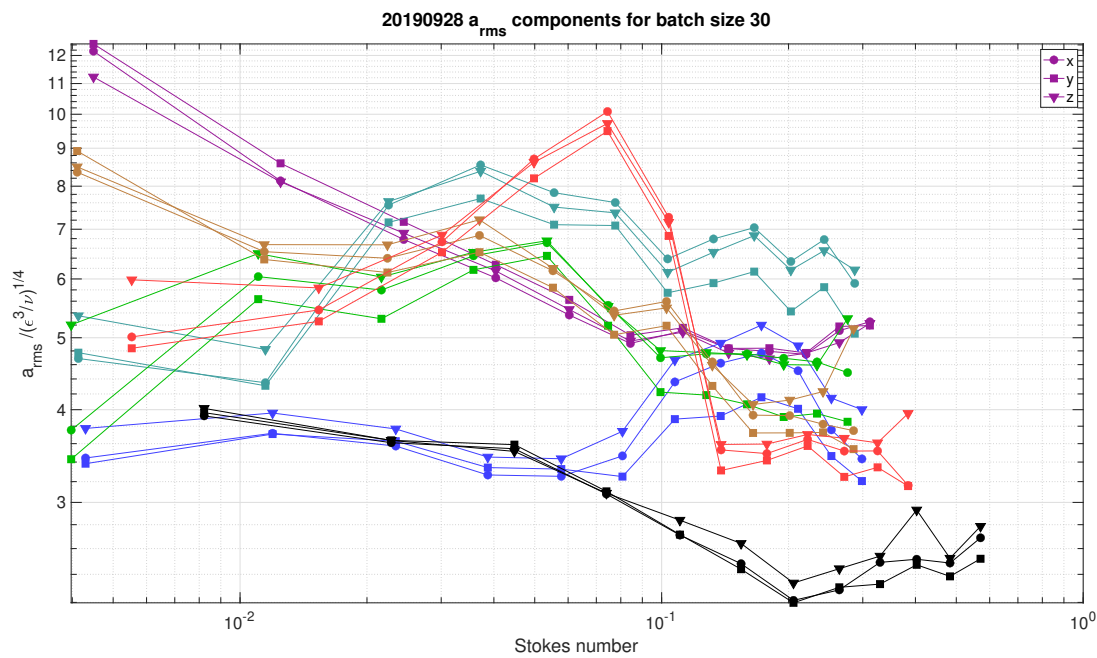

\section{E.3 Droplet acceleration fits}

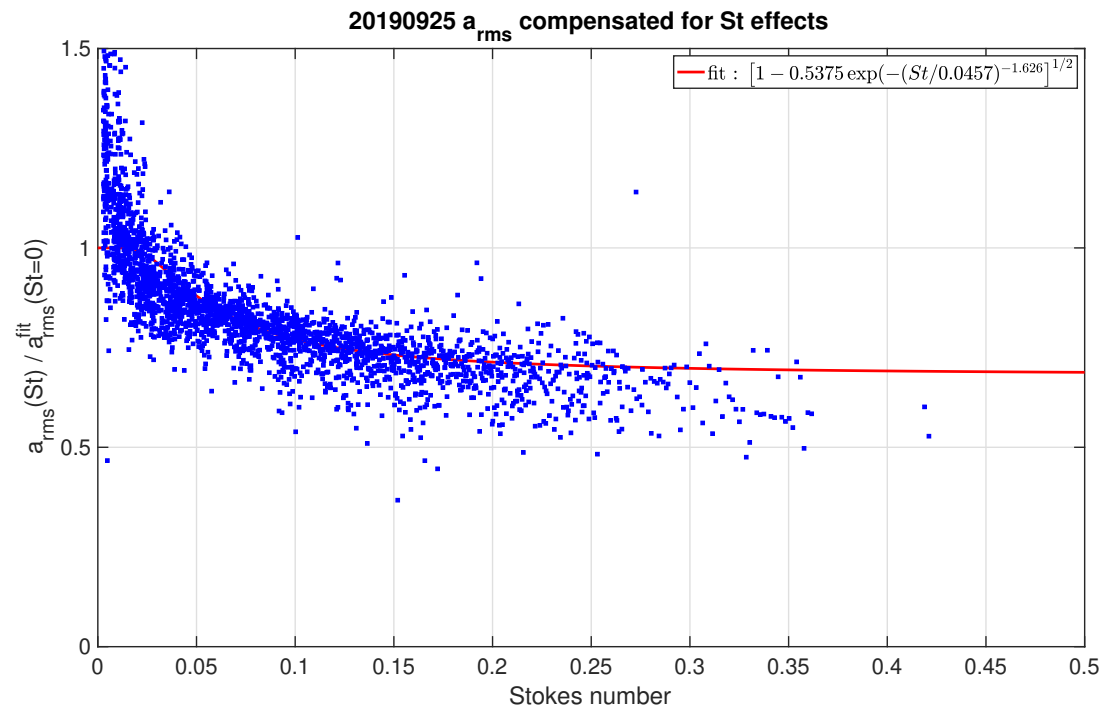



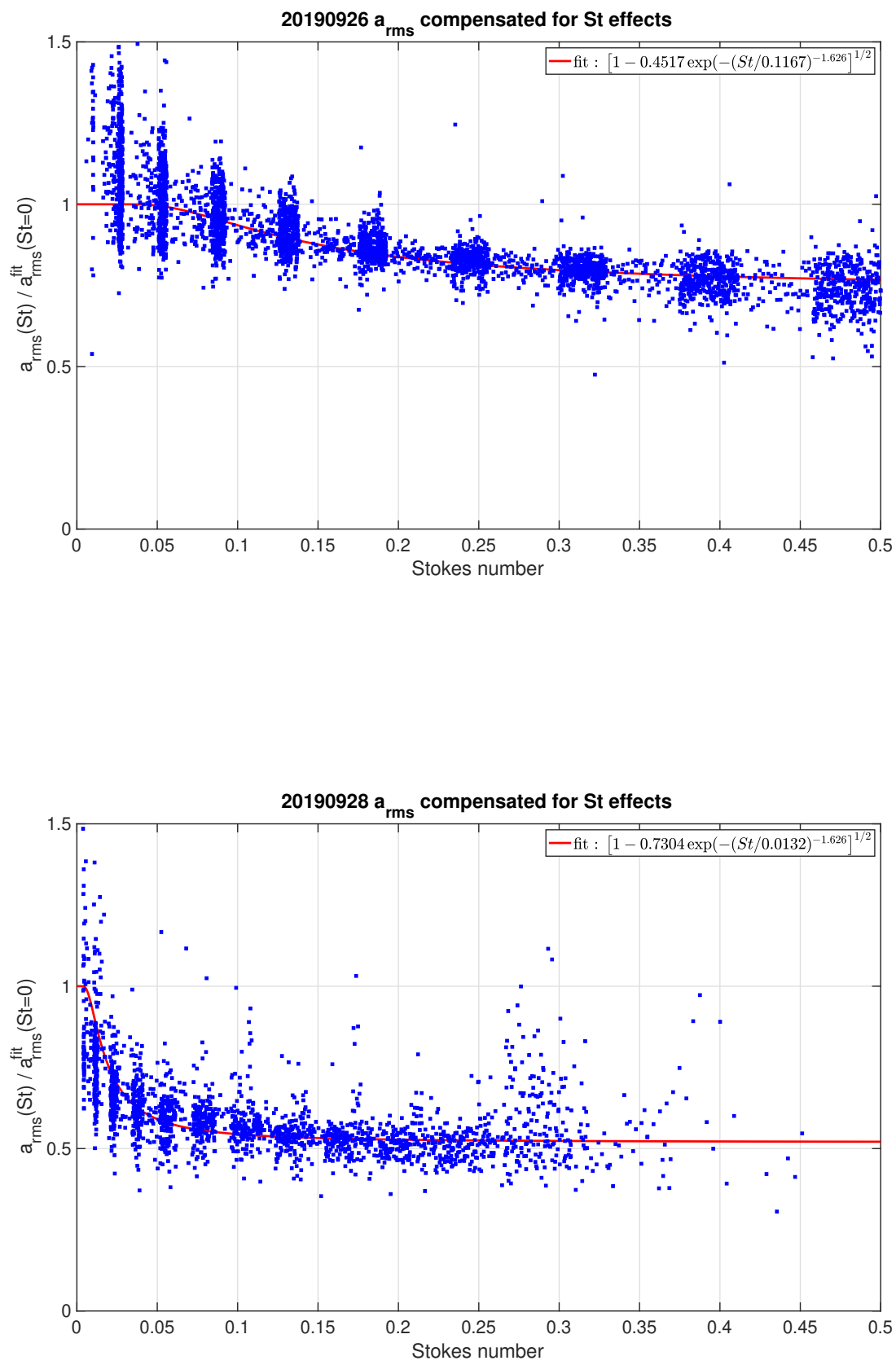


\section{E.4 Clustering exponent fits}
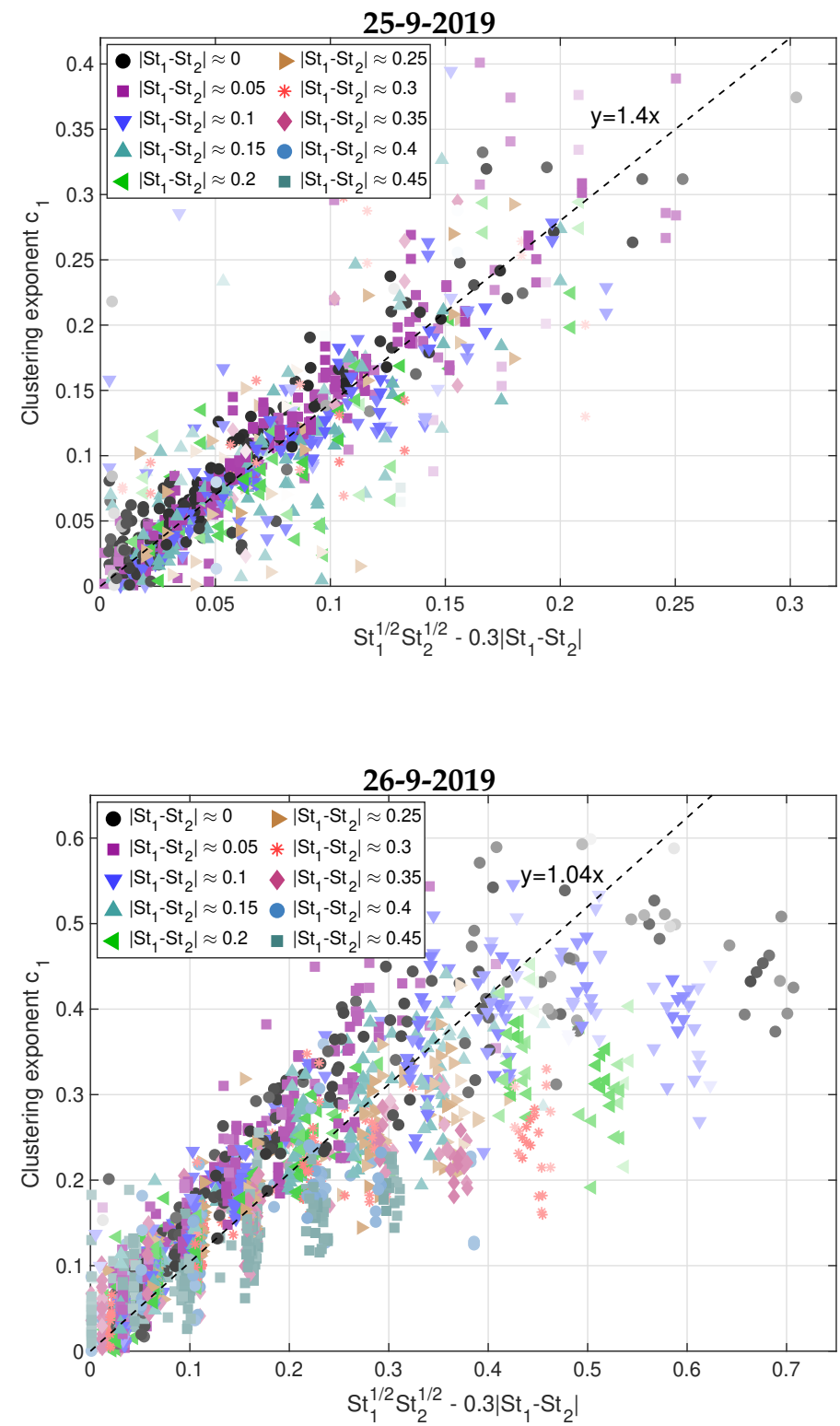


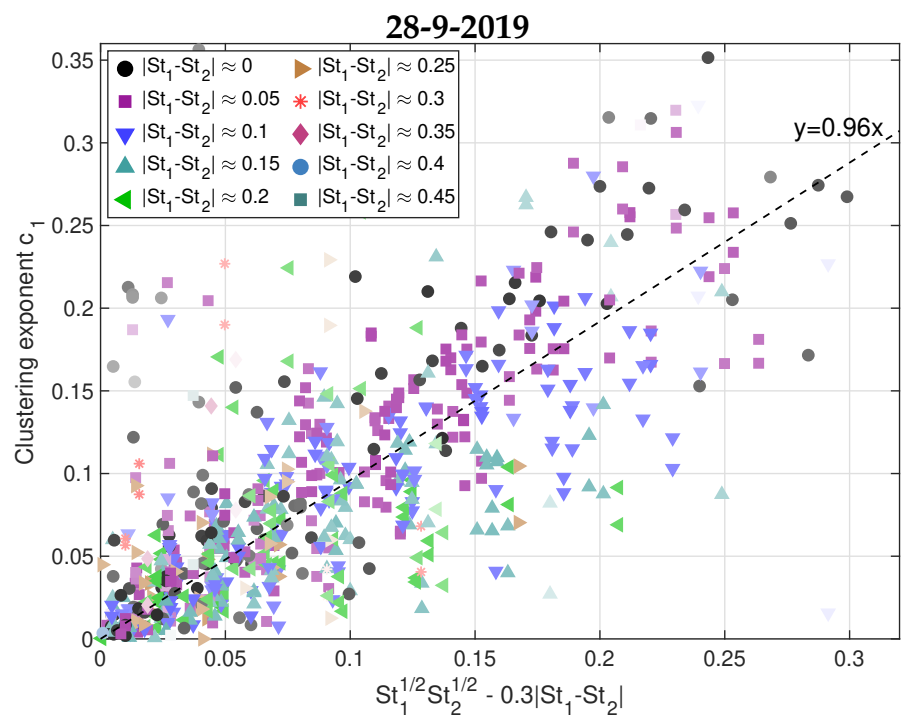




\section{Bibliography}

[1] V. M. Alipchenkov, L. I. Zaichik, and O. F. Petrov. High Temperature, 42 (6):919-927, November 2004. doi: 10.1007/s10740-005-0037-0.

[2] Orlando Ayala, Bogdan Rosa, and Lian-Ping Wang. New Journal of Physics, 10(7):075016, July 2008. doi: 10.1088/1367-2630/10/7/075016.

[3] Mohsen Bagheri and Eberhard Bodenschatz. URL http://eurec4a.eu/ platforms/cloudkite.

[4] J. Bec, L. Biferale, G. Boffetta, M. Cencini, S. Musacchio, and F. Toschi. Phys. Fluids, 18(9), 2006. doi: 10.1063/1.2349587.

[5] J. Bec, L. Biferale, M. Cencini, A. Lanotte, S. Musacchio, and F. Toschi. Phys. Rev. Lett., 98(8):084502, 2007. doi: 10.1103/PhysRevLett.98.084502.

[6] G. Bertens. Droplets and turbulence. Master's thesis, Eindhoven University of Technology, March 2015.

[7] H. Bocanegra Evans, N. Dam, Dennis van der Voort, Guus Bertens, and Willem van de Water. Review of Scientific Instruments, 86(2):023709, February 2015. doi: 10.1063/1.4909537.

[8] E. Bodenschatz, G. P. Bewley, H. Nobach, M. Sinhuber, and H. Xu. Rev. Sci. Instrum., 85(9):093908, 2014. doi: 10.1063/1.4896138.

[9] Craig F. Bohren and Donald R. Huffman. Absorption and Scattering of Light by Small Particles. Wiley, apr 1998. doi: 10.1002/9783527618156.

[10] Craig F. Bohren, Donald R. Huffman, and Bruce T. Draine. URL https: //www.astro.princeton.edu/ draine/scattering.html alternative: http: //scatterlib.wikidot.com/mie 
[11] Hakki Ergun Cekli and Willem van de Water. Experiments in Fluids, 49 (2):409-416, January 2010. doi: 10.1007/s00348-009-0812-5.

[12] J. Chun, D. L. Koch, S. L. Rani, A. Ahluwalia, and L. R. Collins. Journal of Fluid Mechanics, 536:219-251, July 2005. doi: 10.1017/s0022112005004568.

[13] Nils Damaschke, Holger Nobach, Thomas I. Nonn, Nikolay Semidetnov, and Cameron Tropea. Experiments in Fluids, 39(2):336-350, June 2005. doi: $10.1007 /$ s00348-005-1009-1.

[14] B. J. Devenish, P. Bartello, J.-L. Brenguier, L. R. Collins, W. W. Grabowski, R. H. A. IJzermans, S. P. Malinowski, M. W. Reeks, J. C. Vassilicos, L.-P. Wang, and Z. Warhaft. Quarterly Journal of the Royal Meteorological Society, 138(667):1401-1429, February 2012. doi: 10.1002/qj.1897.

[15] Hongxu Duan, Francisco J. Romay, Cheng Li, Amir Naqwi, Weiwei Deng, and Benjamin Y. H. Liu. Aerosol Science and Technology, 50(1): 17-25, November 2015. doi: 10.1080/02786826.2015.1123213.

[16] G. Falkovich, A Fouxon, and M G Stepanov. Nature, 419:151-154, 2002. doi: $10.1038 /$ nature00983.

[17] A. J. Favre, J. J. Gaviglio, and R. J. Dumas. Journal of Fluid Mechanics, 3(4): 344-356, January 1958. doi: 10.1017/s0022112058000021.

[18] M. J. Fisher and P. O. A. L. Davies. Journal of Fluid Mechanics, 18(1):97-116, January 1964. doi: 10.1017/s0022112064000076.

[19] Piotr J. Flatau. URL http://scatterlib.wikidot.com/

[20] OPC Foundation. Opc unified architecture. URL https://opcfoundation. org/about/opc-technologies/opc-ua/.

[21] Itzhak Fouxon, Yongnam Park, Roei Harduf, and Changhoon Lee. Physical Review E, 92(3), September 2015. doi: 10.1103/physreve.92.033001.

[22] J. C. H. Fung, J. C. R. Hunt, N. A. Malik, and R. J. Perkins. Journal of Fluid Mechanics, 236:281-318, March 1992. doi: 10.1017/s0022112092001423.

[23] R. S. Gao, A. E. Perring, T. D. Thornberry, A. W. Rollins, J. P. Schwarz, S. J. Ciciora, and D. W. Fahey. Aerosol Science and Technology, 47(2):137-145, February 2013. doi: 10.1080/02786826.2012.733039. 
[24] Wojciech W. Grabowski and Lian-Ping Wang. Annual Review of Fluid Mechanics, 45(1):293-324, January 2013. doi: 10.1146/ annurev-fluid-011212-140750.

[25] K. Gustavsson and B. Mehlig. Advances in Physics, 65(1):1-57, January 2016. doi: 10.1080/00018732.2016.1164490.

[26] Trevor Hastie, Robert Tibshirani, and Jerome Friedman. The Elements of Statistical Learning. Springer New York, 2009. doi: 10.1007/ 978-0-387-84858-7. URL https://doi.org/10.1007/978-0-387-84858-7.

[27] R. Jason Hearst and Bharathram Ganapathisubramani. Wind Energy, 20 (12):2021-2035, August 2017. doi: 10.1002/we.2138.

[28] Michael Heim, Benjamin J. Mullins, Heinz Umhauer, and Gerhard Kasper. Journal of Aerosol Science, 39(12):1019-1031, December 2008. doi: 10.1016/j.jaerosci.2008.07.006.

[29] Reginald J. Hill. Journal of Fluid Mechanics, 452:361-370, February 2002. doi: 10.1017/s0022112001007091.

[30] R.J. Hill. Atmospheric Research, 40(2-4):153-175, May 1996. doi: 10.1016/ 0169-8095(95)00032-1.

[31] Don Holve and Sidney A. Self. Applied Optics, 18(10):1632, May 1979. doi: 10.1364/ao.18.001632.

[32] Alexei Kiselev, Heike Wex, Frank Stratmann, Alexandre Nadeev, and Dmitry Karpushenko. Applied Optics, 44(22):4693, August 2005. doi: 10.1364/ao.44.004693.

[33] A. N. Kolmogorov. Proc. Roy. Soc. London, 434(1890):9-13, 1991. doi: 10.1098/rspa.1991.0075.

[34] C. Küchler, G. Bewley, and E. Bodenschatz. J. Stat. Phys., 175(3):617-639, 2019. doi: $10.1007 /$ s10955-019-02251-1.

[35] S. Lance, C. A. Brock, D. Rogers, and J. A. Gordon. Atmospheric Measurement Techniques, 3(6):1683-1706, December 2010. doi: 10.5194/ amt-3-1683-2010.

[36] Mathieu Legrand, José Nogueira, Antonio Lecuona, and Ana Hernando. Experimental Thermal and Fluid Science, 76:135-145, September 2016. doi: 10.1016/j.expthermflusci.2016.03.018. 
[37] C. C. Lin. Quarterly of Applied Mathematics, 10(4):295-306, January 1953. doi: $10.1090 /$ qam/51649.

[38] Jiang Lu, Hansen Nordsiek, Ewe Wei Saw, and Raymond A. Shaw. Physical Review Letters, 104(18), May 2010. doi: 10.1103/physrevlett.104.184505.

[39] J. L. Lumley. Physics of Fluids, 8(6):1056, 1965. doi: 10.1063/1.1761355.

[40] David J. C. MacKay. Information Theory, Inference, and Learning Algorithms. Cambridge University Press, 4 edition, 2005.

[41] M. R. Maxey and J. J. Riley. Phys. Fluids, 26(4):883-889, 1983. doi: 10. 1063/1.864230.

[42] Franz Mayinger and Oliver Feldmann, editors. Optical Measurements. Springer Berlin Heidelberg, 2001. doi: 10.1007/978-3-642-56443-7.

[43] Geoffrey J. McLachlan and Thriyambakam Krishnan. The EM algorithm and extensions. Wiley series in probability and statistics. Wiley, 2 edition, 2008. ISBN 978-0-471-20170-0.

[44] Katia Moskvitch. Nature, August 2014. doi: 10.1038/nature.2014.15659.

[45] S. Murray, M. F. Lightstone, and S. Tullis. Physics of Fluids, 28(3):033302, March 2016. doi: 10.1063/1.4942815.

[46] S. Murray, M. F. Lightstone, and S. Tullis. Physical Review E, 94(3), September 2016. doi: 10.1103/physreve.94.033303.

[47] F. T. M. Nieuwstadt. Turbulentie. Epsilon Uitgaven, Utrecht, 2 edition, 1998. ISBN 9050410286.

[48] S. B. Pope. Turbulent Flows. Cambridge University Press, 2000. ISBN 9780521598866.

[49] H.R. Pruppacher and J.D. Klett. Microphysics of Clouds and Precipitation. Springer Netherlands, 2010. doi: 10.1007/978-0-306-48100-0.

[50] L. F. Richardson. Weather prediction by numerical process. 1922.

[51] S. Risius. Investigation of turbulent flows at the umweltforschungsstation schneefernerhaus (zugspitze). Master's thesis, Georg-August University Göttingen, 2012. URL http://www.lfpn.ds.mpg.de/turbulence/documents/ Diplom-Zugspitze-Steffen-Risius.pdf. 
[52] S. Risius, H. Xu, F. Di Lorenzo, H. Xi, H. Siebert, R. A. Shaw, and E. Bodenschatz. Atmospheric Measurement Techniques, 8(8):3209-3218, August 2015. doi: 10.5194/amt-8-3209-2015.

[53] Steffen Risius. Investigation of turbulent flows at the umweltforschungsstation schneefernerhaus (zugspitze). Master's thesis, GeorgAugust University Göttingen, March 2012.

[54] S. G. Saddoughi and S. V. Veeravalli. J. Fluid Mech., 268:333-372, 1994. doi: $10.1017 /$ S0022112094001370.

[55] P. G. Saffman and J. S. Turner. Journal of Fluid Mechanics, 1(01):16, May 1956. doi: $10.1017 / \mathrm{s} 0022112056000020$.

[56] J. P. L. C. Salazar and L. R. Collins. Annu. Rev. Fluid Mech., 41(1):405-432, 2009. doi: 10.1146/annurev.fluid.40.111406.102224.

[57] J. P. L. C. Salazar, J. de Jong, L. Cao, SS. H. Woodward, H. Meng, and L. R. Collins. Journal of Fluid Mechanics, 600:245-256, March 2008. doi: $10.1017 / \mathrm{s} 0022112008000372$.

[58] D. Schanz, S. Gesemann, and A. Schröder. Exp. Fluids, 57(5):1-27, 2016. doi: 10.1007/s00348-016-2157-1. Article 70, Published online: 27 April 2016.

[59] Oliver Schlenczek. Airborne and ground-based holographic measurement of hydrometeors in liquid-phase, mixed-phase and ice clouds. PhD thesis, 2018. URL https://openscience.ub.uni-mainz.de/handle/20.500.12030/4126.

[60] R. A. Shaw. Annu. Rev. Fluid Mech., 35(1):183-227, 2003. doi: 10.1146/ annurev.fluid.35.101101.161125.

[61] H. Siebert, R. A. Shaw, J. Ditas, T. Schmeissner, S. P. Malinowski, E. Bodenschatz, and H. Xu. Atmospheric Measurement Techniques, 8(8):3219-3228, August 2015. doi: 10.5194/amt-8-3219-2015.

[62] Bjorn Stevens, Sandrine Bony, David Farrell, Felix Ament, Alan M. Blyth, Christopher W. Fairall, Johannes Karstensen, Patricia K. Quinn, Sabrina Speich, Claudia Acquistapace, Franziska Aemisegger, Anna Lea Albright, Hugo Bellenger, Eberhard Bodenschatz, Kathy-Ann Caesar, Rebecca Chewitt-Lucas, Gijs de Boer, Julien Delanoë, Leif Christopher Denby, Florian Ewald, Benjamin Fildier, Marvin Forde, Geet George, Silke Gross, Martin Hagen, Andrea Hausold, Karen J. Heywood, Lutz 
Hirsch, Marek Jacob, Friedhelm Jansen, Stefan Kinne, Daniel Klocke, Tobias Kölling, Heike Konow, Marie Lothon, Wiebke Mohr, Ann Kristin Naumann, Louise Nuijens, Léa Olivier, Robert Pincus, Mira L. Pöhlker, Gilles Reverdin, Gregory Roberts, Sabrina Schnitt, Hauke Schulz, Pier Siebesma, Claudia Christine Stephan, Peter P Sullivan, Ludovic Touzé-Peiffer, Jessica Vial, Raphaela Vogel, Paquita Zuidema, Nicola Alexander, Lyndon Alves, Sophian Arixi, Hamish Asmath, Gholamhossein Bagheri, Katharina Baier, Adriana Bailey, Dariusz B. Baranowski, Alexandre Baron, Sébastien Barrau, Paul Alan Barrett, Frédéric Batier, Andreas Behrendt, Arne Bendinger, Florent Beucher, Sebastien Bigorre, Edmund Blades, Olivier Bock, Steven J Boeing, Pierre Bosser, Denis Bourras, Pascale Bouruet-Aubertot, Keith Bower, Pierre Branellec, Hubert Branger, Michal Brennek, Alan Brewer, Pierre-Etienne Brilouet, Björn Brügmann, Stefan Alexander Buehler, Elmo Burke, Ralph Burton, Radiance Calmer, Jean-Christophe Canonici, Xavier J. Carton, Gregory Cato, Jude Andre Charles, Patrick Chazette, Yanxu Chen, Michał T Chiliński, Tom Choularton, Patrick Y. Chuang, Shamal Clarke, Hugh Coe, Céline Cornet, Pierre Coutris, Fleur Couvreux, Susanne Crewell, Timothy Wallace Cronin, Zhiqiang Cui, Yannis Cuypers, Alton Daley, Gillian Mary Damerell, Thibaut Dauhut, Hartwig M Deneke, Jean-Philippe Desbios, Steffen Dörner, Sebastian Donner, Vincent Douet, Kyla Drushka, Marina Dütsch, André Ehrlich, Kerry Emanual, Alexandros Emmanouilidis, Jean-Claude Etienne, Sheryl Etienne-Leblanc, Ghislain Faure, Graham Feingold, Luca Ferrero, Andreas Fix, Cyrille Flamant, Piotr J. Flatau, Gregory R. Foltz, Linda Forster, Iulian Furtuna, Alan Gadian, Joseph Galewsky, Martin Gallagher, Peter Gallimore, Cassandra J. Gaston, Chelle Gentemann, Nicolas Geyskens, Andreas Giez, John Gollop, Isabelle Gouirand, Christophe Gourbeyre, Dörte de Graaf, Geiske E. de Groot, Robert Grosz, Johannes Güttler, Manuel Gutleben, Kashawn Hall, George Harris, Kevin Christopher Helfer, Dean Henze, Calvert Herbert, Bruna Holanda, Antonio Ibanez-Landeta, Janet M. Intrieri, Suneil Iyer, Fabrice Julien, Heike Kalesse, Jan Kazil, Alexander Kellman, Abiel T. Kidane, Ulrike Kirchner, Marcus Klingebiel, Mareike Körner, Leslie Ann Kremper, Jan Kretzschmar, Ovid Krüger, Wojciech Kumala, Armin Kurz, Pierre L'Hégaret, Matthieu Labaste, Tom A. Lachlan-Cope, Arlene Laing, Peter Landschützer, Theresa Lang, Diego Lange Vega, Ingo Lange, Clément Laplace, Gaute Lavik, Rémi LAXENAIRE, Caroline Le Bihan, Mason Leandro, Nathalie Lefèvre, Marius Lena, Donald Leschow, Qiang Li, Gary Lloyd, Sebastian Los, Niccolò Losi, Oscar Lovell, Christopher Luneau, Przemysław Makuch, Szymon P Malinowski, Gas- 
ton Manta, Eleni Marinou, Nicholas Marsden, Sebastien Masson, Nicolas Maury, Bernhard Mayer, Margarette Mayers-Als, Christophe Mazel, Wayne McGeary, James C. McWilliams, Mario Mech, Melina Mehlmann, Agostino Niyonkuru Meroni, Theresa Mieslinger, Andreas Minikin, Peter Minnett, Gregor Möller, Yanmichel Morfa Avalos, Caroline Muller, Ionela Musat, Anna Napoli, Almuth Neuberger, Christophe Noisel, David C Noone, Freja Nordsiek, Jakub L Nowak, Lothar Oswald, Douglas J. Parker, Carolyn Peck, Renaud Person, Miriam Philippi, Albert J. Plueddemann, Christopher Pöhlker, Veronika Pörtge, Ulrich Pöschl, Lawrence Pologne, Michal Posyniak, Marc Prange, Estefanía Quiñones Meléndez, Jule Radtke, Karim Ramage, Jens Reimann, Lionel Renault, Klaus Reus, Ashford Reyes, Joachim Ribbe, Maximilian Ringel, Markus Ritschel, Cesar Barbedo Rocha, Nicolas Rochetin, Johannes Röttenbacher, Callum Rollo, Haley Royer, Pauline Sadoulet, Leo Saffin, Sanola Sandiford, Irina Sandu, Michael Schäfer, Vera Schemann, Imke Schirmacher, Oliver Schlenczek, Jerome Schmidt, Marcel Schröder, Alfons Schwarzenboeck, Andrea Sealy, Christoph J Senff, Ilya Serikov, Samkeyat Shohan, Elizabeth Siddle, Alexander Smirnov, Florian Späth, Branden Spooner, M. Katharina Stolla, Wojciech Szkółka, Simon P. de Szoeke, Stéphane Tarot, Eleni Tetoni, Elizabeth J Thompson, Jim Thomson, Lorenzo Tomassini, Julien Totems, Alma Anna Ubele, Leonie Villiger, Jan von Arx, Thomas Wagner, Andi Walther, Benjamin G. M. Webber, Manfred Wendisch, Shanice Whitehall, Anton Wiltshire, Allison A. Wing, Martin Wirth, Jonathan Wiskandt, Kevin Wolf, Ludwig Worbes, Ethan Wright, Volker Wulfmeyer, Shanea Young, Chidong Zhang, Dongxiao Zhang, Florian Ziemen, Tobias Zinner, and Martin Zöger. November 2020. doi: 10.1002/essoar.10504890.1.

[63] G. I. Taylor. Proceedings of the Royal Society A: Mathematical, Physical and Engineering Sciences, 164(919):476-490, February 1938. doi: 10.1098/rspa. 1938.0032 .

[64] Cameron Tropea, Alexander L. Yarin, and John F. Foss, editors. Springer Handbook of Experimental Fluid Mechanics. Springer, 1 edition, 2007.

[65] Paul A. Vaillancourt and M. K. Yau. Bulletin of the American Meteorological Society, 81(2):285-298, February 2000. doi: 10.1175/1520-0477(2000) 081<0285:ropiac>2.3.co;2.

[66] Greg A. Voth, A. La Porta, Alice M. Crawford, Jim Alexander, and Eberhard Bodenschatz. Journal of Fluid Mechanics, 469:121-160, October 2002. doi: $10.1017 / \mathrm{s} 0022112002001842$. 
[67] John M. Wallace and Peter V. Hobbs. Atmospheric Science. Elsevier, 2006. doi: 10.1016/c2009-0-00034-8.

[68] Lian-Ping Wang, Anthony S. Wexler, and Yong Zhou. Physics of Fluids, 10(10):2647-2651, October 1998. doi: 10.1063/1.869777.

[69] G. E. Willis and J. W. Deardorff. Quarterly Journal of the Royal Meteorological Society, 102(434):817-822, October 1976. doi: 10.1002/qj.49710243411.

[70] J. C. Wyngaard and S. F. Clifford. Journal of the Atmospheric Sciences, 34 (6):922-929, June 1977. doi: 10.1175/1520-0469(1977)034<0922:thahts $>2$. 0. co; 2 . 


\section{Statement regarding chapter-based thesis}

G. Bertens, G. Bagheri, H. Xu, E. Bodenschatz, J. Moláček, In-situ cloud particle tracking experiment, submitted to Rev. Sci. Instrum.

Direct inclusion of this article as a chapter did not suit the narrative of this thesis. Instead, material from this article is used throughout the thesis, in particular in sections 33 3.3, 4.1 (incl. subsections), and D.1. Dr. Moláček contributed most of section 4.1 (incl. subsections); the rest is my work. I am the article's editor; the contributions by all other authors, including those by Dr. Moláček, have been edited by me as I saw fit. 


\section{Curriculum Vitae}

\section{Education}

2006-2011 Applied Physics BSc., Eindhoven University of Technology, with a minor in Computer Science. Dissertation: Fluctuation, Dissipation and Turbulence; experimental analysis of turbulent convection of flow perturbations.

2011 Information Security Technology, Eindhoven University of Technology, University of Twente, Radboud University Nijmegen. Completed 24 ECTS towards a Master's degree. Courses included: network security, cryptography, and biometrics.

2012-2015 Applied Physics MSc. Eindhoven University of Technology, with a specialisation in fluid mechanics. Dissertation: Droplets and Turbulence. Experimental analysis of turbulent dispersion of droplets; analysis and improvement of PIV data processing for measuring the turbulent dissipation rate.

\section{Publications}

- G. Bertens, D. van der Voort, H. Bocanegra-Evans, W. van de Water, Large-eddy estimate of the turbulent dissipation rate using PIV, Exp. in Fluids 56:89, April 2015.

- H. Bocanegra-Evans, N. Dam, D. van der Voort, G. Bertens, W. van de Water, Measuring droplet size distributions from overlapping interferometric particle images, Rev. Sci. Instrum. 86, 023709, February 2015.

- H. Bocanegra-Evans, N. Dam, G. Bertens, D. van der Voort, W. van de Water, Dispersion of droplet clouds in turbulence, Phys. Rev. Lett., 117, 164501, October 2016. 
- H. Bocanegra-Evans, N. Dam, G. Bertens, W. van de Water, Making droplets glow in turbulence, Phys. Rev. Fluids. 5, 044303, April 2020.

- G. Bertens, G. Bagheri, H. Xu, E. Bodenschatz, J. Moláček, In-situ cloud particle tracking experiment, submitted to Rev. Sci. Instrum.

\section{Conference talks}

- G. Bertens, G. Bagheri, H. Xu, E. Bodenschatz, J. Moláček, Results from the Zugspitze Experiment: an in-situ cloud-droplet particle-tracking experiment, 17th European Turbulenc Conference, Turin, September 2019.

- G. Bertens, G. Bagheri, H. Xu, E. Bodenschatz, J. Moláček, Results from the Zugspitze Experiment: an in-situ cloud-droplet particle-tracking experiment, Third Infinity Conference, Göttingen, February 2020. 